\title{
HIGH-PRECISION RADIOCARBON AGE CALIBRATION FOR TERRESTRIAL AND MARINE SAMPLES
}

\section{MINZE STUIVER, PAULA J. REIMER and THOMAS F. BRAZIUNAS}

Department of Geological Sciences and Quaternary Research Center, Box 351360, University of Washington, Seattle, Washington 98195, USA

ABSTRACT. Single-year and decadal radiocarbon tree-ring ages are tabulated and discussed in terms of ${ }^{14} \mathrm{C}$ age calibration. The single-year data form the basis of a detailed ${ }^{14} \mathrm{C}$ age calibration curve for the cal AD 1510-1954 interval ("cal" denotes calibrated). The Seattle decadal data set (back to $11,617 \mathrm{cal}$ BP, with $0 \mathrm{BP}=\mathrm{AD} 1950$ ) is a component of the integrated decadal INTCAL98 ${ }^{14} \mathrm{C}$ age curve (Stuiver et al. 1998). Atmospheric ${ }^{14} \mathrm{C}$ ages can be transformed into ${ }^{14} \mathrm{C}$ ages of the global ocean using a carbon reservoir model. INTCAL98 ${ }^{14} \mathrm{C}$ ages, used for these calculations, yield global ocean ${ }^{14} \mathrm{C}$ ages differing slightly from previously published ones (Stuiver and Braziunas 1993b). We include discussions of offsets, error multipliers, regional ${ }^{14} \mathrm{C}$ age differences and marine ${ }^{14} \mathrm{C}$ age response to oceanic and atmospheric forcing.

\section{INTRODUCTION}

Radiocarbon ages of dendrochronologically dated wood samples, mostly 10-yr segments, were previously reported for the interval $6000 \mathrm{cal} \mathrm{BC}-\mathrm{cal} \mathrm{AD} 1950$. These ${ }^{14} \mathrm{C}$ measurements have now been extended back to $9668 \mathrm{cal} \mathrm{BC}$. We also applied some minor corrections to a portion of the ${ }^{14} \mathrm{C}$ ages reported for decadal samples (Stuiver and Becker 1993), multiple-year samples (Stuiver and Reimer 1993) and single-year samples (Stuiver 1993; Stuiver and Braziunas 1993a). Our additional data (9668-6000 cal BC) are given here, together with corrected (when applicable) decadal and singleyear ${ }^{14} \mathrm{C}$ ages for the intervals $6000 \mathrm{cal} \mathrm{BC}-\mathrm{cal} \mathrm{AD} 1950$ and cal AD 1510-1954, respectively. The decadal data (Table 1, Appendix) altogether incorporate the 11,617-0 cal BP interval. Single-year data are given in Table 2 (Appendix) for the cal AD 1510-1954 interval.

As reported in the 1993 Calibration Issue (Stuiver, Long and Kra 1993), the measured ${ }^{14} \mathrm{C}$ activities of the samples dated between 1977 and 1987 were corrected for small amounts of radon (Stuiver and Becker 1993). The original ages, calculated without applying a radon correction, were reported in Stuiver and Kra (1986). Additional information, discussed in the next section, reduces the radon correction to one half the 1993 value. The $1993{ }^{14} \mathrm{C}$ age correction of $\sim 10$ to $20{ }^{14} \mathrm{C} \mathrm{yr}$ for samples measured between 1977 and 1987 evidently was too large. For the 1998 calculations we halved the original radon correction to $\sim 5$ to $10{ }^{14} \mathrm{C}$ yr. The latest "correction of the 1993 correction" is small and its influence is usually limited to $\sim 10{ }^{14} \mathrm{C} \mathrm{yr}$.

Adjustments of the German oak and pine chronologies have been included. Both chronologies were extensively reevaluated at the University of Stuttgart-Hohenheim (Spurk et al. 1998). Whereas the German oak series yields absolute cal $\mathrm{AD} / \mathrm{BC}$ dates (through a continuous count from the present to the past), the latest part of the pine series is ${ }^{14} \mathrm{C}$-matched with the earliest portion of the oak chronology. This yields a cal BP scale with a margin of error of about two decades (Kromer and Spurk 1998; Spurk et al. 1998).

The materials used here for the AD interval are mainly derived from U.S. Pacific Northwestern, Californian and Canadian trees. A few Northern German oak samples were used as well. The trees are described in Table 2 of Stuiver and Becker (1986).

For the $\mathrm{BC}$ portion a limited number of samples from the Irish oak chronology (Pilcher et al. 1984) were used near $500 \mathrm{cal}$ BC. California trees overlap with the oak series from Southern Germany between cal AD 45 and 145 cal BC. The Seattle German Main-Donau oak and German pine samples 
cover the intervals 7748 cal BC-cal AD 45 and 9668-8007 cal BC, respectively. Cellulose (Stuiver, Burk and Quay 1984) was isolated from all decadal wood samples older than 150 cal BC.

After studying ring thickness patterns of individual tree sections, the Hohenheim group rejected the earlier absolute dating (relative to the master chronology) of some of these sections (e.g., where beetle-induced damage was evident). Our previously measured ${ }^{14} \mathrm{C}$ ages of the rejected sections had to be withdrawn. Replacement wood will be used for new measurements, but in the meantime Seattle ${ }^{14} \mathrm{C}$ ages are missing for midpoints 7566-7498, 7876-7758, and 8827-8757 cal BC. An additional gap due to a 41-yr shift in the master chronology concerns the midpoints 5256-5215 cal BC. In some instances we did not receive wood (sections 6166-6053, 6386-6356, 7316-7206, 7996-7886, 90579027, 9258-9242 and 9358-9332 cal BC).

\section{OFFSETS, “ERROR MULTIPLIER” AND RADON CORRECTION}

The most recent Seattle (S), Belfast (B) and Heidelberg (H) results are reported in this issue. Belfast results, adjusted for the shifts in German oak chronology (McCormac, personal communication) are based on Pearson and Qua (1993) and Pearson, Becker and Qua (1993). Pretoria/Groningen (P/G) results were reported previously (Vogel and van der Plicht 1993). Average offsets between the ${ }^{14} \mathrm{C}$ ages of the different laboratories are relatively small for the complete date sets, with $\mathrm{S}-\mathrm{B}=-13 \pm$ $1 \mathrm{yr}(\mathrm{n}=866), \mathrm{S}-\mathrm{H}=-25 \pm 2 \mathrm{yr}(\mathrm{n}=230)$ and $\mathrm{S}-\mathrm{P} / \mathrm{G}=-17 \pm 2 \mathrm{yr}(\mathrm{n}=194)$. The \pm equals one standard deviation $(\sigma)$ and $\mathrm{n}$ is the number of comparisons. Offsets for millennia are given in Table 3.

TABLE 3. Average and millennia offsets (in ${ }^{14} \mathrm{C}$ yr) between Seattle (S), Belfast (B), Heidelberg $(\mathrm{H})$ and Pretoria/Groningen $(\mathrm{P} / \mathrm{G}) . \sigma_{1}$ is the predicted average standard deviation in ${ }^{14} \mathrm{C}$ age differences. The variance beyond $\sigma_{1}$ is represented by $\sigma_{\mathrm{E}}$ (see text). Comparisons are based on decadal samples (see Stuiver et al. 1998).

\begin{tabular}{|c|c|c|c|c|c|c|c|c|}
\hline \multirow[b]{2}{*}{ Age $\mathrm{AD} / \mathrm{BC}$} & \multicolumn{3}{|c|}{ Offsets } & \multirow{2}{*}{\multicolumn{2}{|c|}{$\begin{array}{cc}\sigma_{1} & \sigma_{\mathrm{E}} \\
\mathrm{S}-\mathrm{B}\end{array}$}} & \multirow{2}{*}{$\begin{array}{cc}\sigma_{1} & \sigma_{\mathrm{E}} \\
\mathrm{S}-\mathrm{H} \\
\end{array}$} & \multirow{2}{*}{\multicolumn{2}{|c|}{$\begin{array}{cc}\sigma_{1} & \sigma_{\mathrm{E}} \\
S-(\mathrm{P} / \mathrm{G}\end{array}$}} \\
\hline & S-B & $\mathrm{S}-\mathrm{H}$ & $\mathrm{S}-\mathrm{P} / \mathrm{G}$ & & & & & \\
\hline $10-9$ ka BC & & $-12 \pm 5$ & & & & $34 \quad 17$ & & \\
\hline $9-8 \mathrm{ka} \mathrm{BC}$ & & $-18 \pm 4$ & & & & $32 \quad 24$ & & \\
\hline $8-7 \mathrm{ka} \mathrm{BC}$ & $10 \pm 5$ & $-26 \pm 6$ & & 34 & 47 & $36 \quad 39$ & & \\
\hline $7-6 \mathrm{ka} \mathrm{BC}$ & $-17 \pm 3$ & $-34 \pm 5$ & & 31 & 31 & 3636 & & \\
\hline $6-5 \mathrm{ka} \mathrm{BC}$ & $-34 \pm 3$ & $-56 \pm 9$ & & 29 & 31 & 3556 & & \\
\hline $5-4$ ka BC & $-11 \pm 3$ & $-28 \pm 5$ & & 28 & 24 & 3141 & & \\
\hline $4-3 \mathrm{ka} \mathrm{BC}$ & $-17 \pm 3$ & & $-18 \pm 2$ & 27 & 17 & & 20 & 19 \\
\hline $3-2 \mathrm{ka} \mathrm{BC}$ & $-17 \pm 2$ & & $-16 \pm 3$ & 25 & 27 & & 23 & 13 \\
\hline $2-1 \mathrm{ka} B C$ & $-1 \pm 3$ & & $4 \pm 9$ & 26 & 21 & & 23 & 8 \\
\hline $1-0 \mathrm{ka} \mathrm{BC}$ & $-6 \pm 2$ & & & 25 & 13 & & & \\
\hline AD $0-1 \mathrm{ka}$ & $-12 \pm 3$ & & & 27 & 11 & & & \\
\hline AD $1-2 \mathrm{ka}$ & $-15 \pm 2$ & & & 19 & 15 & & & \\
\hline $10 \mathrm{ka}$ BC-AD $2 \mathrm{ka}$ & $-13 \pm 1 \mathrm{yr}$ & $-25 \pm 2 y r$ & $-17 \pm 2 y r$ & 27 & 24 & 3435 & 22 & 13 \\
\hline
\end{tabular}

For Seattle (S) we usually report a $\sigma$ derived from the near-Gaussian counting statistics of the accumulated number of counts for the sample and standards. Additional information on the $\sigma$ in the ${ }^{14} \mathrm{C}$ age is derived from the reproducibility of ${ }^{14} \mathrm{C}$ age determinations in the Seattle laboratory, and interlaboratory comparisons that provide information on the sum total of uncertainty tied to the processes of wood allocation, dendro-age determination, sample pretreatment, laboratory ${ }^{14} \mathrm{C}$ determination, regional ${ }^{14} \mathrm{C}$ differences and individual tree ${ }^{14} \mathrm{C}$ differences. 
The reported age error can be used to predict the statistical variance in ${ }^{14} \mathrm{C}$ age differences when results of two laboratories are available for samples with the same cal age. The ${ }^{14} \mathrm{C}$ age differences of samples of identical cal age yield an offset (the average of the differences) and a (scatter) standard deviation $\sigma_{2}$. The $\sigma_{2}$ is compared to the standard deviation $\sigma_{1}$ predicted from the ${ }^{14} \mathrm{C}$ age errors reported by both laboratories. The increase in variance (excess variance) $\sigma_{\mathrm{E}}$ is derived from $\sigma_{\mathrm{E}}{ }^{2}=$ $\sigma_{2}^{2}-\sigma_{1}^{2}$. The ratio $\sigma_{2} / \sigma_{1}$ yields the "error multiplier" $\mathrm{k}$ (Stuiver 1982).

The above statistical considerations are valid for comparisons of ${ }^{14} \mathrm{C}$ determinations of identical samples. However, the samples to be compared here are rarely fully identical in that the time over which the sample is averaged (e.g., 10-yr vs. 3-yr samples) differs. Furthermore, the differences in cal age (time-midpoints) of the samples is usually variable. Different selection criteria (e.g., should two samples be compared if one is a $20-\mathrm{yr}$ and the other a 3-yr sample, and the difference in midpoints is $10 \mathrm{yr}$ ?) yield different $\sigma_{\mathrm{E}}\left(\mathrm{and} \mathrm{k}\right.$ ) estimates. Given these uncertainties, the following $\sigma_{\mathrm{E}}$ calculations (based on decadal sample files; see the INTCAL98 calibration (Stuiver et al. 1998) for the construction of the decades) are "order of magnitude" only.

The comparison of the $S{ }^{14} \mathrm{C}$ ages to those of $\mathrm{B}, \mathrm{H}$ and $\mathrm{P} / \mathrm{G}$ yields the $\sigma_{1}$ and $\sigma_{\mathrm{E}}$ values given in Table 3. For $S-B(n=859)$ and $S-H(n=230)$ comparisons, the $\sigma_{E}$ and $\sigma_{1}$ are of the same order of magnitude; for $\mathrm{S}-\mathrm{P} / \mathrm{G}(\mathrm{n}=194)$, the $\sigma_{\mathrm{E}}$ is more like half $\sigma_{1}$. Expressed differently, average $\mathrm{k}$ values are in the 1.3-1.4 range. Other estimates yielded $\mathrm{k}=0.7(\mathrm{n}=44)$ when comparing $\mathrm{S}^{14} \mathrm{C}$ ages of singleyear Pacific Northwest wood with S determinations of single-year Kodiak Island wood (Stuiver and Braziunas 1998), and $\mathrm{k}=1.2$ from evaluating counting stability in the Seattle laboratory over 4 years (Stuiver and Becker 1993).

Previously we discussed in much detail a small radon correction that had to be applied to measurements made between 1977 and 1987 (Stuiver and Becker 1993). An average count-rate difference of $0.279 \pm 0.045$ counts per minute $(\mathrm{cpm})$ was used for this correction. Since 1987 we have remeasured many samples for which newly determined ${ }^{14} \mathrm{C}$ ages can be compared to $1977-1987$ ones. The enlarged data set suggests a smaller radon correction, with a count-rate difference of $0.051 \pm 0.023$ $\mathrm{cpm}$. The 1993 paper also reported first day $v$ s. fourth day count-rate differences that were compatible with a radon contribution of $0.276 \pm 0.016 \mathrm{cpm}$. When adding similar first day-fourth day baseline information for $1992-1996$, the $0.276 \pm 0.016 \mathrm{cpm}$ radon excess estimate is lowered to $0.213 \pm$ $0.016 \mathrm{cpm}$.

The radon corrections $(0.051$ and $0.213 \mathrm{cpm})$ suggested by the above calculations differ significantly (at the $5.8 \sigma$ level). There is no obvious explanation for the difference but both methods suggest a smaller radon correction. The adjusted average count-rate difference (unweighted) for the two comparisons is $0.132 \mathrm{cpm}$, or $48 \%$ of the 1993 value. For the calculation of the ${ }^{14} \mathrm{C}$ ages listed in Tables 1 and 2 we used counting rate corrections of individual counters that average $0.132 \mathrm{cpm}$ for samples measured between 1977 and 1987. This effectively halves the radon correction previously (Stuiver, Long and Kra 1993) applied to tree-ring ${ }^{14} \mathrm{C}$ determinations made in Seattle between 1977 and 1987.

Most of the cal age midpoints in Table 1 represent the midpoint of decadal (10 ring) wood samples. Occasional departures from 10-yr rings are noted in Table 1.

\section{REgIONAL OFFSETS}

Regional offsets, relative to Washington (W), were reported previously (Stuiver and Braziunas 1998). Trees grown in Alaska (A), Russia (R), Tasmania (T) and South Chile (C) were used (details 
can be found in Stuiver and Braziunas 1998). The reported offsets are A $-\mathrm{W}=14 \pm 3 \mathrm{yr}$ (AD 18841932), $\mathrm{R}-\mathrm{W}=-6 \pm 6 \mathrm{yr}(\mathrm{AD} 1545-1615)$ and $2 \pm 6 \mathrm{yr}(\mathrm{AD} 1615-1715), \mathrm{T}-\mathrm{W}=25 \pm 7 \mathrm{yr}$ (estimated for the 19th century) and $\mathrm{C}-\mathrm{W}=38 \pm 5 \mathrm{yr}$ ( $\mathrm{AD} 1670-1722)$ and $21 \pm 5 \mathrm{yr}$ (19th century). The 19th century "Southern Hemispheric" (Chile and Tasmania) offset is $23 \pm 4{ }^{14} \mathrm{C}$ yr (reported incorrectly in Stuiver and Braziunas 1998 as $23 \pm 9$ yr).

The above regional offsets, which are not necessarily constant with time, are for "natural" conditions. During the first half of the twentieth century the ${ }^{14} \mathrm{C}$ levels were modified by fossil fuel $\mathrm{CO}_{2}$ releases that depressed atmospheric ${ }^{14} \mathrm{C}$ levels to a greater extent in the Northern Hemisphere (Northern Hemispheric fossil fuel $\mathrm{CO}_{2}$ emissions are much larger than Southern Hemispheric ones). Whereas 19th century Chile/Tasmania ${ }^{14} \mathrm{C}$ ages are ca. $23{ }^{14} \mathrm{C}$ yr older than those of Washington, this offset is reduced during the first half of the 20th century. There is even a switch to younger Southern Hemispheric ages ca. AD 1940 (Stuiver and Braziunas 1998; McCormac et al. 1998a,b).

\section{LABORATORY OFFSETS IN PINE AND BRISTLECONE PINE DATA}

The measurements of two laboratories, Seattle and Heidelberg, are now available for German pine samples (both this issue). In Figure 1 we compare the $\mathrm{S}$ and $\mathrm{H}^{14} \mathrm{C}$ dates of the German pine chronology. The cal ages reflect the latest reevaluation by the University of Stuttgart-Hohenheim treering laboratory (Spurk et al. 1998). There is substantial agreement, with an S-H offset of $-16 \pm 3 \mathrm{yr}$ $(\mathrm{n}=101)$ and an error multiplier $\mathrm{k}=1.20$.

For the older samples, the German and Irish oak chronologies are of crucial importance. ${ }^{14} \mathrm{C}$ results of the independent bristlecone pine chronology (Linick et al. 1986), as established at Tucson, Arizona (A), cover the 6554-5350 cal BC interval. When comparing these data to Belfast and Heidelberg oak results (Pearson, Becker and Qua 1993; Stuiver et al. 1998; Kromer and Spurk 1998), the bristlecone pine ${ }^{14} \mathrm{C}$ ages are, respectively, $19 \pm 4(\mathrm{n}=75)$ and $17 \pm 8(\mathrm{n}=24) \mathrm{yr}$ older. When comparing Seattle (Stuiver and Reimer 1993) measured bristlecone pine ${ }^{14} \mathrm{C}$ ages (1998 radon corrected) to bristlecone pine measured in Arizona (Linick et al. 1986), the offset is $25 \pm 8 \mathrm{yr}(\mathrm{n}=15)$ toward older Arizona ages. These offsets, on the order of one or two decades, fall within the range expected from laboratory measuring errors, cal age differences in midpoint and tree-ring length of the wood samples, and nonidentical regional ${ }^{14} \mathrm{C}$.

The bristlecone pine ${ }^{14} \mathrm{C}$ age offset with Seattle oak ${ }^{14} \mathrm{C}$ ages (with minor offset corrections, see Stuiver et al. 1998) is a surprisingly large $48 \pm 3 \mathrm{yr}(\mathrm{n}=80)$. The reason for this "anomalous" result is, at present, unknown.

\section{SINGLE-YEAR AGE CALIBRATION}

In the 1993 calibration issue, and also in Stuiver and Braziunas 1993a, a set of single-year ${ }^{14} \mathrm{C}$ results was reported for wood from the Pacific Northwest (Washington State). The data in Table 2 and Figure 2 are based on these ${ }^{14} \mathrm{C}$ results with two modifications: 1) adjustment of the ${ }^{14} \mathrm{C}$ determinations made between 1977 and 1987 for the minor change in radon correction, as discussed previously, and 2) the incorporation of single-year results from a Kodiak Island, Alaska, Sitka spruce tree.

The Alaskan Sitka spruce $\left(58^{\circ} \mathrm{N}, 153^{\circ} \mathrm{W}\right)$ covers the cal $\mathrm{AD} 1884-1932$ interval. Its ${ }^{14} \mathrm{C}$ ages are, on average, $14 \pm 3{ }^{14} \mathrm{C}$ yr older than Washington State results (Stuiver and Braziunas 1998). To obtain a reduced standard deviation, the Alaskan and Washington ${ }^{14} \mathrm{C}$ data were averaged after normalizing on Washington State (by reducing the Alaskan results by 14 yr). As noted previously (Stuiver 1993), 


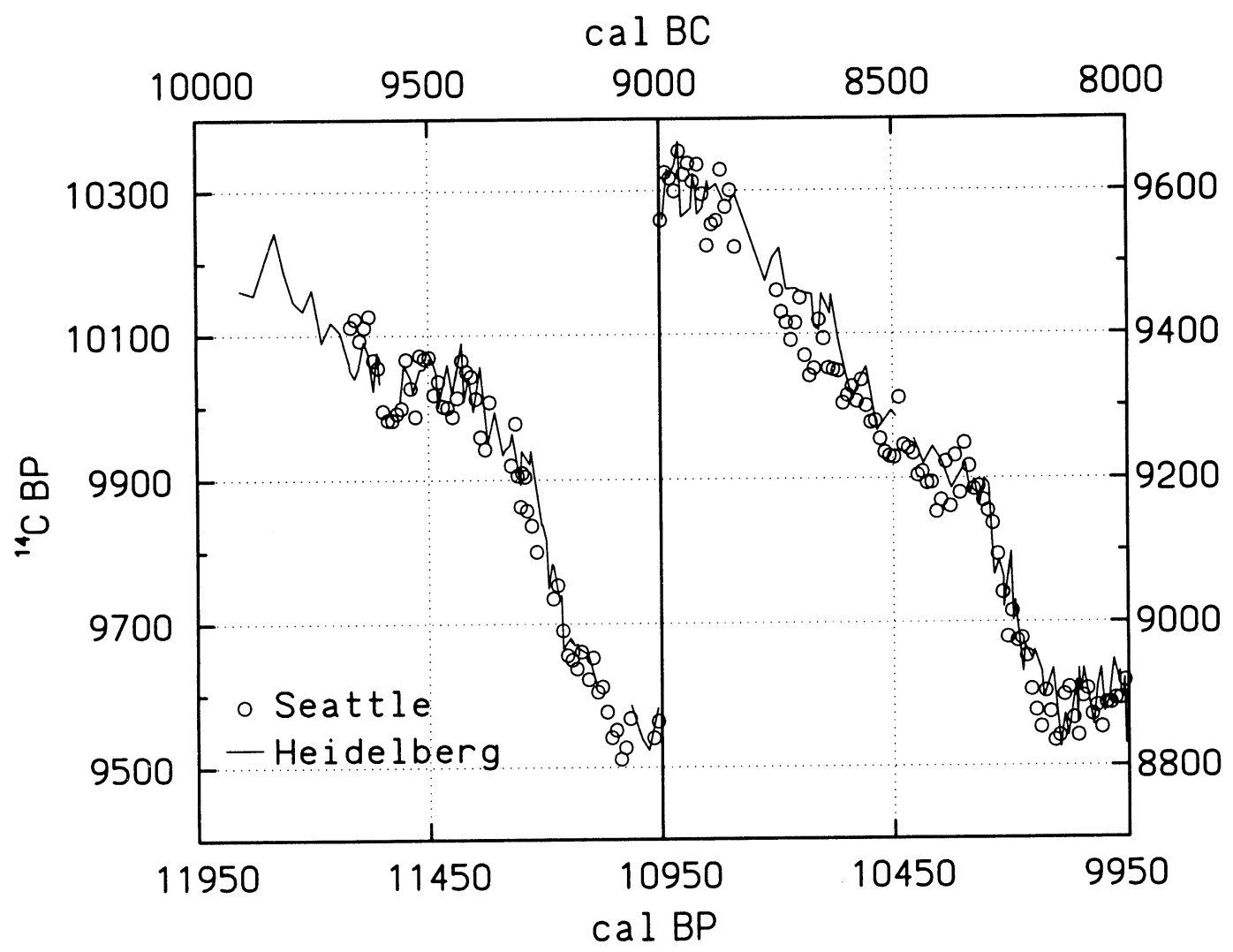

Fig. 1. A comparison of Heidelberg and Seattle German pine measurements. The solid line connects the Heidelberg points; the average standard deviation in a single measurement is 24 and $23{ }^{14} \mathrm{C}$ yr for, respectively, Heidelberg and Seattle.

the average standard deviation (for a 1.0 error multiplier) in the single-year calibration curve of 1993 was $12.8{ }^{14} \mathrm{C}$ yr. Adding Kodiak Island data reduces the average single-year standard deviation of the cal AD $1884-1932$ interval to $10.2{ }^{14} \mathrm{C}$ yr.

\section{MARINE ${ }^{14} \mathrm{C}$ AgE CALIBRATION}

With INTCAL 98 based on decadal averages, we no longer provide a separate (terrestrial) decadal Seattle curve. A model calculated marine curve, however, is still relevant. Extensive discussion of marine age calibration was presented in Stuiver, Pearson and Braziunas 1986, and Stuiver and Braziunas 1993b.

The 19th century reservoir age $\mathrm{R}_{\mathrm{g}}(\mathrm{t})(\mathrm{t}=$ time$)$ of the global ocean, relative to the atmosphere, is usually estimated at $400{ }^{14} \mathrm{C} \mathrm{yr}$ (its value prior to the industrial effect, or $c a$. $\mathrm{AD} 1850$ ). Marine reservoir age $\mathrm{R}_{\mathrm{g}}(\mathrm{t})$ varies over time as a result of geomagnetic and solar-related changes in ${ }^{14} \mathrm{C}$ production rates. $R_{g}(t)$ calculations suggests changes on the order of $\pm 100{ }^{14} \mathrm{C}$ yr for solar-mediated production rate change (Stuiver, Pearson and Braziunas 1986: Fig. 9A; Bard 1988).

The simple box diffusion global carbon cycle model employed here reproduces the expected history of global $\mathrm{R}_{\mathrm{g}}(\mathrm{t})$ in response to atmospheric ${ }^{14} \mathrm{C}$ production driven by solar and geomagnetic modulation of the ${ }^{14} \mathrm{C}$ production rate. To determine the variation in ${ }^{14} \mathrm{C}$ production rate required to produce 


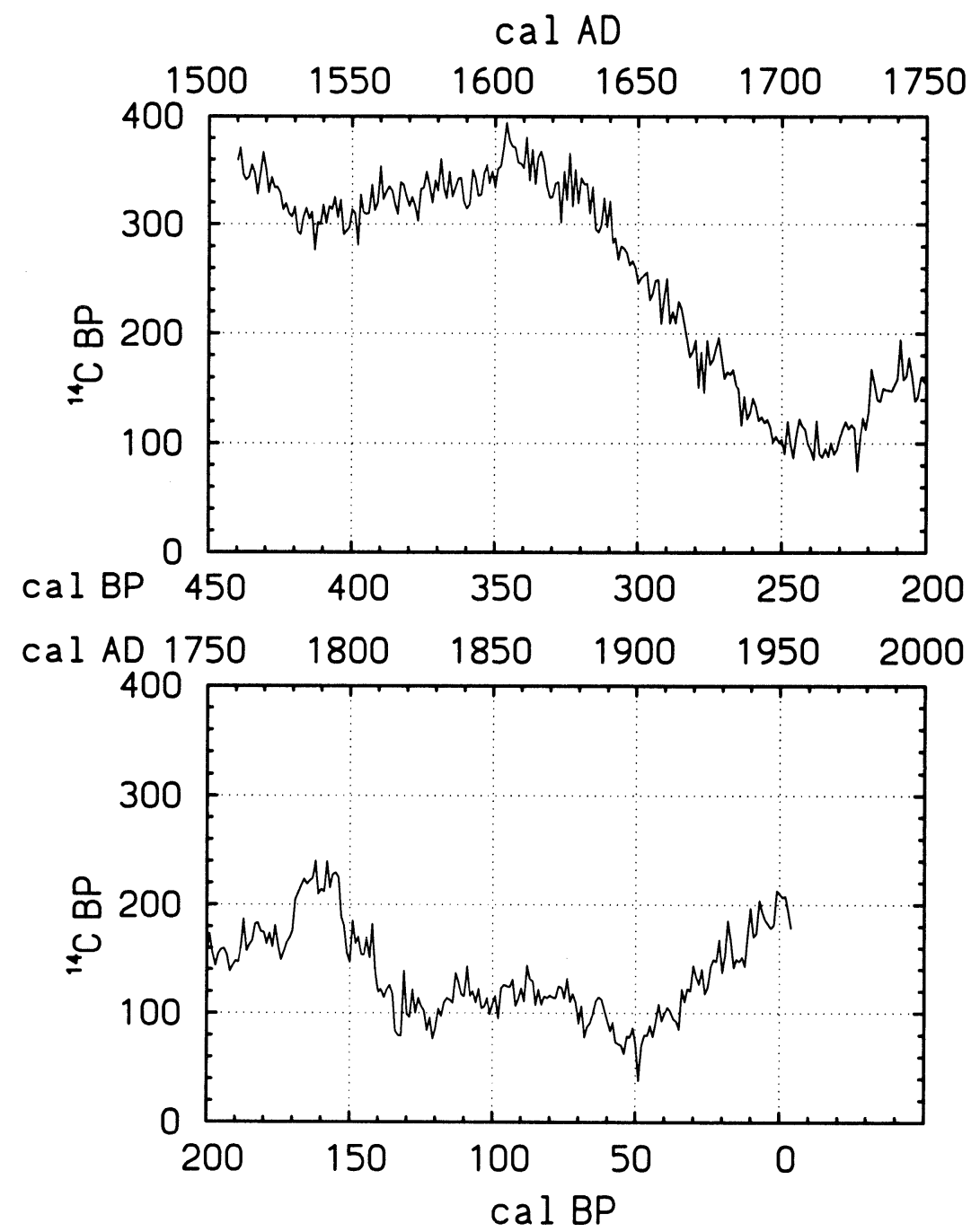

Fig. $2 .{ }^{14} \mathrm{C}$ age $v s$. cal age for single-year samples

the observed atmospheric record the model uses 1) the observed ${ }^{14} \mathrm{C}$ record from tree-rings; 2) a set of simple fixed parameters for ocean circulation, air-sea exchange, and atmosphere/terrestrial biosphere $\mathrm{CO}_{2}$ fluxes; 3 ) a reservoir age $\mathrm{R}_{\mathrm{g}}(\mathrm{AD} 1850)=400{ }^{14} \mathrm{C}$ yr; and 4$){ }^{14} \mathrm{C}$ information derived from corals to fix the initial ${ }^{14} \mathrm{C}$ level at the start of the Holocene. Model parameterization is discussed in Stuiver and Braziunas (1993b: 140).

Ocean circulation may also have affected the ${ }^{14} \mathrm{C}$ partitioning between atmosphere and global ocean, resulting in $R_{g}(t)$ change. Our $R_{g}(t)$ model response to oceanic, or production rate, forcing is depicted in Figure 3A. Starting with an approximately 200-yr-long plateau ( $9100-8900$ cal BC) in atmospheric ${ }^{14} \mathrm{C}$ ages (dashed curve, constructed from a 200 -yr moving average) we find substantial oceanic plateau smoothing ("surface ocean - 1" in Fig. 3A) for atmospheric forcing. However, when the ocean forces the atmosphere, both have similar plateau lengths ("surface ocean - 2" in Fig. 3A). Thus the presence or absence of ${ }^{14} \mathrm{C}$ age plateaus in marine sediment chronologies can be tied to the causative factors responsible for atmospheric ${ }^{14} \mathrm{C}$ change. 

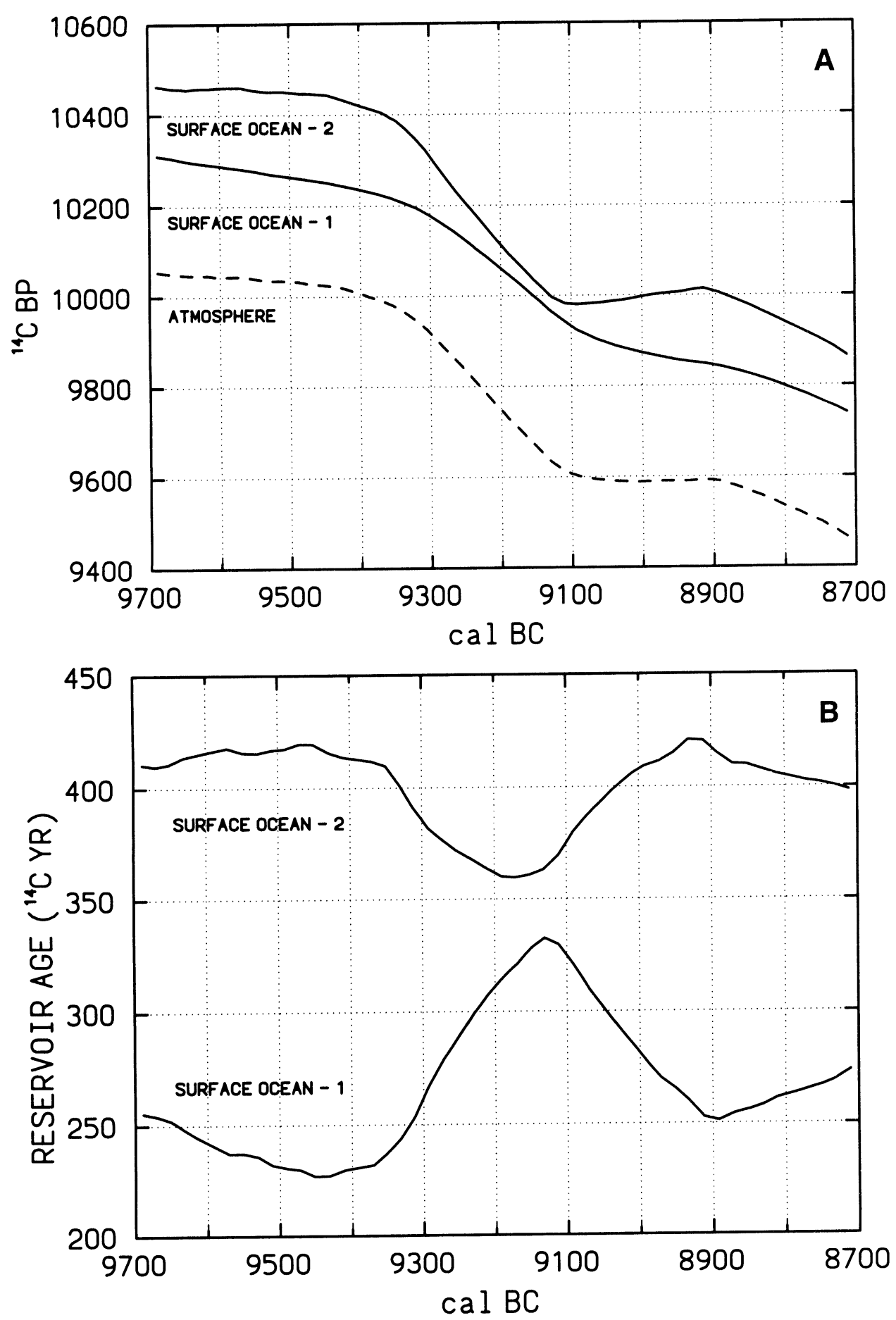

Fig. 3. A. Smoothed (200-yr moving average) ${ }^{14} \mathrm{C}$ age profiles for the atmosphere and surface ocean. Curve 1 was calculated from a carbon reservoir model assuming atmospheric ${ }^{14} \mathrm{C}$ production rate change to be responsible for the observed atmospheric ${ }^{14} \mathrm{C}$ change; curve 2 was calculated with ocean circulation change as the causal agent. B. Reservoir ages of the model ocean (mixed layer) for ${ }^{14} \mathrm{C}$ age plateaus generated by production rate change (curve 1) or oceanic circulation change (curve 2). 
Surface ocean reservoir ages differ substantially between scenarios based on production rate $v s$. oceanic circulation (Fig. 3B). Production-related atmospheric ${ }^{14} \mathrm{C}$ supply to the surface ocean results in concurrent fluxes to the deep ocean, whereas the atmosphere, when forced by the ocean, does not sustain such major losses to other reservoirs. As a result, the change in reservoir age is larger for the production rate scenario. Reservoir age perturbations also are opposite in sign because the ocean lags the atmosphere for the production-rate scenario, whereas the atmosphere lags the ocean for a postulated oceanic increase.

The possibility of oceanic-induced $R_{g}(t)$ change, on a century time scale, cannot be excluded. But nonhypothetical calculations of oceanic-induced $\operatorname{Rg}(\mathrm{t})$ change are not possible because detailed information on century time scale oceanic circulation change is lacking. The simple box-diffusion global carbon cycle calculations used to generate the solid line in Figure 4 assume, of necessity, that Holocene century-scale atmospheric ${ }^{14} \mathrm{C}$ variations are production rate related.

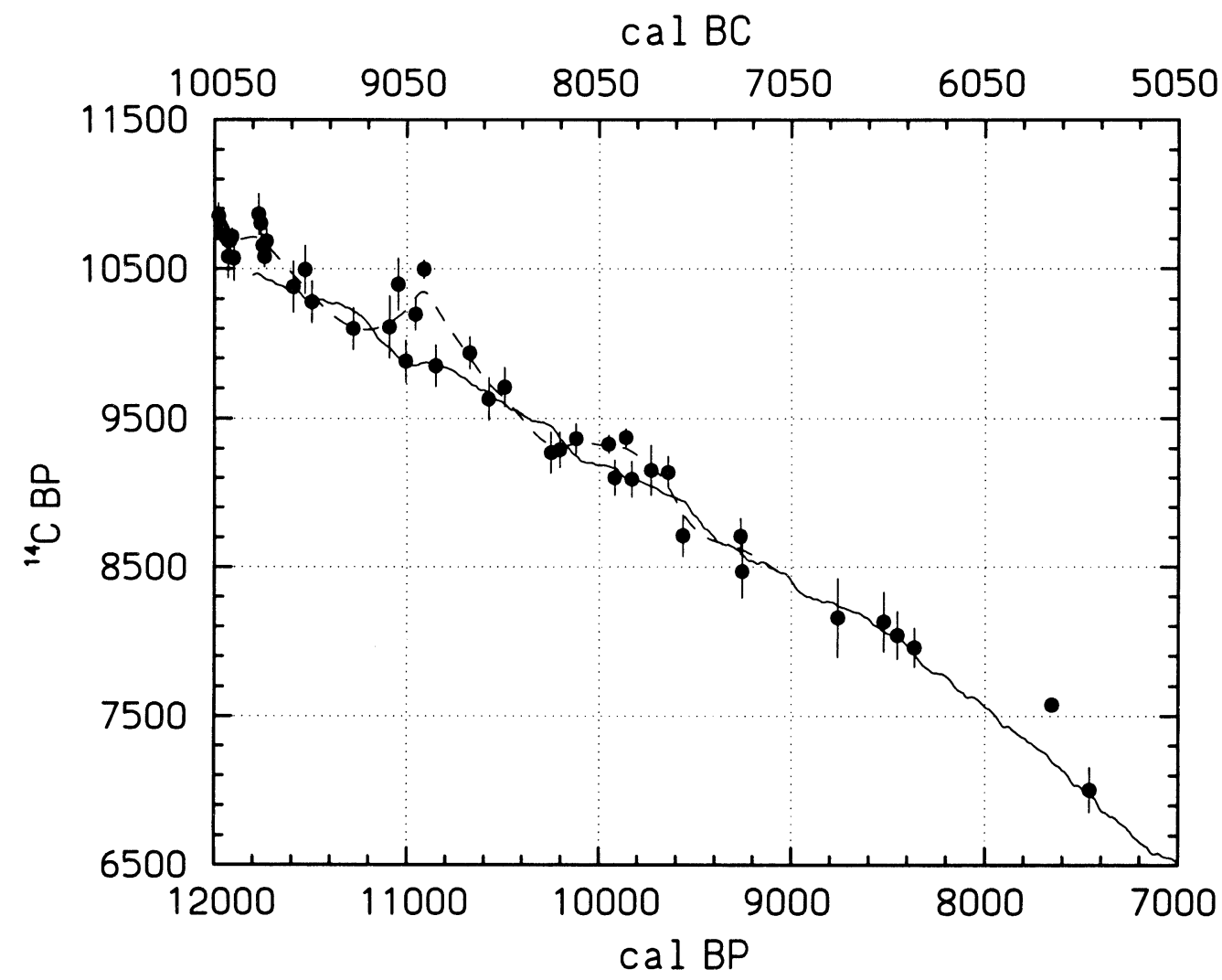

Fig. 4. A comparison of marine ${ }^{14} \mathrm{C}$ ages (solid line) derived from a carbon reservoir model (see text) and coral ${ }^{14} \mathrm{C}$ ages (Bard et al. 1998; Burr et al. 1998; Edwards et al. 1993; Stuiver et al. 1998: Fig. 2).

World ocean reservoir ages $R_{g}(t)$ increase (with a delayed response) when atmospheric ${ }^{14} \mathrm{C}$ increases and, conversely, are reduced when atmospheric ${ }^{14} \mathrm{C}$ levels drop. The reservoir ages $\mathrm{R}_{\mathrm{g}}(t)$ calculated for the world ocean are global averages only. Marine reservoir ages $(R(t, s)$, with $s=s p a c e)$ of the 19th century differ by up to $1000{ }^{14} \mathrm{C}$ yr from one oceanic region to another. The difference between regional reservoir age and the global average, $R(t, s)-R g(t)$, equals $\Delta R(s)$ (as defined in Stuiver and 
Braziunas 1993b). Implied in this definition is the notion that the time-dependent changes of the local environment parallel those of the global ocean, thus yielding a time-independent $\Delta R(s)$. Our approach has been to supply a model-derived $R_{g}(t)$, and estimate $\Delta R(s)$ from the measured reservoir ages of 19 th century shells $\left(\right.$ e.g., $\left.\Delta \mathrm{R}(\mathrm{s})=\mathrm{R}(\mathrm{AD} 1850, \mathrm{~s})-\mathrm{R}_{\mathrm{g}}(\mathrm{AD} 1850)\right)$. The measured ${ }^{14} \mathrm{C}$ age must be reduced by $\Delta R(s)$ when using the model-calculated marine calibration curves. We specifically note that the reservoir age $\mathrm{R}(\mathrm{t}, \mathrm{s})$ should not be subtracted but only $\Delta \mathrm{R}(\mathrm{s})(\Delta \mathrm{R}(\mathrm{s})=0$ when $\mathrm{R}(\mathrm{AD} 1850, \mathrm{~s})=400 \mathrm{yr})$. A short summary of regional $\Delta \mathrm{R}$ can be found in Stuiver and Braziunas (1993). Recent $\Delta \mathrm{R}$ determinations (partial list only) are those of Berkman and Forman (1996), Forman and Polyak (1997), Goodfriend and Flessa (1997), Heier-Nielsen et al. (1995), Higham and Hogg (1995), Ingram (1998), Ingram and Southon (1997), Kennett et al. (1997), Little (1993) and Southon, Rodman and True (1995).

Because the INTCAL98 tree-ring data for the 7000-0 cal BP interval are nearly identical to the data used previously, the 1993 marine calibration curves are still applicable (Stuiver and Braziunas 1993: Figs. 17A-N). Figure 4 compares the marine ${ }^{14} \mathrm{C}$ ages calculated from the INTCAL98 tree-ring record to those measured for INTCAL 98 corals (Bard et al. 1998; Burr et al. 1998; Edwards et al. 1993).

There is evidence for a marine ${ }^{14} \mathrm{C}$ reservoir deficiency change from 400 to $500{ }^{14} \mathrm{C}$ yr over the 12,000-10,000 cal BP interval (Stuiver et al. 1998: Fig. 2). This change, tied to ocean circulation change, is not simulated in the carbon reservoir model, where the ocean circulation parameters are fixed. This lack of ocean circulation change may have resulted in the slightly younger model-calculated ${ }^{14} \mathrm{C}$ ages of the $12,000-10,000$ cal BP interval (Fig. 4).

The number of coral data points between 9500 and $7000 \mathrm{cal}$ BP is limited, but the overall agreement is good for this interval. For the INTCAL 98 marine age calibration curve (see Stuiver et al. 1998) we used 1) a spline of coral and marine varve ${ }^{14} \mathrm{C}$ ages between 24,000 and $8800 \mathrm{cal} \mathrm{BP}$ and 2) a linear connection of ${ }^{14} \mathrm{C}$ ages derived from the tree-ring record via carbon reservoir modeling (8800-0 cal BP).

The latest 1998 version of the CALIB program (Stuiver and Reimer 1993) incorporates the singleyear data given here (and also the decadal INTCAL98 data set for marine and terrestrial environments). The data sets can be downloaded from the Quaternary Isotope Laboratory World Wide Web site $<$ http://depts.washington.edu/qil/>.

\section{ACKNOWLEDGMENTS}

The late Bernd Becker of the University of Stuttgart-Hohenheim, Germany, provided the samples from the German oak and pine chronology. M. Spurk and coworkers of the same university provided much needed information on the improved oak master chronology. B. Kromer and coworkers at the University of Heidelberg determined the pine-oak ${ }^{14} \mathrm{C}$ offset so that ring numbers of the floating pine chronology could be tied to cal BC dates. P. J. Wilkinson of the University of Washington provided crucial technical and analytical support. The National Science Foundation supported the ${ }^{14} \mathrm{C}$ investigations of the Quaternary Isotope Laboratory through grant ATM-9310121.

\section{REFERENCES}

Bard, E. 1988 Correction of accelerator mass spectrometry ${ }^{14} \mathrm{C}$ ages measured in planktonic foraminifera: $\mathrm{Pa}$ leoceanographic implications. Paleoceanography 3: $635-645$.
Bard, E., Arnold, M., Hamelin, B., Tisnerat-Laborde, N. and Cabioch, G. 1998 Radiocarbon calibration by means of mass spectrometric ${ }^{230} \mathrm{Th} /{ }^{234} \mathrm{U}$ and ${ }^{14} \mathrm{C}$ ages of corals: An updated database including samples 
from Barbados, Mururoa and Tahiti. Radiocarbon, this issue.

Berkman, P. A. and Forman, S. L. 1996 Pre-bomb radiocarbon and the reservoir correction for calcareous marine species in the Southern Ocean. Geophysical Research Letters 23: 363-366.

Burr, G. S., Beck, J. W., Taylor, F. W., Récy, J., Edwards, R. L. Cabioch, G., Corrège, T., Donahue, D. J. and O'Malley, J. M. 1998 A high-resolution radiocarbon calibration between 11,700 and 12,400 calendar years BP derived from ${ }^{230} \mathrm{Th}$ ages of corals from Espiritu Santo Island, Vanuatu. Radiocarbon, this issue.

Edwards, R. L., Beck, J. W., Burr, G. S., Donahue, D. J., Chappell, J. M. A., Bloom, A. L., Druffel, E. R. M. and Taylor, F. W. 1993 A large drop in atmospheric ${ }^{14} \mathrm{C} /$ ${ }^{12} \mathrm{C}$ and reduced melting in the Younger Dryas, documented with ${ }^{230}$ Th ages of corals. Science 260: $962-$ 968.

Forman, S. L. and Polyak, L. 1997 Radiocarbon content of pre-bomb marine mollusks and variations in the ${ }^{14} \mathrm{C}$ reservoir age for coastal areas of the Barents and Kara seas, Russia. Geophysical Research Letters 24: 885888.

Goodfriend, G. A. and Flessa, K. W. 1997 Radiocarbon reservoir ages in the Gulf of California: Roles of upwelling and flow from the Colorado River. Radiocarbon 39(2): 139-148.

Heier-Nielsen, S., Heinemeier, J., Nielsen, H. L. and Rud, N. 1995 Recent reservoir ages for Danish fjords and marine waters. Radiocarbon 37(3): 875-882.

Higham, T. F. G. and Hogg, A. G. 1995 Radiocarbon dating of prehistoric shell from New Zealand and calculation of the $\Delta \mathrm{R}$ value using fish otoliths. In Cook, G T., Harkness, D. D., Miller, B. F. and Scott, E. M., eds., Proceedings of the 15 th International ${ }^{14} \mathrm{C}$ Conference. Radiocarbon 37(2): 409-416.

Ingram, B. L. 1998 Differences in radiocarbon age between shell and charcoal from a Holocene shellmound in northern California. Quaternary Research 49: 102110.

Ingram, B. L. and Southon, J. R. 1997 Reservoir ages in eastern Pacific coastal and marine waters. Radiocarbon 38(3): 573-582.

Kennett, D. J., Ingram, B. L., Erlandson J. M. and Walker, P. 1997 Evidence for temporal fluctuations in marine radiocarbon reservoir ages in the Santa Barbara Channel, Southern California. Journal of Archae ological Science 24: 1051-1059.

Kromer, B. and Spurk, M. 1998 Revision and tentative extension of the tree-ring based ${ }^{14} \mathrm{C}$ calibration, 9200 $11,855 \mathrm{cal} \mathrm{BP}$. Radiocarbon, this issue.

Linick, T. W., Long, A., Damon, P. E. and Ferguson, C. W. 1986 High-precision radiocarbon dating of bristlecone pine from 6554 to $5350 \mathrm{BC}$. In Stuiver, M. and Kra, R., eds., Calibration Issue. Radiocarbon 28(2B): 943-953.

Little, E. A. 1993 Radiocarbon age calibration at archae- ological sites of coastal Massachusetts and vicinity. Journal of Archaeological Science 20: 457-471.

McCormac, F. G., Hogg, A. G., Higham, T. F. G., LynchStieglitz, J., Broecker, W. S., Baillie, M. G. L., Palmer, J., Xiong, L., Pilcher, J. R., Brown, D. and Hoper S. T. 1998a Temporal variation in the interhemispheric ${ }^{14} \mathrm{C}$ offset. Geophysical Research Letters 25: 1321-1324.

McCormac, F. G., Hogg, A. G., Higham, T. F. G., Baillie, M. G. L., Palmer, J. G., Xiong, L., Pilcher, J. R., Brown, D. and Hoper, S. T. 1998b Variations of radiocarbon in tree rings: Southern Hemisphere offset preliminary results. Radiocarbon, this issue.

Pearson, G. W., Becker, B. and Qua, F. 1993 High-precision ${ }^{14} \mathrm{C}$ measurement of German and Irish oaks to show the natural ${ }^{14} \mathrm{C}$ variations from 7890 to $5000 \mathrm{BC}$. In Stuiver, M., Long, A. and Kra, R. S., eds., Calibration 1993. Radiocarbon 35(1): 93-104.

Pearson, G. W. and Qua, F. 1993 High-precision ${ }^{14} \mathrm{C}$ measurement of Irish oaks to show the natural ${ }^{14} \mathrm{C}$ variations from AD 1840-5000 BC: A correction. In Stuiver, M., Long, A. and Kra, R. S., eds., Calibration 1993. Radiocarbon 35(1): 105-123.

Pilcher, J. R., Baillie, M. G. L., Schmidt, B. and Becker, B. 1984 A 7,272-year tree-ring chronology for Western Europe. Nature 312: 150-152.

Southon, J. R., Rodman, A. O. and True, D. 1995 A comparison of marine and terrestrial radiocarbon ages from northern Chile. In Cook, G. T., Harkness, D. D., Miller, B. F. and Scott, E. M., eds., Proceedings of the 15th International ${ }^{14} \mathrm{C}$ Conference. Radiocarbon 37(2): 389-393.

Spurk, M., Friedrich, M., Hofmann, J., Remmele, S., Frenzel, B., Leuschner, H. H. and Kromer, B. 1998 Revisions and extension of the Hohenheim oak and pine chronologies: New evidence about the timing of the Younger Dryas / Preboreal transition. Radiocarbon, this issue.

Stuiver, M. 1982 A high-precision calibration of the AD radiocarbon time scale. Radiocarbon 24(1): 1-26.

Stuiver, M. 1993 A note on single-year calibration of the radiocarbon time scale, AD 1510-1954. In Stuiver, M., Long, A. and Kra, R. S., eds., Calibration 1993. Radiocarbon 35(1): 67-72.

Stuiver, M. and Becker, B. 1986 High-precision decadal calibration of the radiocarbon time scale, AD 19502500 BC. In Stuiver, M. and Kra, R., eds., Calibration Issue. Radiocarbon 28(2B): 863-910.

1993 High-precision decadal calibration of the radiocarbon time scale, AD 1950-6000 BC. In Stuiver, M., Long, A. and Kra, R. S., eds., Calibration 1993. Radiocarbon 35(1): 35-65.

Stuiver, M. and Braziunas, T. F. 1993a Sun, ocean, climate and atmospheric ${ }^{14} \mathrm{CO}_{2}$ : An evaluation of causal and spectral relationships. The Holocene 3: 289-305.

$1993 \mathrm{~b}$ Modeling atmospheric ${ }^{14} \mathrm{C}$ influences and ${ }^{14} \mathrm{C}$ ages of marine samples to $10,000 \mathrm{BC}$. In Stuiver, M., Long, A. and Kra, R. S., eds., Calibration 1993. 
Radiocarbon 35(1): 137-189.

Stuiver, M. and Braziunas, T. F. 1998 Anthropogenic and solar components of hemispheric ${ }^{14} \mathrm{C}$. Geophysical Research Letters 25: 329-332.

Stuiver, M., Burk, R. L. and Quay, P. D. $1984{ }^{13} \mathrm{C} /{ }^{12} \mathrm{C}$ ratios in tree rings and the transfer of biospheric carbon to the atmosphere. Journal of Geophysical Research 89: 11,731-11,748.

Stuiver, M. and Kra, R., eds. 1986 Calibration Issue. $R a$ diocarbon 28(2B): 805-1030.

Stuiver, M., Long, A. and Kra, R. S., eds., Calibration 1993. Radiocarbon 35(1): 1-244.

Stuiver, M., Pearson, G. W. and Braziunas, T. F. 1986 Radiocarbon age calibration of marine samples back to 9000 cal yr BP. In Stuiver, M. and Kra, R., eds., Calibration Issue. Radiocarbon 28(2B): 980-1021.
Stuiver, M. and Polach, H. A. 1977 Discussion: Reporting of ${ }^{14} \mathrm{C}$ data. Radiocarbon 19(3): 355-363.

Stuiver, M. and Reimer, P. J. 1993 Extended ${ }^{14} \mathrm{C}$ data base and revised CALIB $3.0{ }^{14} \mathrm{C}$ age calibration program. In Stuiver, M., Long, A. and Kra, R. S., eds., Calibration 1993. Radiocarbon 35(1): 215-230.

Stuiver, M., Reimer, P. J., Bard, E., Beck, J. W., Burr, G. S., Hughen, K. A., Kromer, B., McCormac, G., van der Plicht, J. and Spurk, M. 1998 INTCAL98 radiocarbon age calibration $24,000-0$ cal BP. Radiocarbon, this issue.

Vogel, J. C. and van der Plicht, J. 1993 Calibration curve for short-lived samples, 1900-3900 BC. In Stuiver, M., Long, A. and Kra, R. S., eds., Calibration 1993. Radiocarbon 35(1): 87-91.

\section{APPENDIX: TABLES 1 AND 2}

TABLE $1 .{ }^{14} \mathrm{C}$ age determinations made at the University of Washington Quaternary Isotope Lab (Seattle). The cal $\mathrm{AD} / \mathrm{BC}$ ages (or cal $\mathrm{BP}$ ) represent midpoints to the nearest year of wood sections (10 $\mathrm{yr}$ unless given in parentheses with the cal $\mathrm{AD} / \mathrm{BC}$ age). Overlapping decadal samples with midpoints less than $1 \mathrm{yr}$ apart were averaged. Single-year data were averaged with decadal data for the AD 1515-1935 interval. Single-year data only were used for the AD 1945 data point. Results of the few 20-yr samples were taken as two decadal samples $(*)$ with the same ${ }^{14} \mathrm{C}$ age and with the standard deviation in the age and $\Delta^{14} \mathrm{C}$ (defined in Stuiver and Polach 1977) increased by 1.4 times. No error multiplier has been included in the standard deviations.

TABLE 1. Decadal Measurements

\begin{tabular}{crrr}
\hline cal AD/BC & \multicolumn{1}{c}{$\Delta^{14} \mathrm{C}(\% \circ)$} & \multicolumn{1}{c}{${ }^{14} \mathrm{C}$ BP } & cal BP \\
\hline AD 1945 & $-22.8 \pm 0.5$ & $190 \pm 4$ & 5 \\
AD 1935 & $-17.4 \pm 0.5$ & $156 \pm 4$ & 15 \\
AD 1925 & $-14.0 \pm 0.4$ & $138 \pm 3$ & 25 \\
AD 1915 & $-9.2 \pm 0.3$ & $108 \pm 3$ & 35 \\
AD 1905 & $-4.2 \pm 0.4$ & $78 \pm 3$ & 45 \\
AD 1895 & $-2.8 \pm 0.4$ & $76 \pm 3$ & 55 \\
AD 1885 & $-4.5 \pm 0.4$ & $100 \pm 3$ & 65 \\
AD 1875 & $-5.2 \pm 0.4$ & $115 \pm 4$ & 75 \\
AD 1865 & $-4.2 \pm 0.5$ & $117 \pm 4$ & 85 \\
AD 1855 & $-3.5 \pm 0.5$ & $120 \pm 4$ & 95 \\
AD 1845 & $-1.6 \pm 0.5$ & $115 \pm 4$ & 105 \\
AD 1835 & $-0.5 \pm 0.4$ & $116 \pm 3$ & 115 \\
AD 1825 & $2.8 \pm 0.4$ & $99 \pm 3$ & 125 \\
AD 1815 & $3.2 \pm 0.5$ & $106 \pm 4$ & 135 \\
AD 1805 & $-2.3 \pm 0.5$ & $159 \pm 4$ & 145 \\
AD 1795 & $-6.2 \pm 0.5$ & $201 \pm 4$ & 155 \\
AD 1785 & $-6.9 \pm 0.6$ & $216 \pm 5$ & 165 \\
AD 1775 & $0.4 \pm 0.4$ & $167 \pm 4$ & 175 \\
AD 1765 & $1.4 \pm 0.5$ & $169 \pm 4$ & 185 \\
AD 1755 & $4.1 \pm 0.4$ & $156 \pm 3$ & 195 \\
AD 1745 & $4.6 \pm 0.5$ & $163 \pm 4$ & 205 \\
AD 1735 & $7.0 \pm 0.5$ & $153 \pm 4$ & 215 \\
AD 1725 & $13.2 \pm 0.4$ & $114 \pm 3$ & 225 \\
AD 1715 & $16.8 \pm 0.3$ & $95 \pm 3$ & 235 \\
AD 1705 & $16.7 \pm 0.3$ & $105 \pm 3$ & 245 \\
AD 1695 & $16.6 \pm 0.3$ & $115 \pm 2$ & 255 \\
AD 1685 & $14.9 \pm 0.4$ & $139 \pm 3$ & 265
\end{tabular}

TABLE 1. Decadal Measurements (Continued)

\begin{tabular}{crlr}
\hline cal AD/BC & \multicolumn{1}{c}{$\Delta^{14} \mathrm{C}(\%)$} & \multicolumn{1}{c}{${ }^{14} \mathrm{C}$ BP } & cal BP \\
\hline AD 1675 & $12.0 \pm 0.5$ & $172 \pm 4$ & 275 \\
AD 1665 & $8.5 \pm 0.5$ & $209 \pm 4$ & 285 \\
AD 1655 & $5.7 \pm 0.5$ & $241 \pm 4$ & 295 \\
AD 1645 & $3.5 \pm 0.5$ & $268 \pm 4$ & 305 \\
AD 1635 & $-0.2 \pm 0.5$ & $308 \pm 4$ & 315 \\
AD 1625 & $-2.2 \pm 0.5$ & $333 \pm 4$ & 325 \\
AD 1615 & $-3.2 \pm 0.5$ & $351 \pm 4$ & 335 \\
AD 1605 & $-3.7 \pm 0.5$ & $365 \pm 4$ & 345 \\
AD 1595 & $0.7 \pm 0.5$ & $340 \pm 4$ & 355 \\
AD 1585 & $2.7 \pm 0.5$ & $333 \pm 4$ & 365 \\
AD 1575 & $4.4 \pm 0.6$ & $329 \pm 5$ & 375 \\
AD 1565 & $6.0 \pm 0.5$ & $326 \pm 4$ & 385 \\
AD 1555 & $8.3 \pm 0.6$ & $319 \pm 5$ & 395 \\
AD 1545 & $10.6 \pm 0.5$ & $309 \pm 4$ & 405 \\
AD 1535 & $12.8 \pm 0.4$ & $301 \pm 3$ & 415 \\
AD 1525 & $11.3 \pm 0.5$ & $322 \pm 4$ & 425 \\
AD 1515 & $9.2 \pm 0.6$ & $349 \pm 5$ & 435 \\
AD 1505 & $10.5 \pm 1.0$ & $349 \pm 8$ & 445 \\
AD 1495 & $11.2 \pm 1.3$ & $353 \pm 10$ & 455 \\
AD 1485 & $9.0 \pm 1.7$ & $380 \pm 13$ & 465 \\
AD 1475 & $9.0 \pm 1.8$ & $390 \pm 14$ & 475 \\
AD 1465 & $9.6 \pm 1.8$ & $395 \pm 14$ & 485 \\
AD 1455 & $11.5 \pm 1.2$ & $390 \pm 10$ & 495 \\
AD 1445 & $9.2 \pm 1.5$ & $418 \pm 12$ & 505 \\
AD 1435 & $4.1 \pm 1.8$ & $468 \pm 14$ & 515 \\
AD 1425 & $1.4 \pm 1.7$ & $500 \pm 13$ & 525 \\
AD 1415 & $0.0 \pm 1.7$ & $520 \pm 14$ & 535 \\
& & &
\end{tabular}


TABLE 1. Decadal Measurements (Continued)

\begin{tabular}{|c|c|c|c|}
\hline cal $\mathrm{AD} / \mathrm{BC}$ & $\Delta^{14} \mathrm{C}(\% \circ)$ & ${ }^{14} \mathrm{C} \mathrm{BP}$ & cal BP \\
\hline AD 1405 & $-4.3 \pm 1.6$ & $565 \pm 13$ & 545 \\
\hline AD 1395 & $-4.3 \pm 1.8$ & $575 \pm 14$ & 555 \\
\hline AD 1385 & $-10.3 \pm 1.3$ & $633 \pm 11$ & 565 \\
\hline AD 1375 & $-11.6 \pm 1.4$ & $653 \pm 11$ & 575 \\
\hline AD 1365 & $-4.4 \pm 1.3$ & $604 \pm 11$ & 585 \\
\hline AD 1355 & $-4.8 \pm 1.4$ & $617 \pm 12$ & 595 \\
\hline AD 1345 & $0.8 \pm 1.4$ & $582 \pm 12$ & 605 \\
\hline AD 1335 & $4.7 \pm 1.2$ & $560 \pm 10$ & 615 \\
\hline AD 1325 & $-0.6 \pm 1.3$ & $613 \pm 11$ & 625 \\
\hline AD 1315 & $-0.8 \pm 1.2$ & $625 \pm 10$ & 635 \\
\hline AD 1305 & $0.0 \pm 1.0$ & $627 \pm 8$ & 645 \\
\hline AD 1295 & $-3.0 \pm 1.8$ & $661 \pm 15$ & 655 \\
\hline AD 1285 & $-8.2 \pm 1.7$ & $713 \pm 14$ & 665 \\
\hline AD 1275 & $-13.7 \pm 1.8$ & $767 \pm 15$ & 675 \\
\hline AD 1265 & $-11.0 \pm 1.8$ & $755 \pm 15$ & 685 \\
\hline AD 1255 & $-16.7 \pm 1.7$ & $811 \pm 14$ & 695 \\
\hline AD 1245 & $-14.6 \pm 1.8$ & $803 \pm 14$ & 705 \\
\hline AD 1235 & $-13.4 \pm 1.8$ & $804 \pm 14$ & 715 \\
\hline AD 1225 & $-11.1 \pm 1.2$ & $795 \pm 10$ & 725 \\
\hline AD 1215 & $-14.5 \pm 1.7$ & $832 \pm 14$ & 735 \\
\hline AD 1205 & $-17.6 \pm 1.7$ & $867 \pm 14$ & 745 \\
\hline AD 1195 & $-15.2 \pm 1.7$ & $857 \pm 14$ & 755 \\
\hline AD 1185 & $-17.7 \pm 1.6$ & $887 \pm 13$ & 765 \\
\hline AD 1175 & $-15.7 \pm 1.7$ & $881 \pm 14$ & 775 \\
\hline AD 1165 & $-11.6 \pm 1.2$ & $857 \pm 10$ & 785 \\
\hline AD 1155 & $-16.5 \pm 1.8$ & $907 \pm 15$ & 795 \\
\hline AD 1145 & $-22.7 \pm 1.2$ & $967 \pm 10$ & 805 \\
\hline AD 1136 & $-12.9 \pm 1.8$ & $896 \pm 15$ & 814 \\
\hline AD 1135 & $-14.7 \pm 2.0$ & $911 \pm 17$ & 815 \\
\hline AD 1125 & $-13.9 \pm 1.8$ & $915 \pm 15$ & 825 \\
\hline AD 1116 & $-17.4 \pm 1.8$ & $952 \pm 15$ & 834 \\
\hline AD 1115 & $-15.5 \pm 1.9$ & $937 \pm 16$ & 835 \\
\hline AD 1106 & $-15.1 \pm 1.7$ & $943 \pm 14$ & 844 \\
\hline AD 1105 & $-15.8 \pm 1.7$ & $49 \pm 14$ & 845 \\
\hline AD 1096 & $-13.8 \pm 1.9$ & $942 \pm 15$ & 854 \\
\hline AD 1095 & $-13.4 \pm 1.8$ & $940 \pm 15$ & 855 \\
\hline AD 1086 & $-9.9 \pm 1.8$ & $920 \pm 14$ & 864 \\
\hline $\mathrm{AD} 1085$ & $-9.2 \pm 1.1$ & $916 \pm 9$ & 865 \\
\hline AD 1076 & $-7.0 \pm 1.1$ & $07 \pm 9$ & 874 \\
\hline AD 1075 & $-5.7 \pm 1.8$ & $897 \pm 15$ & 875 \\
\hline AD 1066 & $-8.4 \pm 1.4$ & $928 \pm 11$ & 884 \\
\hline AD 1065 & $-6.7 \pm 1.7$ & $915 \pm 14$ & 885 \\
\hline AD 1056 & $-6.0 \pm 1.4$ & $918 \pm 12$ & 894 \\
\hline AD 1055 & $-8.2 \pm 1$ & $936 \pm 15$ & 895 \\
\hline AD 1046 & $-6.4 \pm 1.8$ & $931 \pm 15$ & 904 \\
\hline AD 1045 & $-4.7 \pm 1.8$ & $918 \pm 14$ & 905 \\
\hline AD 1036 & $-6.6 \pm 1.7$ & $942 \pm 14$ & 914 \\
\hline AD 1035 & $-8.2 \pm 1.8$ & $956 \pm 15$ & 915 \\
\hline AD 1025 & $-9.3 \pm 1.8$ & $974 \pm 14$ & 925 \\
\hline AD 1015 & $-14.8 \pm 1.8$ & $1029 \pm 15$ & 935 \\
\hline AD 1005 & $-10.3 \pm 1.8$ & $1002 \pm 15$ & 945 \\
\hline AD 995 & $-14.7 \pm 1.6$ & $1047 \pm 13$ & 955 \\
\hline AD 985 & $-15.8 \pm 1.6$ & $1066 \pm 13$ & 965 \\
\hline AD 975 & $-21.5 \pm 1.8$ & $1123 \pm 15$ & 975 \\
\hline 965 & $-19.5 \pm 1.8$ & $1116 \pm 15$ & 985 \\
\hline 955 & $-18.9 \pm 1.9$ & $1121 \pm 16$ & 995 \\
\hline 945 & $-17.5 \pm 1.9$ & $1119 \pm 15$ & 1005 \\
\hline 935 & $-19.7 \pm 1.6$ & $1147 \pm 13$ & 1015 \\
\hline 925 & $-18.1 \pm 1.5$ & $1143 \pm 12$ & 1025 \\
\hline & & & \\
\hline
\end{tabular}

TABLE 1. Decadal Measurements (Continued)

\begin{tabular}{|c|c|c|c|c|}
\hline \multicolumn{2}{|c|}{$\mathrm{cal} \mathrm{AD} / \mathrm{BC}$} & $\Delta^{14} \mathrm{C}(\% \circ)$ & ${ }^{14} \mathrm{C}$ BP & cal BP \\
\hline $\mathrm{AD}$ & 905 & $-9.4 \pm 1.3$ & $1091 \pm 11$ & 1045 \\
\hline $\mathrm{AD}$ & 895 & $-14.5 \pm 1.5$ & $1143 \pm 13$ & 1055 \\
\hline $\mathrm{AD}$ & 885 & $-16.3 \pm 1.6$ & $1167 \pm 13$ & 1065 \\
\hline $\mathrm{AD}$ & 875 & $-17.3 \pm 1.4$ & $1186 \pm 12$ & 1075 \\
\hline $\mathrm{AD}$ & 865 & $-17.1 \pm 1.5$ & $1194 \pm 12$ & 1085 \\
\hline$A D$ & 855 & $-16.9 \pm 1.1$ & $1201 \pm 10$ & 1095 \\
\hline$A D$ & 845 & $-15.5 \pm 1.4$ & $1200 \pm 12$ & 1105 \\
\hline $\mathrm{AD}$ & 835 & $-12.2 \pm 1.4$ & $1183 \pm 12$ & 1115 \\
\hline$A D$ & 825 & $-12.8 \pm 1.4$ & $1197 \pm 12$ & 1125 \\
\hline$A D$ & 815 & $-10.6 \pm 1.5$ & $1189 \pm 12$ & 1135 \\
\hline$A D$ & 805 & $-10.3 \pm 1.6$ & $1197 \pm 13$ & 1145 \\
\hline $\mathrm{AD}$ & 795 & $-11.2 \pm 0.9$ & $1213 \pm 8$ & 1155 \\
\hline $\mathrm{AD}$ & 785 & $-4.4 \pm 1.2$ & $1168 \pm 10$ & 1165 \\
\hline $\mathrm{AD}$ & 775 & $-12.9 \pm 1.3$ & $1247 \pm 11$ & 1175 \\
\hline $\mathrm{AD}$ & 765 & $-16.0 \pm 1.3$ & $1282 \pm 10$ & 1185 \\
\hline $\mathrm{AD}$ & 755 & $-16.5 \pm 1.0$ & $1296 \pm 8$ & 1195 \\
\hline $\mathrm{AD}$ & 745 & $-11.4 \pm 1.3$ & $1263 \pm 11$ & 1205 \\
\hline $\mathrm{AD}$ & 735 & $-8.2 \pm 1.2$ & $1248 \pm 10$ & 1215 \\
\hline $\mathrm{AD}$ & 725 & $-7.8 \pm 1.6$ & $1254 \pm 13$ & 1225 \\
\hline $\mathrm{AD}$ & 715 & $-9.0 \pm 1.7$ & $1273 \pm 13$ & 1235 \\
\hline $\mathrm{AD}$ & 705 & $-10.4 \pm 1.8$ & $1294 \pm 15$ & 1245 \\
\hline $\mathrm{AD}$ & 695 & $-5.2 \pm 2.0$ & $1262 \pm 16$ & 1255 \\
\hline $\mathrm{AD}$ & 685 & $-12.5 \pm 2.2$ & $1331 \pm 18$ & 1265 \\
\hline $\mathrm{AD}$ & 675 & $-10.5 \pm 2.1$ & $1324 \pm 17$ & 1275 \\
\hline $\mathrm{AD}$ & 665 & $-11.5 \pm 2.1$ & $1342 \pm 17$ & 1285 \\
\hline $\mathrm{AD}$ & 655 & $-13.9 \pm 1.9$ & $1371 \pm 16$ & 1295 \\
\hline $\mathrm{AD}$ & 645 & $-15.9 \pm 2.1$ & $1397 \pm 17$ & 1305 \\
\hline $\mathrm{AD}$ & 635 & $-22.4 \pm 1.4$ & $1460 \pm 12$ & 1315 \\
\hline $\mathrm{AD}$ & 625 & $-19.2 \pm 1.9$ & $1444 \pm 16$ & 1325 \\
\hline $\mathrm{AD}$ & 615 & $-17.8 \pm 3.9$ & $1442 \pm 32$ & 1335 \\
\hline $\mathrm{AD}$ & 605 & $-17.8 \pm 1.8$ & $1452 \pm 15$ & 1345 \\
\hline $\mathrm{AD}$ & 595 & $-21.2 \pm 2.0$ & $1489 \pm 17$ & 1355 \\
\hline $\mathrm{AD}$ & 585 & $-18.3 \pm 1.9$ & $1475 \pm 16$ & 1365 \\
\hline $\mathrm{AD}$ & 575 & $-19.7 \pm 2.1$ & $1497 \pm 17$ & 1375 \\
\hline $\mathrm{AD}$ & 565 & $-17.8 \pm 2.0$ & $1491 \pm 16$ & 1385 \\
\hline $\mathrm{AD}$ & 555 & $-18.8 \pm 2.0$ & $1509 \pm 17$ & 1395 \\
\hline$A D$ & 545 & $-16.3 \pm 1.4$ & $1497 \pm 11$ & 1405 \\
\hline $\mathrm{AD}$ & 535 & $-22.6 \pm 2.0$ & $1559 \pm 16$ & 1415 \\
\hline $\mathrm{AD}$ & 525 & $-25.3 \pm 1.2$ & $1 \pm 10$ & 1425 \\
\hline $\mathrm{AD}$ & 515 & $-21.9 \pm 1.7$ & $1573 \pm 14$ & 1435 \\
\hline $\mathrm{AD}$ & 505 & $-21.5 \pm 1.8$ & $1580 \pm 15$ & 1445 \\
\hline $\mathrm{AD}$ & 495 & $-18.1 \pm 1.6$ & $1561 \pm 13$ & 1455 \\
\hline $\mathrm{AD}$ & 485 & $-16.9 \pm 2.0$ & $1561 \pm 17$ & 1465 \\
\hline $\mathrm{AD}$ & 475 & $-15.5 \pm 1.9$ & $1560 \pm 15$ & 1475 \\
\hline $\mathrm{AD}$ & 465 & $-16.6 \pm 2.0$ & $1578 \pm 17$ & 1485 \\
\hline $\mathrm{AD}$ & 455 & $-15.6 \pm 2.0$ & $1580 \pm 17$ & 1495 \\
\hline $\mathrm{AD}$ & 445 & $-11.5 \pm 2.0$ & $1556 \pm 16$ & 1505 \\
\hline $\mathrm{AD}$ & 435 & $-10.8 \pm 2.0$ & $1560 \pm 16$ & 1515 \\
\hline $\mathrm{AD}$ & 425 & $-16.8 \pm 1.5$ & $1618 \pm 13$ & 1525 \\
\hline $\mathrm{AD}$ & 415 & $-18.1 \pm 2.0$ & $1639 \pm 16$ & 1535 \\
\hline $\mathrm{AD}$ & 405 & $-20.2 \pm 1.8$ & $1666 \pm 15$ & 1545 \\
\hline $\mathrm{AD}$ & 395 & $-19.0 \pm 1.5$ & $1666 \pm 13$ & 1555 \\
\hline $\mathrm{AD}$ & 385 & $-19.7 \pm 1.4$ & $1 \pm 11$ & 1565 \\
\hline $\mathrm{AD}$ & 375 & $-22.0 \pm 1.1$ & $1710 \pm 9$ & 1575 \\
\hline $\mathrm{AD}$ & 365 & $-16.5 \pm 1.8$ & $1675 \pm 15$ & 1585 \\
\hline $\mathrm{AD}$ & 355 & $-18.4 \pm 2.0$ & $1699 \pm 17$ & 1595 \\
\hline $\mathrm{AD}$ & 345 & $-17.0 \pm 1.8$ & & 1605 \\
\hline $\mathrm{AD}$ & 335 & $-19.6 \pm 2.0$ & $1729 \pm 16$ & 1615 \\
\hline $\mathrm{AD}$ & 325 & $-17.6 \pm 2.0$ & $1723 \pm 17$ & 1625 \\
\hline $\mathrm{AD}$ & 315 & $-22.9 \pm 2.0$ & $1775 \pm 16$ & \\
\hline
\end{tabular}


TABLE 1. Decadal Measurements (Continued)

\begin{tabular}{|c|c|c|c|c|}
\hline \multicolumn{2}{|c|}{$\mathrm{cal} \mathrm{AD/BC}$} & $\Delta^{14} \mathrm{C}(\% o)$ & ${ }^{14} \mathrm{C} B P$ & cal BP \\
\hline $\mathrm{AD}$ & 305 & $-21.4 \pm 1.9$ & $1773 \pm 16$ & 1645 \\
\hline $\mathrm{AD}$ & 295 & $-14.5 \pm 1.9$ & $1726 \pm 15$ & 1655 \\
\hline $\mathrm{AD}$ & 285 & $-17.7 \pm 1.9$ & $1762 \pm 16$ & 1665 \\
\hline $\mathrm{AD}$ & 275 & $-10.5 \pm 1.9$ & $1713 \pm 15$ & 1675 \\
\hline $\mathrm{AD}$ & 265 & $-8.1 \pm 1.4$ & $1703 \pm 11$ & 1685 \\
\hline $\mathrm{AD}$ & 255 & $-15.3 \pm 1.1$ & $1773 \pm 9$ & 1695 \\
\hline $\mathrm{AD}$ & 245 & $-14.5 \pm 1.0$ & $1774 \pm$ & 1705 \\
\hline $\mathrm{AD}$ & 235 & $-18.0 \pm 1.4$ & $1813 \pm 12$ & 1715 \\
\hline $\mathrm{AD}$ & 225 & $-17.6 \pm 2.0$ & $1819 \pm 17$ & 1725 \\
\hline $\mathrm{AD}$ & 215 & $-18.1 \pm 2.0$ & $1834 \pm 17$ & 1735 \\
\hline $\mathrm{AD}$ & 205 & $-19.0 \pm 1.4$ & $1852 \pm 12$ & 1745 \\
\hline $\mathrm{AD}$ & 195 & $-14.1 \pm 2.0$ & $1820 \pm 17$ & 1755 \\
\hline $\mathrm{AD}$ & 185 & $-11.4 \pm 1.8$ & $1808 \pm 15$ & 1765 \\
\hline $\mathrm{AD}$ & 175 & $-13.0 \pm 2.0$ & $1831 \pm 16$ & 1775 \\
\hline $\mathrm{AD}$ & 165 & $-13.1 \pm 2.2$ & $1841 \pm 18$ & 1785 \\
\hline $\mathrm{AD}$ & 155 & $-11.9 \pm 1.9$ & $1841 \pm 15$ & 1795 \\
\hline $\mathrm{AD}$ & 145 & $-10.2 \pm 2.0$ & $1837 \pm 17$ & 1805 \\
\hline $\mathrm{AD}$ & 135 & $-8.6 \pm 1.3$ & $1833 \pm 11$ & 1815 \\
\hline $\mathrm{AD}$ & 125 & $-15.7 \pm 1.1$ & $1901 \pm 9$ & 1825 \\
\hline $\mathrm{AD}$ & 115 & $-14.6 \pm 2.2$ & $1902 \pm 18$ & 1835 \\
\hline $\mathrm{AD}$ & 105 & $-13.4 \pm 2.1$ & $1902 \pm 17$ & 1845 \\
\hline $\mathrm{AD}$ & 95 & $-8.9 \pm 1.2$ & $1874 \pm 10$ & 1855 \\
\hline $\mathrm{AD}$ & 85 & $-9.3 \pm 2.0$ & $1888 \pm 16$ & 1865 \\
\hline $\mathrm{AD}$ & 75 & $-11.4 \pm 1.4$ & $1915 \pm 11$ & 1875 \\
\hline $\mathrm{AD}$ & 65 & $-12.9 \pm 1.4$ & $1936 \pm 11$ & 1885 \\
\hline $\mathrm{AD}$ & 55 & $-13.4 \pm 1.2$ & $1951 \pm 9$ & 1895 \\
\hline $\mathrm{AD}$ & 45 & $-16.8 \pm 1.0$ & $1988 \pm 8$ & 1905 \\
\hline $\mathrm{AD}$ & 35 & $-9.8 \pm 1.5$ & $1940 \pm 13$ & 1915 \\
\hline $\mathrm{AD}$ & 25 & $-13.9 \pm 1.4$ & $1983 \pm 11$ & 1925 \\
\hline $\mathrm{AD}$ & 15 & $-14.6 \pm 1.1$ & $2000 \pm 9$ & 1935 \\
\hline $\mathrm{AD}$ & 5 & $-11.3 \pm 1.0$ & $1982 \pm 8$ & 1945 \\
\hline & $5 \mathrm{BC}$ & $-18.0 \pm 1.4$ & $2046 \pm 12$ & 1954 \\
\hline & $6 \mathrm{BC}$ & $-18.3 \pm 2.0$ & $2049 \pm 16$ & 1955 \\
\hline & $15 \mathrm{BC}$ & $-11.1 \pm 1.2$ & $1999 \pm 10$ & 1964 \\
\hline & $16 \mathrm{BC}$ & $-11.5 \pm 2.0$ & $2003 \pm 16$ & 1965 \\
\hline & $25 \mathrm{BC}$ & $-12.7 \pm 2.0$ & $2022 \pm 16$ & 1974 \\
\hline & $26 \mathrm{BC}$ & $-14.2 \pm 1.8$ & $2035 \pm 15$ & 1975 \\
\hline & $35 \mathrm{BC}$ & $-10.4 \pm 1.1$ & $2012 \pm 10$ & 1984 \\
\hline & $36 \mathrm{BC}$ & $-8.7 \pm 1.0$ & $2000 \pm 8$ & 1985 \\
\hline & $45 \mathrm{BC}$ & $-14.6 \pm 1.2$ & $2056 \pm 10$ & 1994 \\
\hline & $46 \mathrm{BC}$ & $-9.9 \pm 2.0$ & $2019 \pm 16$ & 1995 \\
\hline & $55 \mathrm{BC}$ & $-13.7 \pm 1.3$ & $2057 \pm 11$ & 2004 \\
\hline & $56 \mathrm{BC}$ & $-17.3 \pm 1.3$ & $2089 \pm 11$ & 2005 \\
\hline & $65 \mathrm{BC}$ & $-15.7 \pm 1.4$ & $35 \pm 12$ & 2014 \\
\hline & $66 \mathrm{BC}$ & \pm 1.8 & $2088 \pm 15$ & 2015 \\
\hline & $75 \mathrm{BC}$ & $-14.5 \pm 2.0$ & $2085 \pm 17$ & 2024 \\
\hline & $76 \mathrm{BC}$ & $-13.1 \pm 2.0$ & $2075 \pm 17$ & 2025 \\
\hline & $85 \mathrm{BC}$ & $-8.9 \pm 1.4$ & $2049 \pm 11$ & 2034 \\
\hline & $86 \mathrm{BC}$ & $-13.1 \pm 2.0$ & $2084 \pm 16$ & 203 \\
\hline & $95 \mathrm{BC}$ & $5 \pm 1.9$ & $2096 \pm 15$ & 2044 \\
\hline & $96 \mathrm{BC}$ & $-12.6 \pm 2.0$ & $2090 \pm 16$ & 2045 \\
\hline & $105 \mathrm{BC}$ & $-8.6 \pm 2.0$ & $2066 \pm 16$ & 2054 \\
\hline & $106 \mathrm{BC}$ & $-12.3 \pm 1.6$ & $2097 \pm 13$ & 2055 \\
\hline & $115 \mathrm{BC}$ & $-8.2 \pm 2.0$ & $2073 \pm 17$ & 2064 \\
\hline & $116 \mathrm{BC}$ & $-13.0 \pm 1.5$ & $2113 \pm 12$ & 2065 \\
\hline & $125 \mathrm{BC}$ & $-10.1 \pm 2.1$ & $2097 \pm 17$ & 2074 \\
\hline & $126 \mathrm{BC}$ & $-13.8 \pm 1.5$ & $2129 \pm 12$ & 2075 \\
\hline & & $-7.1 \pm 2.1$ & $2083 \pm 17$ & 2084 \\
\hline & $136 \mathrm{BC}$ & $-9.4 \pm 2.0$ & $2102 \pm 16$ & 2085 \\
\hline & $145 \mathrm{BC}$ & $-5.6 \pm 1.6$ & $2080 \pm 13$ & 2094 \\
\hline
\end{tabular}

TABLE 1. Decadal Measurements (Continued)

\begin{tabular}{|c|c|c|c|}
\hline cal $\mathrm{AD} / \mathrm{BC}$ & $\Delta^{14} \mathrm{C}(\% \circ)$ & ${ }^{14} \mathrm{C} \mathrm{BP}$ & cal BP \\
\hline $146 \mathrm{BC}$ & $-9.6 \pm 1.9$ & $2114 \pm 16$ & 2095 \\
\hline $156 \mathrm{BC}$ & $-5.4 \pm 2.1$ & $2090 \pm 17$ & 2105 \\
\hline $166 \mathrm{BC}$ & $-6.0 \pm 1.3$ & $2104 \pm 11$ & 2115 \\
\hline $176 \mathrm{BC}$ & $-11.8 \pm 2.1$ & $2161 \pm 17$ & 2125 \\
\hline $186 \mathrm{BC}$ & $-10.4 \pm 2.0$ & $2159 \pm 17$ & 2135 \\
\hline $196 \mathrm{BC}$ & $-5.4 \pm 1.4$ & $2128 \pm 11$ & 2145 \\
\hline $206 \mathrm{BC}$ & $-10.2 \pm 1.9$ & $2177 \pm 15$ & 2155 \\
\hline $216 \mathrm{BC}$ & $-15.4 \pm 1.1$ & $2229 \pm 9$ & 2165 \\
\hline $226 \mathrm{BC}$ & $-7.8 \pm 1.5$ & $2177 \pm 12$ & 2175 \\
\hline $236 \mathrm{BC}$ & $-14.7 \pm 1.2$ & $2243 \pm 9$ & 2185 \\
\hline $246 \mathrm{BC}$ & $-12.3 \pm 1.5$ & $2233 \pm 12$ & 2195 \\
\hline $256 \mathrm{BC}$ & $-9.3 \pm 2.0$ & $2218 \pm 16$ & 2205 \\
\hline $266 \mathrm{BC}$ & $-13.6 \pm 1.9$ & $2263 \pm 16$ & 2215 \\
\hline $276 \mathrm{BC}$ & $-7.7 \pm 1.4$ & $2225 \pm 11$ & 2225 \\
\hline $286 \mathrm{BC}$ & $-8.6 \pm 1.8$ & $2242 \pm 15$ & 2235 \\
\hline 296 BC & $-3.9 \pm 1.4$ & $2212 \pm 11$ & 2245 \\
\hline $306 \mathrm{BC}$ & $-0.1 \pm 1.9$ & $2192 \pm 16$ & 2255 \\
\hline $316 \mathrm{BC}$ & $0.2 \pm 1.9$ & $2200 \pm 16$ & 2265 \\
\hline $326 \mathrm{BC}$ & $5.2 \pm 1.4$ & $2169 \pm 11$ & 2275 \\
\hline $336 \mathrm{BC}$ & $7.3 \pm 1.9$ & $2162 \pm 16$ & 2285 \\
\hline $346 \mathrm{BC}$ & $6.9 \pm 1.4$ & $2176 \pm 11$ & 2295 \\
\hline $356 \mathrm{BC}$ & $1.4 \pm 1.4$ & $2229 \pm 11$ & 2305 \\
\hline $366 \mathrm{BC}$ & $-1.8 \pm 1.4$ & $2265 \pm 11$ & 2315 \\
\hline $376 \mathrm{BC}$ & $0.1 \pm 1.4$ & $2260 \pm 11$ & 2325 \\
\hline $386 \mathrm{BC}$ & $-1.0 \pm 2.0$ & $2278 \pm 16$ & 2335 \\
\hline 396 ВС & $-5.7 \pm 1.4$ & $2324 \pm 12$ & 2345 \\
\hline $406 \mathrm{BC}$ & $-10.8 \pm 1.1$ & $2377 \pm 9$ & 2355 \\
\hline $416 \mathrm{BC}$ & $-16.8 \pm 2.0$ & $2434 \pm 16$ & 2365 \\
\hline $426 \mathrm{BC}$ & $-13.6 \pm 2.1$ & $2418 \pm 17$ & 2375 \\
\hline $436 \mathrm{BC}$ & $-16.5 \pm 2.0$ & $2452 \pm 16$ & 2385 \\
\hline $446 \mathrm{BC}$ & $-15.7 \pm 3.3$ & $2455 \pm 27$ & 2395 \\
\hline $456 \mathrm{BC}$ & $-19.1 \pm 2.3$ & $2493 \pm 18$ & 2405 \\
\hline $466 \mathrm{BC}$ & $-11.8 \pm 2.5$ & $2443 \pm 20$ & 2415 \\
\hline $476 \mathrm{BC}$ & $-6.1 \pm 1.4$ & $2406 \pm 12$ & 2425 \\
\hline $486 \mathrm{BC}$ & $-8.5 \pm 2.0$ & $2435 \pm 17$ & 2435 \\
\hline $496 \mathrm{BC}$ & $-6.3 \pm 1.5$ & $2428 \pm 12$ & 2445 \\
\hline $506 \mathrm{BC}$ & $-5.7 \pm 2.0$ & $2432 \pm 17$ & 2455 \\
\hline $516 \mathrm{BC}$ & $-4.7 \pm 1.6$ & $2433 \pm 12$ & 2465 \\
\hline $526 \mathrm{BC}$ & $-8.6 \pm 1.7$ & $2475 \pm 13$ & 2475 \\
\hline $536 \mathrm{BC}$ & $-2.5 \pm 1.3$ & $2436 \pm 11$ & 2485 \\
\hline $546 \mathrm{BC}$ & $-6.7 \pm 1.5$ & $2479 \pm 12$ & 2495 \\
\hline $556 \mathrm{BC}$ & $-5.9 \pm 1.8$ & $2482 \pm 14$ & 2505 \\
\hline $566 \mathrm{BC}$ & $-5.8 \pm 2.3$ & $2491 \pm 18$ & 2515 \\
\hline $576 \mathrm{BC}$ & $-4.3 \pm 2.2$ & $2489 \pm 18$ & 2525 \\
\hline $586 \mathrm{BC}$ & $1.0 \pm 2.2$ & $2456 \pm 18$ & 2535 \\
\hline $596 \mathrm{BC}$ & $-4.9 \pm 1.7$ & $2513 \pm 14$ & 2545 \\
\hline $606 \mathrm{BC}$ & $-3.9 \pm 1.8$ & $2515 \pm 15$ & 2555 \\
\hline 607 BC & $-1.8 \pm 2.1$ & $2499 \pm 17$ & 2556 \\
\hline $616 \mathrm{BC}$ & $0.1 \pm 1.6$ & $2492 \pm 13$ & 2565 \\
\hline 617 BC & $1.6 \pm 1.5$ & $2482 \pm 12$ & 2566 \\
\hline $626 \mathrm{BC}$ & $-0.8 \pm 1.7$ & $2509 \pm 14$ & 2575 \\
\hline 627 BC* & $4.6 \pm 2.9$ & $2467 \pm 23$ & 2576 \\
\hline 636 BC & $2.9 \pm 2.1$ & $2489 \pm 17$ & 2585 \\
\hline 637 BC* $^{*}$ & $5.8 \pm 2.9$ & $2467 \pm 23$ & 2586 \\
\hline $646 \mathrm{BC}$ & $4.9 \pm 2.0$ & $2483 \pm 16$ & 2595 \\
\hline $647 \mathrm{BC}$ & $6.3 \pm 2.1$ & $2472 \pm 17$ & 2596 \\
\hline 656 ВС & $7.0 \pm 1.4$ & $2475 \pm 11$ & 2605 \\
\hline $657 \mathrm{BC}^{*}$ & $13.0 \pm 2.8$ & $2429 \pm 22$ & 2606 \\
\hline $667 \mathrm{BC}$ & $14.2 \pm 2.8$ & $2429 \pm 22$ & 2616 \\
\hline & & & \\
\hline
\end{tabular}


TABLE 1. Decadal Measurements (Continued)

\begin{tabular}{|c|c|c|c|}
\hline cal $\mathrm{AD} / \mathrm{BC}$ & $\Delta^{14} \mathrm{C}(\% o)$ & ${ }^{14} \mathrm{C}$ BP & cal BP \\
\hline $707 \mathrm{BC}$ & $15.5 \pm 3.4$ & $2458 \pm 27$ & 2656 \\
\hline $717 \mathrm{BC}$ & $15.9 \pm 3.4$ & $2464 \pm 27$ & 2666 \\
\hline 727 вс* $^{*}$ & $19.6 \pm 2.0$ & $2445 \pm 16$ & 2676 \\
\hline $737 \mathrm{BC}^{*}$ & $20.8 \pm 2.0$ & $2445 \pm 16$ & 2686 \\
\hline $747 \mathrm{BC}^{*}$ & $21.3 \pm 3.0$ & $2451 \pm 23$ & 2696 \\
\hline $757 \mathrm{BC}^{*}$ & $22.5 \pm 3.0$ & $2451 \pm 23$ & 2706 \\
\hline 767 вс* & $11.3 \pm 2.8$ & $2550 \pm 22$ & 2716 \\
\hline $777 \mathrm{BC}^{*}$ & $12.5 \pm 2.8$ & $2550 \pm 22$ & 2726 \\
\hline $787 \mathrm{BC}$ & $14.0 \pm 1.6$ & $2548 \pm 12$ & 2736 \\
\hline $797 \mathrm{BC}$ & $7.6 \pm 1.5$ & $2608 \pm 12$ & 2746 \\
\hline $807 \mathrm{BC}$ & $4.0 \pm 1.6$ & $2647 \pm 13$ & 2756 \\
\hline $817 \mathrm{BC}$ & $2.2 \pm 2.1$ & $2670 \pm 17$ & 2766 \\
\hline $827 \mathrm{BC}$ & $3.1 \pm 1.6$ & $2673 \pm 13$ & 2776 \\
\hline $837 \mathrm{BC}$ & $-3.6 \pm 1.6$ & $2737 \pm 13$ & 2786 \\
\hline $847 \mathrm{BC}$ & $-3.9 \pm 1.6$ & $2749 \pm 13$ & 2796 \\
\hline $857 \mathrm{BC}$ & $-2.6 \pm 2.1$ & $2748 \pm 17$ & 2806 \\
\hline $867 \mathrm{BC}$ & $-0.3 \pm 2.0$ & $2740 \pm 16$ & 2816 \\
\hline $877 \mathrm{BC}$ & $1.1 \pm 2.1$ & $2738 \pm 17$ & 2826 \\
\hline 887 вC & $6.0 \pm 2.1$ & $2708 \pm 17$ & 2836 \\
\hline 897 вС & $3.2 \pm 2.1$ & $2741 \pm 17$ & 2846 \\
\hline $907 \mathrm{BC}$ & $-0.3 \pm 2.0$ & $2778 \pm 16$ & 2856 \\
\hline $917 \mathrm{BC}$ & $0.6 \pm 2.1$ & $2781 \pm 17$ & 2866 \\
\hline $927 \mathrm{BC}$ & $0.5 \pm 2.1$ & $2791 \pm 17$ & 2876 \\
\hline $937 \mathrm{BC}$ & $0.2 \pm 1.6$ & $2804 \pm 13$ & 2886 \\
\hline $947 \mathrm{BC}$ & $-1.6 \pm 1.5$ & $2827 \pm 12$ & 2896 \\
\hline 957 вC & $0.1 \pm 1.7$ & $2824 \pm 13$ & 2906 \\
\hline $967 \mathrm{BC}$ & $9.2 \pm 2.1$ & $2761 \pm 17$ & 2916 \\
\hline $977 \mathrm{BC}$ & $0.0 \pm 1.5$ & $2844 \pm 12$ & 2926 \\
\hline $987 \mathrm{BC}$ & $2.5 \pm 2.0$ & $2834 \pm 16$ & 2936 \\
\hline 997 ВС & $5.6 \pm 2.0$ & $2818 \pm 16$ & 2946 \\
\hline $1007 \mathrm{BC}$ & $4.7 \pm 2.1$ & $2836 \pm 17$ & 2956 \\
\hline $1017 \mathrm{BC}$ & $1.4 \pm 2.0$ & $2871 \pm 16$ & 2966 \\
\hline $1027 \mathrm{BC}$ & $0.4 \pm 2.2$ & $2889 \pm 18$ & 2976 \\
\hline $1037 \mathrm{BC}$ & $2.8 \pm 2.1$ & $2880 \pm 17$ & 2986 \\
\hline $1047 \mathrm{BC}$ & $7.7 \pm 2.2$ & $2850 \pm 17$ & 2996 \\
\hline $1057 \mathrm{BC}$ & $-0.5 \pm 2.0$ & $2926 \pm 16$ & 3006 \\
\hline $1067 \mathrm{BC}$ & $7.0 \pm 2.3$ & $2876 \pm 18$ & 3016 \\
\hline $1077 \mathrm{BC}$ & $6.5 \pm 2.2$ & $2889 \pm 18$ & 3026 \\
\hline $1087 \mathrm{BC}$ & $4.5 \pm 2.2$ & $2915 \pm 18$ & 3036 \\
\hline $1097 \mathrm{BC}$ & $5.1 \pm 1.4$ & $2919 \pm 12$ & 3046 \\
\hline $1107 \mathrm{BC}$ & $7.6 \pm 1.5$ & $2909 \pm 12$ & 3056 \\
\hline $1117 \mathrm{BC}$ & $2.0 \pm 2.4$ & $2964 \pm 19$ & 3066 \\
\hline $1127 \mathrm{BC}$ & $10.1 \pm 2.1$ & $2909 \pm 17$ & 3076 \\
\hline $1137 \mathrm{BC}$ & $-0.3 \pm 1.4$ & $3002 \pm 11$ & 3086 \\
\hline $1147 \mathrm{BC}$ & $11.8 \pm 2.1$ & $2915 \pm 17$ & 3096 \\
\hline $1157 \mathrm{BC}$ & $8.9 \pm 2.2$ & $2947 \pm 17$ & 3106 \\
\hline $1167 \mathrm{BC}$ & $10.3 \pm 2.1$ & $2946 \pm 17$ & 3116 \\
\hline $1177 \mathrm{BC}$ & $11.2 \pm 2.6$ & $2949 \pm 21$ & 3126 \\
\hline $1187 \mathrm{BC}$ & $18.5 \pm 2.2$ & $2901 \pm 17$ & 3136 \\
\hline $1197 \mathrm{BC}$ & $6.6 \pm 2.0$ & $3005 \pm 16$ & 3146 \\
\hline $1207 \mathrm{BC}$ & $18.7 \pm 2.2$ & $2918 \pm 18$ & 3156 \\
\hline $1217 \mathrm{BC}$ & $10.4 \pm 1.2$ & $2992 \pm 9$ & 3166 \\
\hline $1227 \mathrm{BC}$ & $10.6 \pm 2.1$ & $3002 \pm 17$ & 3176 \\
\hline $1237 \mathrm{BC}$ & $16.5 \pm 2.3$ & $2965 \pm 18$ & 3186 \\
\hline $1247 \mathrm{BC}$ & $14.5 \pm 2.2$ & $2990 \pm 17$ & 3196 \\
\hline $1257 \mathrm{BC}$ & $16.4 \pm 1.7$ & $2985 \pm 14$ & 3206 \\
\hline $1267 \mathrm{BC}$ & $11.0 \pm 2.2$ & $3038 \pm 18$ & 3216 \\
\hline $1277 \mathrm{BC}$ & $13.0 \pm 2.1$ & $3032 \pm 17$ & 3226 \\
\hline $1287 \mathrm{BC}$ & $18.9 \pm 2.2$ & $2994 \pm 18$ & 3236 \\
\hline & $15.1+$ & $3035+17$ & \\
\hline
\end{tabular}

TABLE 1. Decadal Measurements (Continued)

\begin{tabular}{|c|c|c|c|}
\hline cal AD/BC & $\Delta^{14} \mathrm{C}(\% o)$ & ${ }^{14} \mathrm{C}$ BP & cal BP \\
\hline $1307 \mathrm{BC}$ & $17.3 \pm 2.3$ & $3027 \pm 18$ & 3256 \\
\hline $1317 \mathrm{BC}$ & $16.8 \pm 2.2$ & $3040 \pm 18$ & 3266 \\
\hline $1327 \mathrm{BC}$ & $10.2 \pm 1.9$ & $3102 \pm 15$ & 3276 \\
\hline $1337 \mathrm{BC}$ & $17.0 \pm 2.2$ & $3059 \pm 17$ & 3286 \\
\hline $1347 \mathrm{BC}$ & $20.2 \pm 2.2$ & $3043 \pm 18$ & 3296 \\
\hline $1357 \mathrm{BC}$ & $17.8 \pm 2.2$ & $3071 \pm 17$ & 3306 \\
\hline $1367 \mathrm{BC}$ & $24.2 \pm 2.1$ & $3030 \pm 16$ & 3316 \\
\hline $1377 \mathrm{BC}$ & $18.3 \pm 2.0$ & $3087 \pm 16$ & 3326 \\
\hline $1387 \mathrm{BC}$ & $19.0 \pm 2.1$ & $3091 \pm 16$ & 3336 \\
\hline $1397 \mathrm{BC}$ & $16.9 \pm 1.5$ & $3117 \pm 12$ & 3346 \\
\hline $1407 \mathrm{BC}$ & $20.4 \pm 2.0$ & $3099 \pm 16$ & 3356 \\
\hline $1417 \mathrm{BC}$ & $12.9 \pm 2.0$ & $3168 \pm 16$ & 3366 \\
\hline $1427 \mathrm{BC}$ & $20.4 \pm 2.2$ & $3118 \pm 17$ & 3376 \\
\hline 1437 ВC & $14.3 \pm 1.5$ & $3177 \pm 12$ & 3386 \\
\hline $1447 \mathrm{BC}$ & $15.6 \pm 1.5$ & $3177 \pm 12$ & 3396 \\
\hline $1457 \mathrm{BC}$ & $13.8 \pm 1.4$ & $3203 \pm 11$ & 3406 \\
\hline $1467 \mathrm{BC}$ & $12.5 \pm 1.5$ & $3222 \pm 12$ & 3416 \\
\hline $1477 \mathrm{BC}$ & $14.6 \pm 1.5$ & $3213 \pm 12$ & 3426 \\
\hline $1487 \mathrm{BC}$ & $19.4 \pm 1.7$ & $3185 \pm 13$ & 3436 \\
\hline 1497 вС & $14.8 \pm 2.3$ & $3231 \pm 18$ & 3446 \\
\hline $1507 \mathrm{BC}$ & $13.9 \pm 2.0$ & $3248 \pm 16$ & 3456 \\
\hline $1517 \mathrm{BC}$ & $14.7 \pm 2.2$ & $3251 \pm 17$ & 3466 \\
\hline $1527 \mathrm{BC}$ & $13.2 \pm 2.0$ & $3273 \pm 16$ & 3476 \\
\hline $1537 \mathrm{BC}$ & $10.0 \pm 2.1$ & $3308 \pm 17$ & 3486 \\
\hline $1547 \mathrm{BC}$ & $11.7 \pm 2.2$ & $3304 \pm 17$ & 3496 \\
\hline $1557 \mathrm{BC}$ & $12.9 \pm 2.1$ & $3304 \pm 17$ & 3506 \\
\hline $1567 \mathrm{BC}$ & $12.9 \pm 3.6$ & $3314 \pm 29$ & 3516 \\
\hline $1577 \mathrm{BC}$ & $17.3 \pm 3.6$ & $3289 \pm 29$ & 3526 \\
\hline $1587 \mathrm{BC}$ & $16.9 \pm 2.2$ & $3302 \pm 18$ & 3536 \\
\hline 1597 вС & $21.4 \pm 1.7$ & $3276 \pm 14$ & 3546 \\
\hline $1607 \mathrm{BC}$ & $16.4 \pm 1.6$ & $3326 \pm 13$ & 3556 \\
\hline $1617 \mathrm{BC}$ & $17.5 \pm 1.7$ & $3327 \pm 14$ & 3566 \\
\hline $1627 \mathrm{BC}$ & $15.7 \pm 1.7$ & $3350 \pm 13$ & 3576 \\
\hline $1637 \mathrm{BC}$ & $16.3 \pm 2.2$ & $3355 \pm 18$ & 3586 \\
\hline $1647 \mathrm{BC}$ & $13.5 \pm 1.3$ & $3387 \pm 10$ & 3596 \\
\hline $1657 \mathrm{BC}$ & $20.1 \pm 1.7$ & $3344 \pm 13$ & 3606 \\
\hline $1667 \mathrm{BC}$ & $13.5 \pm 2.3$ & $3406 \pm 18$ & 3616 \\
\hline 1677 вС & $25.8 \pm 2.2$ & $3320 \pm 17$ & 3626 \\
\hline $1687 \mathrm{BC}$ & $21.9 \pm 2.2$ & $3360 \pm 17$ & 3636 \\
\hline $1697 \mathrm{BC}$ & $13.7 \pm 1.5$ & $3434 \pm 12$ & 3646 \\
\hline $1707 \mathrm{BC}$ & $15.9 \pm 2.2$ & $3427 \pm 18$ & 3656 \\
\hline $1717 \mathrm{BC}$ & $20.8 \pm 2.2$ & $3398 \pm 17$ & 3666 \\
\hline $1727 \mathrm{BC}$ & $20.8 \pm 2.2$ & $3407 \pm 17$ & 3676 \\
\hline $1737 \mathrm{BC}$ & $19.1 \pm 2.2$ & $3430 \pm 17$ & 3686 \\
\hline $1747 \mathrm{BC}$ & $15.4 \pm 2.2$ & $3469 \pm 18$ & 3696 \\
\hline $1757 \mathrm{BC}$ & $14.6 \pm 1.6$ & $3485 \pm 13$ & 3706 \\
\hline $1767 \mathrm{BC}$ & $19.1 \pm 1.7$ & $3459 \pm 14$ & 3716 \\
\hline $1777 \mathrm{BC}$ & $15.4 \pm 1.7$ & $3498 \pm 13$ & 3726 \\
\hline $1787 \mathrm{BC}$ & $14.7 \pm 1.5$ & $3515 \pm 12$ & 3736 \\
\hline $1797 \mathrm{BC}$ & $17.4 \pm 1.5$ & $3501 \pm 12$ & 3746 \\
\hline $1807 \mathrm{BC}$ & $21.9 \pm 1.5$ & $3478 \pm 12$ & 3756 \\
\hline $1817 \mathrm{BC}$ & $19.0 \pm 1.5$ & $3509 \pm 12$ & 3766 \\
\hline $1827 \mathrm{BC}$ & $22.5 \pm 2.2$ & $3491 \pm 18$ & 3776 \\
\hline $1837 \mathrm{BC}$ & $15.9 \pm 2.1$ & $3553 \pm 17$ & 3786 \\
\hline $1847 \mathrm{BC}$ & $25.5 \pm 2.2$ & $3487 \pm 18$ & 3796 \\
\hline $1857 \mathrm{BC}$ & $27.5 \pm 2.2$ & $3481 \pm 18$ & 3806 \\
\hline $1867 \mathrm{BC}$ & $26.2 \pm 2.2$ & $3501 \pm 18$ & 3816 \\
\hline $1877 \mathrm{BC}$ & $27.1 \pm 1.6$ & $3503 \pm 12$ & 3826 \\
\hline 1887 ВС & $21.4 \pm 2.1$ & $3558 \pm 17$ & 3836 \\
\hline 1897 ВС & $19.9 \pm 2.2$ & $3580 \pm 18$ & 3846 \\
\hline
\end{tabular}


TABLE 1. Decadal Measurements (Continued)

\begin{tabular}{|c|c|c|c|}
\hline $\mathrm{cal} \mathrm{AD} / \mathrm{BC}$ & $\Delta^{14} \mathrm{C}(\% \circ)$ & ${ }^{14} \mathrm{C}$ BP & cal BP \\
\hline $1907 \mathrm{BC}$ & $24.2 \pm 2.2$ & $3555 \pm 17$ & 3856 \\
\hline $1917 \mathrm{BC}$ & $25.2 \pm 2.2$ & $3557 \pm 17$ & 3866 \\
\hline $1927 \mathrm{BC}$ & $21.1 \pm 1.7$ & $3599 \pm 14$ & 3876 \\
\hline 1937 ВС & $22.9 \pm 2.0$ & $3595 \pm 16$ & 3886 \\
\hline 1947 ВС & $22.4 \pm 2.3$ & $3609 \pm 18$ & 3896 \\
\hline $1957 \mathrm{BC}$ & $23.2 \pm 2.2$ & $3612 \pm 18$ & 3906 \\
\hline $1967 \mathrm{BC}$ & $23.5 \pm 2.2$ & $3619 \pm 18$ & 3916 \\
\hline $1977 \mathrm{BC}$ & $22.3 \pm 2.2$ & $3638 \pm 17$ & 3926 \\
\hline $1987 \mathrm{BC}$ & $19.5 \pm 2.2$ & $3670 \pm 17$ & 3936 \\
\hline 1997 ВС & $28.8 \pm 2.2$ & $3607 \pm 17$ & 3946 \\
\hline $2007 \mathrm{BC}$ & $32.1 \pm 2.3$ & $3591 \pm 18$ & 3956 \\
\hline $2017 \mathrm{BC}$ & $26.4 \pm 2.2$ & $3645 \pm 17$ & 3966 \\
\hline $2027 \mathrm{BC}$ & $28.8 \pm 2.2$ & $3636 \pm 17$ & 3976 \\
\hline $2037 \mathrm{BC}$ & $24.3 \pm 1.4$ & $3681 \pm 11$ & 3986 \\
\hline $2047 \mathrm{BC}$ & $22.6 \pm 1.6$ & $3704 \pm 12$ & 3996 \\
\hline $2057 \mathrm{BC}$ & $22.1 \pm 1.6$ & $3717 \pm 13$ & 4006 \\
\hline $2067 \mathrm{BC}$ & $28.4 \pm 2.2$ & $3678 \pm 17$ & 4016 \\
\hline $2077 \mathrm{BC}$ & $21.5 \pm 1.6$ & $3742 \pm 12$ & 4026 \\
\hline $2087 \mathrm{BC}$ & $32.4 \pm 2.2$ & $3666 \pm 17$ & 4036 \\
\hline $2097 \mathrm{BC}$ & $30.2 \pm 2.2$ & $3693 \pm 17$ & 4046 \\
\hline $2107 \mathrm{BC}$ & $35.2 \pm 1.6$ & $3664 \pm 12$ & 4056 \\
\hline $2117 \mathrm{BC}$ & $32.2 \pm 2.2$ & $3697 \pm 17$ & 4066 \\
\hline $2127 \mathrm{BC}$ & $35.1 \pm 2.2$ & $3684 \pm 17$ & 4076 \\
\hline $2137 \mathrm{BC}$ & $32.2 \pm 2.2$ & $3716 \pm 17$ & 4086 \\
\hline $2147 \mathrm{BC}$ & $27.5 \pm 1.4$ & $3762 \pm 11$ & 4096 \\
\hline $2157 \mathrm{BC}$ & $29.6 \pm 1.4$ & $3756 \pm 11$ & 4106 \\
\hline $2167 \mathrm{BC}$ & $32.6 \pm 2.3$ & $3743 \pm 18$ & 4116 \\
\hline $2177 \mathrm{BC}$ & $34.7 \pm 1.5$ & $3736 \pm 12$ & 4126 \\
\hline $2187 \mathrm{BC}$ & $36.6 \pm 1.3$ & $3730 \pm 10$ & 4136 \\
\hline 2197 BC & $36.0 \pm 1.3$ & $3745 \pm 10$ & 4146 \\
\hline $2207 \mathrm{BC}$ & $30.2 \pm 1.9$ & $3800 \pm 15$ & 4156 \\
\hline $2217 \mathrm{BC}$ & $30.2 \pm 1.6$ & $3811 \pm 12$ & 4166 \\
\hline $2227 \mathrm{BC}$ & \pm 1.3 & $3787 \pm 11$ & 4176 \\
\hline $2237 \mathrm{BC}$ & $28.9 \pm 2.5$ & $3839 \pm 19$ & 4186 \\
\hline $2247 \mathrm{BC}$ & $31.3 \pm 1.7$ & $3830 \pm 13$ & 4196 \\
\hline $2257 \mathrm{BC}$ & $37.2 \pm 1.6$ & $3793 \pm 12$ & 4206 \\
\hline $2267 \mathrm{BC}$ & $40.0 \pm 2.2$ & $2 \pm 17$ & 4216 \\
\hline $2277 \mathrm{BC}$ & \pm 2.7 & $3820 \pm 21$ & 4226 \\
\hline $2287 \mathrm{BC}$ & $37.7 \pm 1.9$ & $3820 \pm 15$ & 4236 \\
\hline $2297 \mathrm{BC}$ & $34.7 \pm 1.7$ & $3852 \pm 13$ & 4246 \\
\hline 2307 BC & $38.4 \pm 2.5$ & $3833 \pm 19$ & 425 \\
\hline $2317 \mathrm{BC}$ & \pm 2.3 & $3865 \pm 18$ & 4266 \\
\hline 2327 BC & $39.3 \pm 1.6$ & $3846 \pm 12$ & 4276 \\
\hline $2337 \mathrm{BC}$ & $38.9 \pm 2.3$ & $3859 \pm 17$ & 4286 \\
\hline $2347 \mathrm{BC}$ & $38.0 \pm 1.6$ & $3876 \pm 13$ & 4296 \\
\hline $2357 \mathrm{BC}$ & $36.5 \pm 2.3$ & $7 \pm 18$ & 4306 \\
\hline $67 \mathrm{BC}$ & $5 \pm 1.6$ & $3899 \pm 13$ & 4316 \\
\hline 2377 BC & $39.1 \pm 2.3$ & $3896 \pm 18$ & 4326 \\
\hline $2387 \mathrm{BC}$ & $44.8 \pm 1.7$ & $3864 \pm 13$ & 4336 \\
\hline 2397 BC & $50.1 \pm 2.5$ & $3832 \pm 20$ & 4346 \\
\hline 2407 BC & \pm 1.7 & $3900 \pm 13$ & 4356 \\
\hline 2417 BC & $41.8 \pm 1.8$ & $3914 \pm 14$ & 4366 \\
\hline 2427 BC & $47.3 \pm 2.2$ & $3882 \pm 17$ & 4376 \\
\hline 2437 BC & $46.3 \pm 1.2$ & $3900 \pm 9$ & 4386 \\
\hline 2447 BC & $48.4 \pm 2.4$ & $3893 \pm 18$ & 4396 \\
\hline 2467 BC & 2.3 & $3916 \pm 18$ & 4416 \\
\hline 2477 BC & $37.2 \pm 1.6$ & $4009 \pm 13$ & 4426 \\
\hline 2487 BC & $41.2 \pm 1.7$ & $3987 \pm 13$ & 4436 \\
\hline 2497 BC & $35.8 \pm 2.4$ & $4039 \pm 19$ & 4446 \\
\hline 2507 BC & $33.3 \pm 1.7$ & $4067 \pm 13$ & 4456 \\
\hline
\end{tabular}

TABLE 1. Decadal Measurements (Continued)

\begin{tabular}{|c|c|c|c|}
\hline cal AD/BC & $\Delta^{14} \mathrm{C}(\% o)$ & ${ }^{14} \mathrm{C}$ BP & cal BP \\
\hline 2517 BC & $40.9 \pm 1.7$ & $4018 \pm 13$ & 4466 \\
\hline $2527 \mathrm{BC}$ & $44.6 \pm 1.2$ & $4000 \pm 9$ & 4476 \\
\hline $2537 \mathrm{BC}$ & $48.1 \pm 1.6$ & $3983 \pm 12$ & 4486 \\
\hline $2547 \mathrm{BC}$ & $48.6 \pm 1.6$ & $3988 \pm 12$ & 4496 \\
\hline $2557 \mathrm{BC}$ & $47.9 \pm 1.5$ & $4003 \pm 12$ & 4506 \\
\hline $2567 \mathrm{BC}$ & $46.4 \pm 1.4$ & $4024 \pm 11$ & 4516 \\
\hline $2577 \mathrm{BC}$ & $43.9 \pm 1.7$ & $4053 \pm 13$ & 4526 \\
\hline $2587 \mathrm{BC}$ & $40.8 \pm 1.4$ & $4088 \pm 11$ & 4536 \\
\hline $2597 \mathrm{BC}$ & $47.1 \pm 1.6$ & $4049 \pm 12$ & 4546 \\
\hline $2607 \mathrm{BC}$ & $40.3 \pm 2.4$ & $4110 \pm 19$ & 4556 \\
\hline 2617 BC & $49.8 \pm 1.6$ & $4047 \pm 12$ & 4566 \\
\hline $2627 \mathrm{BC}$ & $45.5 \pm 1.7$ & $4089 \pm 13$ & 4576 \\
\hline $2637 \mathrm{BC}$ & $44.3 \pm 1.4$ & $4108 \pm 11$ & 4586 \\
\hline $2647 \mathrm{BC}$ & $39.1 \pm 2.3$ & $4159 \pm 18$ & 4596 \\
\hline $2655 \mathrm{BC}$ & $50.6 \pm 2.4$ & $4078 \pm 18$ & 4604 \\
\hline $2665 \mathrm{BC}$ & $49.9 \pm 1.6$ & $4093 \pm 12$ & 4614 \\
\hline $2675 \mathrm{BC}$ & $47.8 \pm 1.6$ & $4119 \pm 12$ & 4624 \\
\hline $2685 \mathrm{BC}$ & $48.7 \pm 1.6$ & $4122 \pm 12$ & 4634 \\
\hline $2695 \mathrm{BC}$ & $48.2 \pm 2.4$ & $4136 \pm 18$ & 4644 \\
\hline $2705 \mathrm{BC}$ & $47.5 \pm 1.3$ & $4150 \pm 10$ & 4654 \\
\hline $2715 \mathrm{BC}$ & $45.4 \pm 2.4$ & $4177 \pm 19$ & 4664 \\
\hline $2725 \mathrm{BC}$ & $51.9 \pm 2.4$ & $4137 \pm 19$ & 4674 \\
\hline $2735 \mathrm{BC}$ & $49.9 \pm 2.0$ & $4161 \pm 15$ & 4684 \\
\hline $2745 \mathrm{BC}$ & $54.1 \pm 2.4$ & $4139 \pm 18$ & 4694 \\
\hline $2755 \mathrm{BC}$ & $54.5 \pm 2.4$ & $4145 \pm 18$ & 4704 \\
\hline 2765 BC & $47.4 \pm 2.6$ & $4210 \pm 20$ & 4714 \\
\hline $2775 \mathrm{BC}$ & $54.4 \pm 2.1$ & $4166 \pm 16$ & 4724 \\
\hline 2785 BC & $57.4 \pm 1.7$ & $4153 \pm 13$ & 4734 \\
\hline 2795 BC & $55.6 \pm 1.7$ & $4176 \pm 13$ & 4744 \\
\hline $2805 \mathrm{BC}$ & $53.7 \pm 2.3$ & $4201 \pm 17$ & 4754 \\
\hline $2815 \mathrm{BC}$ & $66.5 \pm 1.7$ & $4114 \pm 13$ & 4764 \\
\hline $2825 \mathrm{BC}$ & $72.3 \pm 2.6$ & $4079 \pm 19$ & 4774 \\
\hline 2835 BC & $67.6 \pm 2.3$ & \pm 17 & 4784 \\
\hline 2845 BC & $65.1 \pm 2.2$ & $4153 \pm 17$ & 4794 \\
\hline $2855 \mathrm{BC}$ & $66.7 \pm 2.3$ & $4151 \pm 18$ & 4804 \\
\hline $2865 \mathrm{BC}$ & $66.3 \pm 2.2$ & $4163 \pm 16$ & 4814 \\
\hline 2875 BC & $65.1 \pm 1.6$ & $4182 \pm 12$ & 4824 \\
\hline 2885 BC & \pm 2.3 & $4220 \pm 17$ & 4834 \\
\hline 2895 BC & $55.1 \pm 2.3$ & $4277 \pm 18$ & 4844 \\
\hline 2905 BC & $54.5 \pm 2.3$ & $4292 \pm 18$ & 4854 \\
\hline $2915 \mathrm{BC}$ & $53.4 \pm 2.2$ & $4310 \pm 17$ & 4864 \\
\hline $2925 \mathrm{BC}$ & $47.9 \pm 2.3$ & \pm 18 & 4874 \\
\hline $35 \mathrm{BC}$ & $46.9 \pm 2.6$ & $4379 \pm 20$ & 4884 \\
\hline 2945 BC & $45.3 \pm 2.2$ & $4401 \pm 17$ & 4894 \\
\hline $2955 \mathrm{BC}$ & $52.7 \pm 1.6$ & $4355 \pm 12$ & 4904 \\
\hline 2965 BC & $52.2 \pm 2.2$ & $4367 \pm 17$ & 4914 \\
\hline $975 \mathrm{BC}$ & $50.2 \pm 2.3$ & $4392 \pm 18$ & 4924 \\
\hline 2985 BC & $56.8 \pm 1.6$ & $4352 \pm 12$ & 4934 \\
\hline 2995 BC & $59.7 \pm 2.4$ & $4339 \pm 18$ & 4944 \\
\hline $3005 \mathrm{BC}$ & $58.4 \pm 2.4$ & $4359 \pm 18$ & 4954 \\
\hline $3015 \mathrm{BC}$ & $57.5 \pm 2.3$ & $4376 \pm 18$ & 4964 \\
\hline $3025 \mathrm{BC}$ & $59.2 \pm 2.3$ & $4372 \pm 18$ & 4974 \\
\hline 3035 BC & $59.2 \pm 2.4$ & $4382 \pm 18$ & 4984 \\
\hline 3045 BC & $55.7 \pm 1.5$ & $4418 \pm 12$ & 4994 \\
\hline 3055 BC & $54.8 \pm 1.6$ & $4435 \pm 12$ & 5004 \\
\hline & $56.5 \pm 2.4$ & $4432 \pm 18$ & 5014 \\
\hline $3075 \mathrm{BC}$ & $63.2 \pm 1.2$ & $4390 \pm 9$ & 5024 \\
\hline 3085 BC & $58.8 \pm 2.4$ & $4434 \pm 18$ & 5034 \\
\hline & & $4428 \pm 18$ & 5044 \\
\hline & $52.3 \pm 2.4$ & $4503 \pm 18$ & 5054 \\
\hline
\end{tabular}


TABLE 1. Decadal Measurements (Continued)

\begin{tabular}{|c|c|c|c|}
\hline cal $\mathrm{AD} / \mathrm{BC}$ & $\Delta^{14} \mathrm{C}(\% \circ)$ & ${ }^{14} \mathrm{C}$ BP & cal BP \\
\hline $3115 \mathrm{BC}$ & $56.5 \pm 1.7$ & $4481 \pm 13$ & 5064 \\
\hline $3125 \mathrm{BC}$ & $53.7 \pm 2.3$ & $4512 \pm 18$ & 5074 \\
\hline $3135 \mathrm{BC}$ & $55.1 \pm 2.3$ & $4510 \pm 17$ & 5084 \\
\hline 3145 вC & $51.9 \pm 2.3$ & $4544 \pm 18$ & 5094 \\
\hline $3155 \mathrm{BC}$ & $58.9 \pm 2.4$ & $4501 \pm 18$ & 5104 \\
\hline $3165 \mathrm{BC}$ & $64.7 \pm 1.7$ & $4467 \pm 13$ & 5114 \\
\hline $3175 \mathrm{BC}$ & $58.9 \pm 2.4$ & $4520 \pm 18$ & 5124 \\
\hline $3185 \mathrm{BC}$ & $63.0 \pm 1.7$ & $4500 \pm 13$ & 5134 \\
\hline $3195 \mathrm{BC}$ & $61.5 \pm 1.7$ & $4520 \pm 12$ & 5144 \\
\hline $3205 \mathrm{BC}$ & $61.7 \pm 2.4$ & $4528 \pm 18$ & 5154 \\
\hline $3215 \mathrm{BC}$ & $67.0 \pm 1.5$ & $4497 \pm 11$ & 5164 \\
\hline $3225 \mathrm{BC}$ & $68.7 \pm 2.4$ & $4495 \pm 18$ & 5174 \\
\hline $3235 \mathrm{BC}$ & $74.5 \pm 2.4$ & $4461 \pm 18$ & 5184 \\
\hline $3245 \mathrm{BC}$ & $79.3 \pm 2.4$ & $4435 \pm 18$ & 5194 \\
\hline $3255 \mathrm{BC}$ & $77.9 \pm 2.4$ & $4455 \pm 18$ & 5204 \\
\hline $3265 \mathrm{BC}$ & $75.1 \pm 2.1$ & $4486 \pm 16$ & 5214 \\
\hline $3275 \mathrm{BC}$ & $77.2 \pm 2.6$ & $4480 \pm 19$ & 5224 \\
\hline $3285 \mathrm{BC}$ & $80.0 \pm 2.4$ & $4469 \pm 18$ & 5234 \\
\hline $3295 \mathrm{BC}$ & $81.4 \pm 2.4$ & $4468 \pm 18$ & 5244 \\
\hline 3305 BC & $81.0 \pm 2.5$ & $4480 \pm 18$ & 5254 \\
\hline $3315 \mathrm{BC}$ & $78.4 \pm 1.8$ & $4511 \pm 13$ & 5264 \\
\hline $3325 \mathrm{BC}$ & $84.2 \pm 1.7$ & $4476 \pm 13$ & 5274 \\
\hline $3335 \mathrm{BC}$ & $78.9 \pm 1.6$ & $4525 \pm 12$ & 5284 \\
\hline $3345 \mathrm{BC}$ & $78.8 \pm 1.6$ & $4535 \pm 12$ & 5294 \\
\hline $3355 \mathrm{BC}$ & $74.9 \pm 2.5$ & $4575 \pm 19$ & 5304 \\
\hline $3365 \mathrm{BC}$ & $72.0 \pm 2.4$ & $4606 \pm 18$ & 5314 \\
\hline 3375 вС & $68.2 \pm 2.4$ & $4644 \pm 18$ & 5324 \\
\hline $3385 \mathrm{BC}$ & $63.7 \pm 2.4$ & $4688 \pm 18$ & 5334 \\
\hline $3395 \mathrm{BC}$ & $60.8 \pm 1.8$ & $4718 \pm 14$ & 5344 \\
\hline $3405 \mathrm{BC}$ & $66.8 \pm 2.4$ & $4684 \pm 18$ & 5354 \\
\hline $3415 \mathrm{BC}$ & $65.6 \pm 2.7$ & $4703 \pm 20$ & 5364 \\
\hline $3425 \mathrm{BC}$ & $70.9 \pm 2.0$ & $4673 \pm 15$ & 5374 \\
\hline $3435 \mathrm{BC}$ & $73.4 \pm 1.7$ & $4664 \pm 13$ & 5384 \\
\hline $3445 \mathrm{BC}$ & $73.8 \pm 2.9$ & $4670 \pm 22$ & 5394 \\
\hline $3455 \mathrm{BC}$ & $74.0 \pm 2.7$ & $4679 \pm 20$ & 5404 \\
\hline $3465 \mathrm{BC}$ & $80.0 \pm 1.7$ & $4643 \pm 13$ & 5414 \\
\hline $3475 \mathrm{BC}$ & $86.6 \pm 1.7$ & $4604 \pm 13$ & 5424 \\
\hline $3485 \mathrm{BC}$ & $87.1 \pm 1.7$ & $4611 \pm 13$ & 5434 \\
\hline $3495 \mathrm{BC}$ & $86.1 \pm 1.8$ & $4628 \pm 13$ & 5444 \\
\hline $3505 \mathrm{BC}$ & $79.9 \pm 1.8$ & $4684 \pm 13$ & 5454 \\
\hline $3515 \mathrm{BC}$ & $76.3 \pm 1.7$ & $4720 \pm 13$ & 5464 \\
\hline $3525 \mathrm{BC}$ & $77.2 \pm 1.8$ & $4723 \pm 13$ & 5474 \\
\hline $3535 \mathrm{BC}$ & $78.4 \pm 1.7$ & $4725 \pm 13$ & 5484 \\
\hline $3545 \mathrm{BC}$ & $73.1 \pm 1.7$ & $4773 \pm 13$ & 5494 \\
\hline $3555 \mathrm{BC}$ & $74.9 \pm 1.8$ & $4769 \pm 13$ & 5504 \\
\hline $3565 \mathrm{BC}$ & $79.7 \pm 1.8$ & $4743 \pm 13$ & 5514 \\
\hline $3575 \mathrm{BC}$ & $79.5 \pm 1.8$ & $4755 \pm 13$ & 5524 \\
\hline $3585 \mathrm{BC}$ & $82.7 \pm 1.8$ & $4741 \pm 13$ & 5534 \\
\hline $3595 \mathrm{BC}$ & $85.0 \pm 1.4$ & $4734 \pm 10$ & 5544 \\
\hline $3605 \mathrm{BC}$ & $88.1 \pm 1.6$ & $4719 \pm 12$ & 5554 \\
\hline $3615 \mathrm{BC}$ & $91.3 \pm 1.4$ & $4706 \pm 10$ & 5564 \\
\hline $3625 \mathrm{BC}$ & $89.4 \pm 2.5$ & $4730 \pm 19$ & 5574 \\
\hline $3635 \mathrm{BC}$ & $82.5 \pm 1.7$ & $4790 \pm 13$ & 5584 \\
\hline $3645 \mathrm{BC}$ & $77.4 \pm 1.8$ & $4837 \pm 13$ & 5594 \\
\hline $3655 \mathrm{BC}$ & $73.4 \pm 1.7$ & $4878 \pm 13$ & 5604 \\
\hline $3665 \mathrm{BC}$ & $75.2 \pm 1.7$ & $4874 \pm 13$ & 5614 \\
\hline $3675 \mathrm{BC}$ & $69.7 \pm 1.4$ & $4925 \pm 11$ & 5624 \\
\hline $3685 \mathrm{BC}$ & $75.0 \pm 1.7$ & $4895 \pm 13$ & 5634 \\
\hline $3695 \mathrm{BC}$ & $77.3 \pm 1.7$ & $4888 \pm 13$ & 5644 \\
\hline $3705 \mathrm{BC}$ & $74.8 \pm 1.7$ & $4916 \pm 13$ & 5654 \\
\hline
\end{tabular}

TABLE 1. Decadal Measurements (Continued)

\begin{tabular}{|c|c|c|c|}
\hline cal $\mathrm{AD} / \mathrm{BC}$ & $\Delta^{14} \mathrm{C}(\% \circ)$ & ${ }^{14} \mathrm{C}$ BP & cal BP \\
\hline $3715 \mathrm{BC}$ & $71.4 \pm 1.7$ & $4951 \pm 13$ & 5664 \\
\hline 3725 BC & $71.4 \pm 2.7$ & $4961 \pm 20$ & 5674 \\
\hline $3735 \mathrm{BC}$ & $75.9 \pm 1.8$ & $4937 \pm 13$ & 5684 \\
\hline $3745 \mathrm{BC}$ & $76.0 \pm 1.5$ & $4946 \pm 12$ & 5694 \\
\hline $3755 \mathrm{BC}$ & $76.9 \pm 1.7$ & $4949 \pm 13$ & 5704 \\
\hline $3765 \mathrm{BC}$ & $72.8 \pm 1.7$ & $4989 \pm 13$ & 5714 \\
\hline $3775 \mathrm{BC}$ & $73.2 \pm 1.8$ & $4995 \pm 13$ & 5724 \\
\hline $3785 \mathrm{BC}$ & $74.9 \pm 1.4$ & $4992 \pm 11$ & 5734 \\
\hline $3795 \mathrm{BC}$ & $75.8 \pm 1.8$ & $4996 \pm 14$ & 5744 \\
\hline $3805 \mathrm{BC}$ & $65.4 \pm 1.7$ & $5083 \pm 13$ & 5754 \\
\hline $3815 \mathrm{BC}$ & $65.5 \pm 2.3$ & $5092 \pm 17$ & 5764 \\
\hline $3825 \mathrm{BC}$ & $71.4 \pm 2.3$ & $5058 \pm 17$ & 5774 \\
\hline $3835 \mathrm{BC}$ & $73.1 \pm 2.4$ & $5055 \pm 18$ & 5784 \\
\hline 3845 BC & $71.5 \pm 2.4$ & $5077 \pm 18$ & 5794 \\
\hline $3855 \mathrm{BC}$ & $71.9 \pm 1.4$ & $5084 \pm 10$ & 5804 \\
\hline $3865 \mathrm{BC}$ & $78.1 \pm 1.2$ & $5046 \pm 10$ & 5814 \\
\hline 3875 BC & $73.9 \pm 1.3$ & $5088 \pm 10$ & 5824 \\
\hline $3885 \mathrm{BC}$ & $87.1 \pm 1.6$ & $4999 \pm 12$ & 5834 \\
\hline $3895 \mathrm{BC}$ & $82.5 \pm 2.1$ & $5044 \pm 15$ & 5844 \\
\hline $3905 \mathrm{BC}$ & $82.6 \pm 1.7$ & $5052 \pm 12$ & 5854 \\
\hline $3915 \mathrm{BC}$ & $81.9 \pm 1.7$ & $5067 \pm 12$ & 5864 \\
\hline $3925 \mathrm{BC}$ & $84.0 \pm 2.1$ & $5062 \pm 16$ & 5874 \\
\hline $3935 \mathrm{BC}$ & $85.1 \pm 2.0$ & $5063 \pm 15$ & 5884 \\
\hline 3945 BC & $81.1 \pm 1.7$ & $5102 \pm 13$ & 5894 \\
\hline $3955 \mathrm{BC}$ & $82.0 \pm 1.9$ & $5105 \pm 15$ & 5904 \\
\hline $3965 \mathrm{BC}$ & $76.1 \pm 1.2$ & $5159 \pm 9$ & 5914 \\
\hline $3975 \mathrm{BC}$ & $74.5 \pm 1.2$ & $5181 \pm$ & 5924 \\
\hline $3985 \mathrm{BC}$ & $70.3 \pm 1.6$ & $5221 \pm 12$ & 5934 \\
\hline 3995 ВС & \pm 1.7 & $5237 \pm 13$ & 5944 \\
\hline $4005 \mathrm{BC}$ & $69.0 \pm 1.8$ & $5251 \pm 13$ & 5954 \\
\hline $4015 \mathrm{BC}$ & $72.1 \pm 2.4$ & $5237 \pm 18$ & 5964 \\
\hline $4025 \mathrm{BC}$ & $77.2 \pm 1.4$ & $5209 \pm 11$ & 5974 \\
\hline $4035 \mathrm{BC}$ & $78.5 \pm 1.2$ & $5209 \pm 9$ & 5984 \\
\hline $4045 \mathrm{BC}$ & $73.7 \pm 1.8$ & $5255 \pm 13$ & 5994 \\
\hline $4085 \mathrm{BC}$ & $73.0 \pm 2.1$ & $5298 \pm 15$ & 6034 \\
\hline $4095 \mathrm{BC}$ & $74.6 \pm 1.7$ & $5296 \pm 13$ & 6044 \\
\hline 4105 BC & $78.4 \pm 2.5$ & $5278 \pm 18$ & 6054 \\
\hline $4115 \mathrm{BC}$ & \pm 1.7 & $5354 \pm 13$ & 6064 \\
\hline 4125 BC & $80.2 \pm 2.5$ & $5284 \pm 19$ & 6074 \\
\hline $4135 \mathrm{BC}$ & $82.0 \pm 1.8$ & $5280 \pm 13$ & 6084 \\
\hline $4155 \mathrm{BC}$ & $85.6 \pm 2.5$ & $5273 \pm 19$ & 6104 \\
\hline $4165 \mathrm{BC}$ & \pm 2.5 & $3 \pm 18$ & 6114 \\
\hline $4175 \mathrm{BC}$ & $75.3 \pm 2.6$ & $5369 \pm 19$ & 6124 \\
\hline 4185 BC & $81.4 \pm 2.5$ & $5333 \pm 19$ & 6134 \\
\hline $4195 \mathrm{BC}$ & $84.8 \pm 1.8$ & $5318 \pm 13$ & 6144 \\
\hline 4205 BC & $92.6 \pm 2.4$ & $5270 \pm 18$ & 6154 \\
\hline $4215 \mathrm{BC}$ & \pm 2.6 & $5269 \pm 19$ & 6164 \\
\hline 4225 вC & $81.5 \pm 1.8$ & $5372 \pm 13$ & 6174 \\
\hline 4235 вС & $81.5 \pm 1.8$ & $5381 \pm 13$ & 6184 \\
\hline 4245 BC & $86.3 \pm 1.8$ & $5355 \pm 13$ & 6194 \\
\hline 4255 вС & & & 6204 \\
\hline 4265 BC & $74.8 \pm 1.9$ & $5460 \pm 14$ & 6214 \\
\hline $4275 \mathrm{BC}$ & $79.7 \pm 3.0$ & $5433 \pm 22$ & 6224 \\
\hline $4285 \mathrm{BC}$ & $81.2 \pm 1.8$ & $5433 \pm 13$ & 6234 \\
\hline 4295 BC & $85.5 \pm 2.6$ & $5409 \pm 20$ & 6244 \\
\hline 4305 BC & $91.6 \pm 2.5$ & $5374 \pm 19$ & 6254 \\
\hline $4315 \mathrm{BC}$ & $92.1 \pm 2.6$ & $5380 \pm 19$ & 6264 \\
\hline $4325 \mathrm{BC}$ & $86.7 \pm 2.6$ & $5430 \pm 19$ & 6274 \\
\hline & $80.7 \pm 1.8$ & $5484 \pm 13$ & 6284 \\
\hline & & $5513 \pm 13$ & 6294 \\
\hline
\end{tabular}


TABLE 1. Decadal Measurements (Continued)

\begin{tabular}{|c|c|c|c|}
\hline cal $\mathrm{AD} / \mathrm{BC}$ & $\Delta^{14} \mathrm{C}(\% o)$ & ${ }^{14} \mathrm{C} B P$ & cal BP \\
\hline $4355 \mathrm{BC}$ & $74.0 \pm 2.6$ & $5553 \pm 19$ & 6304 \\
\hline $4365 \mathrm{BC}$ & $73.8 \pm 2.5$ & $5564 \pm 19$ & 6314 \\
\hline $4375 \mathrm{BC}$ & $73.4 \pm 1.8$ & $5575 \pm 13$ & 6324 \\
\hline $4385 \mathrm{BC}$ & $78.7 \pm 2.6$ & $5547 \pm 20$ & 6334 \\
\hline $4395 \mathrm{BC}$ & $75.4 \pm 2.6$ & $5582 \pm 20$ & 6344 \\
\hline $4405 \mathrm{BC}$ & $77.7 \pm 2.6$ & $5574 \pm 19$ & 6354 \\
\hline $4415 \mathrm{BC}$ & $73.4 \pm 2.6$ & $5616 \pm 19$ & 6364 \\
\hline $4425 \mathrm{BC}$ & $84.7 \pm 2.7$ & $5542 \pm 20$ & 6374 \\
\hline 4435 вС & $87.9 \pm 2.7$ & $5527 \pm 20$ & 6384 \\
\hline 4445 BC & $83.6 \pm 2.6$ & $5569 \pm 19$ & 6394 \\
\hline $4455 \mathrm{BC}$ & $79.7 \pm 2.7$ & $5608 \pm 20$ & 6404 \\
\hline $4465 \mathrm{BC}$ & $71.5 \pm 1.8$ & $5679 \pm 13$ & 6414 \\
\hline 4475 BC & $73.4 \pm 2.6$ & $5675 \pm 19$ & 6424 \\
\hline $4485 \mathrm{BC}$ & $76.3 \pm 2.7$ & $5663 \pm 20$ & 6434 \\
\hline $4495 \mathrm{BC}$ & $75.5 \pm 2.6$ & $5678 \pm 19$ & 6444 \\
\hline $4505 \mathrm{BC}$ & $76.5 \pm 2.5$ & $5681 \pm 19$ & 6454 \\
\hline $4515 \mathrm{BC}$ & $77.3 \pm 2.6$ & $5684 \pm 19$ & 6464 \\
\hline $4525 \mathrm{BC}$ & $78.7 \pm 2.8$ & $5684 \pm 21$ & 6474 \\
\hline $4535 \mathrm{BC}$ & $79.1 \pm 2.6$ & $5690 \pm 19$ & 6484 \\
\hline $4545 \mathrm{BC}$ & $72.6 \pm 2.7$ & $5748 \pm 21$ & 6494 \\
\hline $4555 \mathrm{BC}$ & $72.9 \pm 2.6$ & $5756 \pm 19$ & 6504 \\
\hline $4565 \mathrm{BC}$ & $72.7 \pm 1.9$ & $5767 \pm 14$ & 6514 \\
\hline $4575 \mathrm{BC}$ & $76.0 \pm 1.8$ & $5753 \pm 13$ & 6524 \\
\hline $4585 \mathrm{BC}$ & $77.2 \pm 1.8$ & $5753 \pm 13$ & 6534 \\
\hline $4595 \mathrm{BC}$ & $76.5 \pm 2.6$ & $5768 \pm 19$ & 6544 \\
\hline $4605 \mathrm{BC}$ & $78.0 \pm 2.5$ & $5767 \pm 19$ & 6554 \\
\hline $4615 \mathrm{BC}$ & $79.7 \pm 2.8$ & $5764 \pm 21$ & 6564 \\
\hline $4625 \mathrm{BC}$ & $74.8 \pm 1.6$ & $5810 \pm 12$ & 6574 \\
\hline $4635 \mathrm{BC}$ & $79.0 \pm 2.2$ & $5788 \pm 16$ & 6584 \\
\hline $4645 \mathrm{BC}$ & $82.0 \pm 1.3$ & $5775 \pm 10$ & 6594 \\
\hline $4655 \mathrm{BC}$ & $82.2 \pm 1.8$ & $5784 \pm 13$ & 6604 \\
\hline $4665 \mathrm{BC}$ & $87.5 \pm 2.6$ & $5754 \pm 19$ & 6614 \\
\hline $4675 \mathrm{BC}$ & $87.4 \pm 2.8$ & $5764 \pm 21$ & 6624 \\
\hline $4685 \mathrm{BC}$ & $86.3 \pm 1.5$ & $5782 \pm 11$ & 6634 \\
\hline $4695 \mathrm{BC}$ & $80.3 \pm 1.9$ & $5837 \pm 14$ & 6644 \\
\hline $4705 \mathrm{BC}$ & $83.7 \pm 1.8$ & $5822 \pm 13$ & 6654 \\
\hline $4715 \mathrm{BC}$ & $79.9 \pm 2.5$ & $5859 \pm 19$ & 6664 \\
\hline $4725 \mathrm{BC}$ & $78.5 \pm 2.6$ & $5879 \pm 19$ & 6674 \\
\hline $4735 \mathrm{BC}$ & $80.1 \pm 2.5$ & $5877 \pm 19$ & 6684 \\
\hline $4895 \mathrm{BC}$ & $86.6 \pm 1.9$ & $5985 \pm 14$ & 6844 \\
\hline $4905 \mathrm{BC}$ & $88.1 \pm 1.5$ & $5984 \pm 11$ & 6854 \\
\hline $4915 \mathrm{BC}$ & $85.1 \pm 2.1$ & $6015 \pm 15$ & 6864 \\
\hline $4925 \mathrm{BC}$ & $83.9 \pm 2.6$ & $6033 \pm 20$ & 6874 \\
\hline $4935 \mathrm{BC}$ & $90.0 \pm 1.9$ & $5999 \pm 14$ & 6884 \\
\hline 4945 вС & $84.5 \pm 1.4$ & $6048 \pm 10$ & 6894 \\
\hline $4955 \mathrm{BC}$ & $86.6 \pm 1.8$ & $6042 \pm 14$ & 6904 \\
\hline $4965 \mathrm{BC}$ & $84.9 \pm 1.9$ & $6066 \pm 14$ & 6914 \\
\hline $4975 \mathrm{BC}$ & $87.8 \pm 1.8$ & $6053 \pm 13$ & 6924 \\
\hline $4985 \mathrm{BC}$ & $88.5 \pm 2.0$ & $6058 \pm 14$ & 6934 \\
\hline $4995 \mathrm{BC}$ & $85.2 \pm 2.6$ & $6092 \pm 19$ & 6944 \\
\hline $5005 \mathrm{BC}$ & $83.4 \pm 2.6$ & $6115 \pm 19$ & 6954 \\
\hline $5015 \mathrm{BC}$ & $88.5 \pm 2.7$ & $6087 \pm 20$ & 6964 \\
\hline 5025 вС & $85.8 \pm 2.6$ & $6117 \pm 19$ & 6974 \\
\hline 5035 вC & $87.3 \pm 2.7$ & $6115 \pm 20$ & 6984 \\
\hline 5045 вС & $85.5 \pm 2.7$ & $6138 \pm 20$ & 6994 \\
\hline $5055 \mathrm{BC}$ & $89.4 \pm 2.7$ & $6119 \pm 20$ & 7004 \\
\hline $5065 \mathrm{BC}$ & $83.5 \pm 2.6$ & $6172 \pm 19$ & 7014 \\
\hline $5075 \mathrm{BC}$ & $82.5 \pm 2.7$ & $6189 \pm 20$ & 7024 \\
\hline 5085 вС & $80.4 \pm 2.6$ & $6215 \pm 19$ & 7034 \\
\hline $5095 \mathrm{BC}$ & $87.0 \pm 1.9$ & $6176 \pm 14$ & 7044 \\
\hline
\end{tabular}

TABLE 1. Decadal Measurements (Continued)

\begin{tabular}{rrcr}
\hline cal AD/BC & \multicolumn{1}{c}{$\Delta^{14} \mathrm{C}(\%)$} & \multicolumn{1}{c}{$14 \mathrm{CP}$} & cal BP \\
\hline $5105 \mathrm{BC}$ & $83.0 \pm 2.6$ & $6215 \pm 19$ & 7054 \\
$5115 \mathrm{BC}$ & $92.7 \pm 2.5$ & $6153 \pm 19$ & 7064 \\
$5125 \mathrm{BC}$ & $85.1 \pm 2.6$ & $6219 \pm 19$ & 7074 \\
$5135 \mathrm{BC}$ & $94.9 \pm 2.5$ & $6157 \pm 18$ & 7084 \\
$5145 \mathrm{BC}$ & $95.7 \pm 1.9$ & $6160 \pm 14$ & 7094 \\
$5165 \mathrm{BC}$ & $92.7 \pm 2.6$ & $6202 \pm 19$ & 7114 \\
$5175 \mathrm{BC}$ & $99.1 \pm 2.6$ & $6164 \pm 19$ & 7124 \\
$5185 \mathrm{BC}$ & $106.8 \pm 1.9$ & $6119 \pm 14$ & 7134 \\
$5195 \mathrm{BC}$ & $106.7 \pm 2.6$ & $6128 \pm 19$ & 7144 \\
$5205 \mathrm{BC}$ & $109.0 \pm 2.8$ & $6122 \pm 20$ & 7154 \\
$5266 \mathrm{BC}$ & $95.2 \pm 2.8$ & $6281 \pm 21$ & 7215 \\
$5276 \mathrm{BC}$ & $103.6 \pm 2.7$ & $6230 \pm 20$ & 7225 \\
$5286 \mathrm{BC}$ & $101.5 \pm 1.9$ & $6255 \pm 14$ & 7235 \\
$5296 \mathrm{BC}$ & $102.7 \pm 1.9$ & $6256 \pm 14$ & 7245 \\
$5306 \mathrm{BC}$ & $97.9 \pm 2.6$ & $6300 \pm 19$ & 7255 \\
$5316 \mathrm{BC}$ & $96.4 \pm 1.7$ & $6321 \pm 13$ & 7265 \\
$5326 \mathrm{BC}$ & $88.5 \pm 1.8$ & $6389 \pm 13$ & 7275 \\
$5336 \mathrm{BC}$ & $94.9 \pm 2.7$ & $6352 \pm 20$ & 7285 \\
$5346 \mathrm{BC}$ & $92.2 \pm 2.8$ & $6381 \pm 20$ & 7295 \\
$5356 \mathrm{BC}$ & $96.2 \pm 1.9$ & $6361 \pm 14$ & 7305 \\
$5366 \mathrm{BC}$ & $94.8 \pm 1.9$ & $6383 \pm 14$ & 7315 \\
$5376 \mathrm{BC}$ & $90.6 \pm 2.6$ & $6422 \pm 19$ & 7325 \\
$5386 \mathrm{BC}$ & $91.2 \pm 1.9$ & $6427 \pm 15$ & 7335 \\
$5396 \mathrm{BC}$ & $88.0 \pm 1.9$ & $6462 \pm 15$ & 7345 \\
$5406 \mathrm{BC}$ & $96.5 \pm 2.7$ & $6408 \pm 20$ & 7355 \\
$5416 \mathrm{BC}$ & $100.3 \pm 2.9$ & $6390 \pm 21$ & 7365 \\
$5426 \mathrm{BC}$ & $98.7 \pm 3.1$ & $6412 \pm 23$ & 7375 \\
$5436 \mathrm{BC}$ & $101.5 \pm 2.7$ & $6400 \pm 20$ & 7385 \\
$5446 \mathrm{BC}$ & $100.7 \pm 2.7$ & $6416 \pm 20$ & 7395 \\
$5456 \mathrm{BC}$ & $106.5 \pm 1.9$ & $6384 \pm 14$ & 7405 \\
$5466 \mathrm{BC}$ & $105.4 \pm 2.1$ & $6402 \pm 15$ & 7415 \\
$5476 \mathrm{BC}$ & $95.2 \pm 2.6$ & $6485 \pm 19$ & 7425 \\
$5486 \mathrm{BC}$ & $85.4 \pm 2.0$ & $6568 \pm 15$ & 7435 \\
$5496 \mathrm{BC}$ & $87.2 \pm 2.7$ & $6564 \pm 20$ & 7445 \\
$5506 \mathrm{BC}$ & $92.6 \pm 1.9$ & $6534 \pm 14$ & 7455 \\
$5736 \mathrm{BC}$ & $74.4 \pm 2.7$ & $6892 \pm 20$ & 7685 \\
$5756 \mathrm{BC}$ & $74.0 \pm 1.9$ & $6905 \pm 14$ & 7695 \\
$5516 \mathrm{BC}$ & $89.2 \pm 2.8$ & $6568 \pm 20$ & 7465 \\
$5526 \mathrm{BC}$ & $92.5 \pm 2.8$ & $6554 \pm 21$ & 7475 \\
$5536 \mathrm{BC}$ & $88.5 \pm 2.8$ & $6593 \pm 20$ & 7485 \\
$5706 \mathrm{BC}$ & $84017 \pm 21$ & 7705
\end{tabular}


TABLE 1. Decadal Measurements (Continued)

\begin{tabular}{|c|c|c|c|}
\hline cal AD/BC & $\Delta^{14} \mathrm{C}(\% o)$ & ${ }^{14} \mathrm{C}$ BP & cal BP \\
\hline $5766 \mathrm{BC}$ & $76.9 \pm 2.5$ & $6903 \pm 19$ & 7715 \\
\hline 5776 вС & $76.3 \pm 2.4$ & $6917 \pm 18$ & 7725 \\
\hline 5786 вС & $77.5 \pm 2.5$ & $6918 \pm 19$ & 7735 \\
\hline 5796 вС & $77.4 \pm 2.8$ & $6928 \pm 21$ & 7745 \\
\hline 5806 вС & $80.0 \pm 2.0$ & $6918 \pm 15$ & 7755 \\
\hline 5816 BC & $76.5 \pm 2.8$ & $6955 \pm 21$ & 7765 \\
\hline $5826 \mathrm{BC}$ & $81.7 \pm 1.9$ & $6926 \pm 14$ & 7775 \\
\hline 5836 вС & $85.5 \pm 2.0$ & $6906 \pm 15$ & 7785 \\
\hline $5846 \mathrm{BC}$ & $77.7 \pm 2.8$ & $6975 \pm 21$ & 7795 \\
\hline $5856 \mathrm{BC}$ & $78.3 \pm 2.0$ & $6980 \pm 15$ & 7805 \\
\hline $5866 \mathrm{BC}$ & $82.5 \pm 1.9$ & $6958 \pm 14$ & 7815 \\
\hline 5876 вС & $82.5 \pm 1.9$ & $6968 \pm 14$ & 7825 \\
\hline $5886 \mathrm{BC}$ & $83.6 \pm 1.9$ & $6969 \pm 15$ & 7835 \\
\hline 5896 вС & $76.1 \pm 3.0$ & $7035 \pm 22$ & 7845 \\
\hline $5906 \mathrm{BC}$ & $81.5 \pm 2.4$ & $7005 \pm 18$ & 7855 \\
\hline $5916 \mathrm{BC}$ & $81.8 \pm 2.7$ & $7012 \pm 20$ & 7865 \\
\hline $5926 \mathrm{BC}$ & $74.4 \pm 2.7$ & $7077 \pm 20$ & 7875 \\
\hline $5936 \mathrm{BC}$ & $73.4 \pm 2.9$ & $7094 \pm 22$ & 7885 \\
\hline 5946 ВС & $80.3 \pm 1.9$ & $7053 \pm 15$ & 7895 \\
\hline $5956 \mathrm{BC}$ & $90.5 \pm 2.1$ & $6987 \pm 16$ & 7905 \\
\hline 5966 вС & $92.5 \pm 1.9$ & $6982 \pm 15$ & 7915 \\
\hline $5976 \mathrm{BC}$ & $87.0 \pm 3.3$ & $7032 \pm 25$ & 7925 \\
\hline $5986 \mathrm{BC}$ & $80.8 \pm 2.7$ & $7088 \pm 20$ & 7935 \\
\hline 5996 вС & $80.8 \pm 2.6$ & $7097 \pm 19$ & 7945 \\
\hline $6006 \mathrm{BC}$ & $79.3 \pm 2.8$ & $7118 \pm 21$ & 7955 \\
\hline 6016 BC & $75.7 \pm 3.2$ & $7155 \pm 24$ & 7965 \\
\hline $6023 \mathrm{BC}$ & $70.8 \pm 2.8$ & $7198 \pm 21$ & 7972 \\
\hline $6026 \mathrm{BC}$ & $74.8 \pm 2.3$ & $7171 \pm 17$ & 7975 \\
\hline $6036 \mathrm{BC}$ & $70.6 \pm 2.8$ & $7212 \pm 21$ & 7985 \\
\hline 6043 вС & $81.1 \pm 1.9$ & $7142 \pm 15$ & 7992 \\
\hline $6176 \mathrm{BC}$ & $82.5 \pm 1.8$ & $7259 \pm 13$ & 8125 \\
\hline 6186 BC & $80.4 \pm 2.7$ & $7285 \pm 20$ & 8135 \\
\hline $6196 \mathrm{BC}$ & $86.6 \pm 2.1$ & $7248 \pm 16$ & 8145 \\
\hline 6206 вC & $84.0 \pm 2.2$ & $7278 \pm 16$ & 8155 \\
\hline $6216 \mathrm{BC}$ & $82.4 \pm 3.6$ & $7299 \pm 27$ & 8165 \\
\hline $6226 \mathrm{BC}$ & $79.5 \pm 2.8$ & $7331 \pm 21$ & 8175 \\
\hline $6236 \mathrm{BC}$ & $67.1 \pm 3.7$ & $7433 \pm 28$ & 8185 \\
\hline 6246 ВС & $74.3 \pm 2.8$ & $7389 \pm 21$ & 8195 \\
\hline 6256 вC & $70.6 \pm 3.6$ & $7426 \pm 27$ & 8205 \\
\hline $6266 \mathrm{BC}$ & $73.7 \pm 2.9$ & $7412 \pm 22$ & 8215 \\
\hline $6276 \mathrm{BC}$ & $66.7 \pm 3.8$ & $7475 \pm 29$ & 8225 \\
\hline $6286 \mathrm{BC}$ & $79.1 \pm 3.0$ & $7392 \pm 23$ & 8235 \\
\hline $6296 \mathrm{BC}$ & $77.7 \pm 2.2$ & $7412 \pm 16$ & 8245 \\
\hline $6306 \mathrm{BC}$ & $74.9 \pm 2.0$ & $7442 \pm 15$ & 8255 \\
\hline $6316 \mathrm{BC}$ & $83.6 \pm 2.3$ & $7388 \pm 17$ & 8265 \\
\hline $6326 \mathrm{BC}$ & $84.4 \pm 2.2$ & $7391 \pm 16$ & 8275 \\
\hline 6336 BC & $85.0 \pm 2.8$ & $7397 \pm 21$ & 8285 \\
\hline 6346 BC & $83.4 \pm 2.0$ & $7418 \pm 15$ & 8295 \\
\hline 6396 вС & $80.4 \pm 3.5$ & $7489 \pm 26$ & 8345 \\
\hline 6406 BC & $79.9 \pm 2.1$ & $7501 \pm 15$ & 8355 \\
\hline 6416 BC & $81.5 \pm 2.0$ & $7500 \pm 15$ & 8365 \\
\hline $6426 \mathrm{BC}$ & $80.2 \pm 1.8$ & $7520 \pm 14$ & 8375 \\
\hline $6436 \mathrm{BC}$ & $75.6 \pm 3.0$ & $7564 \pm 22$ & 8385 \\
\hline 6446 BC & $75.2 \pm 2.0$ & $7576 \pm 16$ & 8395 \\
\hline 6456 BC & $75.2 \pm 2.1$ & $7586 \pm 16$ & 8405 \\
\hline $6466 \mathrm{BC}$ & $70.7 \pm 2.0$ & $7629 \pm 15$ & 8415 \\
\hline $6476 \mathrm{BC}$ & $68.7 \pm 1.7$ & $7655 \pm 13$ & 8425 \\
\hline $6486 \mathrm{BC}$ & $63.5 \pm 3.0$ & $7703 \pm 23$ & 8435 \\
\hline 6496 ВС & $70.1 \pm 3.1$ & $7663 \pm 24$ & 8445 \\
\hline 4) $6499 \mathrm{BC}$ & $68.3 \pm 2.3$ & $7679 \pm 17$ & 8448 \\
\hline
\end{tabular}

TABLE 1. Decadal Measurements (Continued)

\begin{tabular}{|c|c|c|c|c|}
\hline & I $\mathrm{AD} / \mathrm{BC}$ & $\Delta^{14} \mathrm{C}(\% o)$ & ${ }^{14} \mathrm{C}$ BP & cal BP \\
\hline & $6506 \mathrm{BC}$ & $71.6 \pm 2.9$ & $7662 \pm 22$ & 8455 \\
\hline (5) & $6514 \mathrm{BC}$ & $60.4 \pm 2.2$ & $7753 \pm 17$ & 8463 \\
\hline & $6516 \mathrm{BC}$ & $68.7 \pm 2.0$ & $7693 \pm 15$ & 8465 \\
\hline & 6526 BC & $70.5 \pm 2.0$ & $7690 \pm 16$ & 8475 \\
\hline & $6536 \mathrm{BC}$ & $65.5 \pm 2.8$ & $7736 \pm$ & 8485 \\
\hline (8) & $6538 \mathrm{BC}$ & $61.8 \pm 3.2$ & $7766 \pm 24$ & 8487 \\
\hline & $6546 \mathrm{BC}$ & $73.4 \pm 2.2$ & $7687 \pm 16$ & 8495 \\
\hline (8) & $6555 \mathrm{BC}$ & $75.8 \pm 2.2$ & $7677 \pm 16$ & 8504 \\
\hline & $6556 \mathrm{BC}$ & $73.1 \pm 2.1$ & $7699 \pm 16$ & 8505 \\
\hline & 6566 BC & $76.8 \pm 3.1$ & $7681 \pm 23$ & 8515 \\
\hline & $6576 \mathrm{BC}$ & $69.6 \pm 1.7$ & $7745 \pm 13$ & 8525 \\
\hline & $6586 \mathrm{BC}$ & $79.7 \pm 1.8$ & $7679 \pm 13$ & 8535 \\
\hline & $6596 \mathrm{BC}$ & $72.3 \pm 2.8$ & $7743 \pm 21$ & 8545 \\
\hline (4) & $6600 \mathrm{BC}$ & $68.2 \pm 3.2$ & $7778 \pm 24$ & 8549 \\
\hline & $6606 \mathrm{BC}$ & $70.4 \pm 1.9$ & $7767 \pm 15$ & 8555 \\
\hline & 6616 BC & $68.2 \pm 2.1$ & $7792 \pm 16$ & 8565 \\
\hline & $6626 \mathrm{BC}$ & $76.5 \pm 2.2$ & $7742 \pm 16$ & 8575 \\
\hline & 6629 BC & $65.5 \pm 2.8$ & $7826 \pm 21$ & 8578 \\
\hline & $6636 \mathrm{BC}$ & $74.9 \pm 2.1$ & $7763 \pm 16$ & 8585 \\
\hline & 6646 BC & $67.2 \pm 1.5$ & $7830 \pm 11$ & 8595 \\
\hline & 6656 ВС & $64.6 \pm 3.3$ & $7860 \pm 25$ & 8605 \\
\hline (6) & 6662 BC & $0.0 \pm 3.3$ & $7900 \pm 25$ & 8611 \\
\hline & 6666 BC & $3.0 \pm 3.3$ & $7844 \pm 25$ & 8615 \\
\hline & 6676 BC & $69.8 \pm 1.8$ & $7841 \pm 14$ & 8625 \\
\hline & $6686 \mathrm{BC}$ & $69.3 \pm 3.1$ & $7854 \pm 23$ & 8635 \\
\hline (11) & $6693 \mathrm{BC}$ & $62.7 \pm 3.1$ & $7910 \pm 24$ & 8642 \\
\hline & 6696 BC & $69.8 \pm 3.5$ & $7859 \pm 26$ & 8645 \\
\hline (5) & $6701 \mathrm{BC}$ & $60.5 \pm 3.0$ & $7934 \pm 23$ & 8650 \\
\hline & $6706 \mathrm{BC}$ & $64.4 \pm 3.1$ & $7910 \pm 23$ & 8655 \\
\hline & $6716 \mathrm{BC}$ & $64.2 \pm 2.3$ & $7921 \pm 18$ & 8665 \\
\hline (5) & $6721 \mathrm{BC}$ & $3.5 \pm 3.2$ & $7932 \pm 24$ & 8670 \\
\hline & 6726 BC & \pm 2.8 & & 8675 \\
\hline & $6736 \mathrm{BC}$ & $67.6 \pm 3.2$ & $7915 \pm 24$ & 8685 \\
\hline (5) & 6741 BC & $68.8 \pm 3.0$ & $7911 \pm 23$ & 8690 \\
\hline & 6746 BC & $72.6 \pm 1.9$ & $7886 \pm 14$ & 8695 \\
\hline & 6756 BC & 1.9 & \pm 15 & 8705 \\
\hline (5) & 6761 BC & $69.3 \pm 3.0$ & $7927 \pm 23$ & 8710 \\
\hline & $6766 \mathrm{BC}$ & $73.1 \pm 1.8$ & $7903 \pm 14$ & 8715 \\
\hline (3) & $6767 \mathrm{BC}$ & $67.6 \pm 3.3$ & $7945 \pm 25$ & 8716 \\
\hline & $6776 \mathrm{BC}$ & $71.4 \pm 3.1$ & $7925 \pm 23$ & 8725 \\
\hline & $6786 \mathrm{BC}$ & \pm 2.2 & \pm 16 & 8735 \\
\hline (3) & $6787 \mathrm{BC}$ & $70.3 \pm 3.4$ & $7944 \pm 26$ & \\
\hline & 6796 BC & $74.4 \pm 3.6$ & $7922 \pm 27$ & 8745 \\
\hline (3) & $6804 \mathrm{BC}$ & $73.2 \pm 3.4$ & $7939 \pm 26$ & 8753 \\
\hline & 6806 BC & $.7 \pm 3.0$ & $7922 \pm 22$ & 8755 \\
\hline & & & \pm 16 & 8765 \\
\hline & 6826 BC & $74.9 \pm 2.3$ & $7948 \pm 18$ & 8775 \\
\hline (4) & 6831 BC & $64.8 \pm 3.4$ & $8028 \pm 26$ & 8780 \\
\hline & $6836 \mathrm{BC}$ & $77.5 \pm 3.6$ & $7938 \pm 27$ & 8785 \\
\hline & 6846 вС & $.4 \pm 2.9$ & $8009 \pm 22$ & 8795 \\
\hline & & $68.3 \pm 3.4$ & $8023 \pm 26$ & 8802 \\
\hline & $6856 \mathrm{BC}$ & $80.4 \pm 3.6$ & $7936 \pm 27$ & 8805 \\
\hline & 6866 BC & $69.6 \pm 2.8$ & $8026 \pm 21$ & 8815 \\
\hline (5) & 6871 BC & $66.8 \pm 3.2$ & $8052 \pm 25$ & 8820 \\
\hline & & 2.1 & $7992 \pm 16$ & 8825 \\
\hline & 6886 BC & $81.6 \pm 3.0$ & $7956 \pm 22$ & 8835 \\
\hline & 6896 BC & $83.5 \pm 2.1$ & $7952 \pm 16$ & 8845 \\
\hline (5) & 6901 BC & $78.6 \pm 3.2$ & $7993 \pm 24$ & 8850 \\
\hline & 6906 BC & $86.8 \pm 3.0$ & $7937 \pm 22$ & 8855 \\
\hline & $6916 \mathrm{BC}$ & $88.3 \pm 3.1$ & & 8865 \\
\hline
\end{tabular}


TABLE 1. Decadal Measurements (Continued)

\begin{tabular}{|c|c|c|c|}
\hline cal AD/BC & $\Delta^{14} \mathrm{C}(\% \circ)$ & ${ }^{14} \mathrm{C} \mathrm{BP}$ & cal BP \\
\hline (5) $6921 \mathrm{BC}$ & $82.0 \pm 3.2$ & $7988 \pm 24$ & 8870 \\
\hline 6926 вС & $78.7 \pm 1.6$ & $8016 \pm 12$ & 8875 \\
\hline (11) 6933 вС & $86.3 \pm 4.8$ & $7967 \pm 36$ & 8882 \\
\hline $6936 \mathrm{BC}$ & $89.6 \pm 2.8$ & $7946 \pm 21$ & 8885 \\
\hline (6) $6940 \mathrm{BC}$ & $87.6 \pm 3.3$ & $7964 \pm 24$ & 8889 \\
\hline $6946 \mathrm{BC}$ & $93.1 \pm 2.9$ & $7930 \pm 21$ & 8895 \\
\hline $6956 \mathrm{BC}$ & $86.9 \pm 2.7$ & $7984 \pm 20$ & 8905 \\
\hline (5) 6961 вC & $88.8 \pm 3.5$ & $7976 \pm 26$ & 8910 \\
\hline 6963 BC & $85.9 \pm 3.3$ & $7999 \pm 24$ & 8912 \\
\hline $6966 \mathrm{BC}$ & $90.5 \pm 2.3$ & $7969 \pm 17$ & 8915 \\
\hline $6976 \mathrm{BC}$ & $97.6 \pm 3.7$ & $7925 \pm 27$ & 8925 \\
\hline 6986 BC & $91.7 \pm 2.3$ & $7978 \pm 17$ & 8935 \\
\hline 6996 ВC & $98.2 \pm 3.3$ & $7941 \pm 24$ & 8945 \\
\hline (5) $7001 \mathrm{BC}$ & $95.7 \pm 4.4$ & $7964 \pm 32$ & 8950 \\
\hline $7006 \mathrm{BC}$ & $94.6 \pm 1.9$ & $7976 \pm 14$ & 8955 \\
\hline (11) $7013 \mathrm{BC}$ & $93.8 \pm 1.8$ & $7989 \pm 13$ & 8962 \\
\hline $7016 \mathrm{BC}$ & $99.5 \pm 2.2$ & $7950 \pm 16$ & 8965 \\
\hline $7026 \mathrm{BC}$ & $96.8 \pm 2.3$ & $7981 \pm 17$ & 8975 \\
\hline (5) $7031 \mathrm{BC}$ & $88.3 \pm 3.3$ & $8048 \pm 24$ & 8980 \\
\hline $7036 \mathrm{BC}$ & $94.4 \pm 3.2$ & $8007 \pm 24$ & 8985 \\
\hline 7046 BC & $98.4 \pm 3.1$ & $7988 \pm 23$ & 8995 \\
\hline (5) $7051 \mathrm{BC}$ & $91.8 \pm 3.2$ & $8041 \pm 24$ & 9000 \\
\hline $7056 \mathrm{BC}$ & $98.6 \pm 3.1$ & $7996 \pm 22$ & 9005 \\
\hline (5) $7061 \mathrm{BC}$ & $83.4 \pm 3.2$ & $8113 \pm 24$ & 9010 \\
\hline $7066 \mathrm{BC}$ & $84.1 \pm 2.3$ & $8113 \pm 17$ & 9015 \\
\hline $7076 \mathrm{BC}$ & $86.7 \pm 2.1$ & $8103 \pm 16$ & 9025 \\
\hline $7086 \mathrm{BC}$ & $79.2 \pm 2.2$ & $8168 \pm 16$ & 9035 \\
\hline 7096 вС & $81.4 \pm 3.8$ & $8162 \pm 29$ & 9045 \\
\hline $7106 \mathrm{BC}$ & $90.3 \pm 2.9$ & $8105 \pm 21$ & 9055 \\
\hline (5) $7111 \mathrm{BC}$ & $78.7 \pm 3.2$ & $8196 \pm 24$ & 9060 \\
\hline $7116 \mathrm{BC}$ & $91.8 \pm 2.8$ & $8104 \pm 21$ & 9065 \\
\hline $7126 \mathrm{BC}$ & $86.9 \pm 4.0$ & $8150 \pm 30$ & 9075 \\
\hline $7136 \mathrm{BC}$ & $90.4 \pm 2.3$ & $8134 \pm 17$ & 9085 \\
\hline $7146 \mathrm{BC}$ & $84.1 \pm 2.1$ & \pm 16 & 9095 \\
\hline $6 \mathrm{BC}$ & $96.4 \pm 3.0$ & \pm 22 & 9105 \\
\hline 7166 BC & $90.9 \pm 2.2$ & $8159 \pm 16$ & 9115 \\
\hline $7176 \mathrm{BC}$ & $92.0 \pm 2.2$ & $8161 \pm 16$ & 9125 \\
\hline $7186 \mathrm{BC}$ & $83.8 \pm 2.1$ & $8232 \pm 16$ & 9135 \\
\hline (4) $7190 \mathrm{BC}$ & $88.5 \pm 3.3$ & & 9139 \\
\hline BC & $84.2 \pm 1.8$ & $8238 \pm 14$ & 9145 \\
\hline (2) $7200 \mathrm{BC}$ & $79.6 \pm 5.5$ & $8275 \pm 41$ & 9149 \\
\hline $7326 \mathrm{BC}$ & $96.7 \pm 3.4$ & $8272 \pm 25$ & 9275 \\
\hline $7336 \mathrm{BC}$ & $95.5 \pm 4.3$ & $1 \pm 32$ & 9285 \\
\hline 7346 BC & \pm 3.0 & & 9295 \\
\hline & $93.6 \pm 3.0$ & & 9305 \\
\hline $\mathrm{BC}$ & $97.7 \pm 3.1$ & $8315 \pm$ & 9327 \\
\hline 7388 вС & $89.5 \pm 2.7$ & $8385 \pm 20$ & 9337 \\
\hline 7398 BC & $104.0 \pm 3.3$ & 24 & 9347 \\
\hline 7408 BC & & & 9357 \\
\hline & $111.0 \pm 3.3$ & & 9367 \\
\hline 7428 BC & $97.4 \pm 3.7$ & $8366 \pm$ & 9377 \\
\hline 7438 вС & $106.3 \pm 3.5$ & $8311 \pm$ & 9387 \\
\hline 7448 вС & & & 9397 \\
\hline & $95.7 \pm 2.3$ & & 9407 \\
\hline 7468 вС & $108.6 \pm 3.1$ & $8324 \pm$ & 9417 \\
\hline 7478 вС & $109.3 \pm 3.0$ & $8328 \pm$ & 9427 \\
\hline 7488 вС & $101.9 \pm 3.1$ & & 9437 \\
\hline & $98.2 \pm 3.4$ & $8504 \pm$ & 9525 \\
\hline BC & $94.3 \pm 3.1$ & & 9535 \\
\hline $\mathrm{BC}$ & $85.9 \pm 3.3$ & & 9537 \\
\hline
\end{tabular}

TABLE 1. Decadal Measurements (Continued)

\begin{tabular}{|c|c|c|c|}
\hline cal $\mathrm{AD} / \mathrm{BC}$ & $\Delta^{14} \mathrm{C}(\% o)$ & ${ }^{14} \mathrm{C}$ BP & cal BP \\
\hline 7598 BC & $88.5 \pm 3.2$ & $8597 \pm 24$ & 9547 \\
\hline $7608 \mathrm{BC}$ & $81.0 \pm 3.0$ & $8663 \pm 22$ & 9557 \\
\hline $7618 \mathrm{BC}$ & $73.6 \pm 3.0$ & $8727 \pm 22$ & 9567 \\
\hline $7628 \mathrm{BC}$ & $88.7 \pm 3.2$ & $8625 \pm 24$ & 9577 \\
\hline $7638 \mathrm{BC}$ & $82.1 \pm 3.4$ & $8683 \pm 25$ & 9587 \\
\hline $7648 \mathrm{BC}$ & $88.9 \pm 3.4$ & $8643 \pm 25$ & 9597 \\
\hline $7658 \mathrm{BC}$ & $83.5 \pm 3.3$ & $8692 \pm 25$ & 9607 \\
\hline $7678 \mathrm{BC}$ & $97.4 \pm 4.6$ & $8609 \pm 34$ & 9627 \\
\hline $7688 \mathrm{BC}$ & $81.1 \pm 2.8$ & $8739 \pm 21$ & 9637 \\
\hline $7698 \mathrm{BC}$ & $86.0 \pm 3.4$ & $8712 \pm 25$ & 9647 \\
\hline $7708 \mathrm{BC}$ & $89.8 \pm 5.1$ & $8694 \pm 38$ & 9657 \\
\hline $7718 \mathrm{BC}$ & $86.6 \pm 4.2$ & $8728 \pm 31$ & 9667 \\
\hline $7728 \mathrm{BC}$ & $89.4 \pm 4.2$ & $8716 \pm 31$ & 9677 \\
\hline 7738 вС & $82.9 \pm 4.2$ & $8774 \pm 31$ & 9687 \\
\hline $7748 \mathrm{BC}$ & $97.4 \pm 4.5$ & $8677 \pm 33$ & 9697 \\
\hline $8007 \mathrm{BC}$ & $99.0 \pm 2.2$ & $8917 \pm 16$ & 9956 \\
\hline $8017 \mathrm{BC}^{*}$ & $103.7 \pm 3.1$ & $8893 \pm 23$ & 9966 \\
\hline 8027 ВC* $^{*}$ & $105.0 \pm 3.1$ & $8893 \pm 23$ & 9976 \\
\hline 8037 вс$^{*}$ & $107.3 \pm 4.4$ & $8886 \pm 32$ & 9986 \\
\hline 8047 вс* & $108.6 \pm 4.4$ & $8886 \pm 32$ & 9996 \\
\hline $8057 \mathrm{BC}$ & $114.6 \pm 3.1$ & $8853 \pm 23$ & 10006 \\
\hline $8067 \mathrm{BC}$ & $111.9 \pm 2.9$ & $8883 \pm 21$ & 10016 \\
\hline 8077 ВС & $114.8 \pm 3.2$ & $8871 \pm 23$ & 10026 \\
\hline $8087 \mathrm{BC}$ & $111.2 \pm 2.1$ & $8906 \pm 15$ & 10036 \\
\hline 8097 вС & $114.0 \pm 3.3$ & $8896 \pm 24$ & 10046 \\
\hline $8107 \mathrm{BC}$ & $122.9 \pm 3.1$ & $8842 \pm 22$ & 10056 \\
\hline $8117 \mathrm{BC}$ & $120.9 \pm 3.0$ & $8866 \pm 22$ & 10066 \\
\hline $8127 \mathrm{BC}$ & $116.4 \pm 3.7$ & $8908 \pm 27$ & 10076 \\
\hline $8137 \mathrm{BC}$ & $119.1 \pm 3.7$ & $8898 \pm 27$ & 10086 \\
\hline $8147 \mathrm{BC}$ & $128.4 \pm 3.3$ & $8841 \pm 24$ & 10096 \\
\hline $8157 \mathrm{BC}$ & $130.6 \pm 2.6$ & $8835 \pm 18$ & 10106 \\
\hline $8167 \mathrm{BC}$ & $126.5 \pm 3.3$ & $8874 \pm 24$ & 10116 \\
\hline $8177 \mathrm{BC}$ & $123.8 \pm 3.2$ & $8903 \pm 23$ & 10126 \\
\hline $8187 \mathrm{BC}$ & $132.1 \pm 3.3$ & $8854 \pm 23$ & 10136 \\
\hline $8197 \mathrm{BC}$ & $130.2 \pm 3.3$ & $8877 \pm 24$ & 10146 \\
\hline 8207 BC & $127.6 \pm 3.5$ & $8905 \pm 25$ & 10156 \\
\hline $8217 \mathrm{BC}$ & $122.4 \pm 3.4$ & \pm 24 & 10166 \\
\hline $27 \mathrm{BC}$ & $120.3 \pm 3.4$ & $8977 \pm 24$ & 10176 \\
\hline 8237 вC & $122.1 \pm 2.8$ & $8974 \pm 20$ & 10186 \\
\hline $8247 \mathrm{BC}$ & $117.7 \pm 2.8$ & $9015 \pm 20$ & 10196 \\
\hline 8257 BC & $124.1 \pm 2.5$ & \pm 18 & 1020 \\
\hline 8267 вС & $116.9 \pm 2.3$ & $9041 \pm 17$ & 10216 \\
\hline 8277 вС & $110.8 \pm 2.0$ & $9094 \pm 14$ & 10226 \\
\hline 8287 BC & $106.2 \pm 3.5$ & $9137 \pm 26$ & 10236 \\
\hline 8297 BC & $105.1 \pm 3.4$ & $9155 \pm 25$ & 10246 \\
\hline $8307 \mathrm{BC}$ & $104.4 \pm 2.3$ & \pm 17 & 10256 \\
\hline $7 \mathrm{BC}$ & $103.2 \pm 2.3$ & $9189 \pm 17$ & 10266 \\
\hline 8327 вС & $104.9 \pm 3.4$ & $9185 \pm 25$ & 10276 \\
\hline 8337 BC & $101.9 \pm 4.0$ & $9216 \pm 29$ & 10286 \\
\hline $8347 \mathrm{BC}$ & $98.9 \pm 2.0$ & $9247 \pm 15$ & 10296 \\
\hline 8357 ВС & $109.7 \pm 3.9$ & $9180 \pm 29$ & 10306 \\
\hline $67 \mathrm{BC}$ & $104.0 \pm 3.3$ & $9231 \pm 24$ & 10316 \\
\hline 8377 BC & $115.0 \pm 3.2$ & $9161 \pm 23$ & 10326 \\
\hline 8387 BC & $107.9 \pm 3.2$ & $9222 \pm 23$ & 10336 \\
\hline 8397 вС & & $9169 \pm 25$ & 10346 \\
\hline $407 \mathrm{BC}$ & $120.1 \pm 3.2$ & $9153 \pm 23$ & 10356 \\
\hline 8417 вС & $115.7 \pm 3.2$ & $9194 \pm 23$ & 10366 \\
\hline 8427 BC & $117.2 \pm 3.2$ & $9194 \pm 23$ & 10376 \\
\hline 8437 ВС & $116.4 \pm 3.4$ & & 10386 \\
\hline 8447 BC & $118.4 \pm 3.2$ & $9204 \pm 23$ & 10396 \\
\hline
\end{tabular}


TABLE 1. Decadal Measurements (Continued)

\begin{tabular}{|c|c|c|c|}
\hline cal $\mathrm{AD} / \mathrm{BC}$ & $\Delta^{14} \mathrm{C}(\% o)$ & ${ }^{14} \mathrm{C} B P$ & cal BP \\
\hline 8457 BC & $115.6 \pm 3.2$ & $9234 \pm 23$ & 10406 \\
\hline 8467 BC & $116.0 \pm 3.4$ & $9241 \pm 24$ & 10416 \\
\hline 8477 ВС & $116.8 \pm 3.2$ & $9245 \pm 23$ & 10426 \\
\hline 8487 BC & $109.0 \pm 2.4$ & $9311 \pm 17$ & 10436 \\
\hline 8497 вС & $121.8 \pm 3.1$ & $9229 \pm 22$ & 10446 \\
\hline 8507 BC & $122.9 \pm 3.4$ & $9230 \pm 24$ & 10456 \\
\hline 8517 BC & $123.6 \pm 3.2$ & $9235 \pm 23$ & 10466 \\
\hline $8527 \mathrm{BC}$ & $122.3 \pm 2.4$ & $9254 \pm 17$ & 10476 \\
\hline 8537 вC & $120.1 \pm 2.4$ & $9280 \pm 18$ & 10486 \\
\hline 8547 BC & $121.8 \pm 3.3$ & $9277 \pm 24$ & 10496 \\
\hline $8557 \mathrm{BC}$ & $119.9 \pm 3.1$ & $9300 \pm 22$ & 10506 \\
\hline $8567 \mathrm{BC}$ & $116.3 \pm 3.2$ & $9336 \pm 23$ & 10516 \\
\hline 8577 BC & $121.8 \pm 2.5$ & $9306 \pm 18$ & 10526 \\
\hline 8587 BC & $120.3 \pm 3.2$ & $9327 \pm 23$ & 10536 \\
\hline 8597 BC & $123.4 \pm 2.5$ & $9314 \pm 18$ & 10546 \\
\hline 8607 BC & $126.2 \pm 3.9$ & $9304 \pm 28$ & 10556 \\
\hline 8617 BC & $121.3 \pm 3.7$ & $9349 \pm 27$ & 10566 \\
\hline 8627 BC & $122.4 \pm 3.5$ & $9350 \pm 25$ & 10576 \\
\hline 8637 BC & $123.5 \pm 3.3$ & $9352 \pm 24$ & 10586 \\
\hline 8647 BC & $119.1 \pm 2.4$ & $9394 \pm 17$ & 10596 \\
\hline 8657 BC & $116.9 \pm 5.1$ & $9419 \pm 37$ & 10606 \\
\hline 8667 BC & $127.6 \pm 3.2$ & $9352 \pm 23$ & 10616 \\
\hline 8677 BC & $130.4 \pm 3.4$ & $9342 \pm 24$ & 10626 \\
\hline 8687 BC & $8 \pm 2.3$ & $9371 \pm 16$ & 10636 \\
\hline 8697 BC & $118.0 \pm 3.4$ & $9450 \pm 24$ & 10646 \\
\hline 8707 BC & $124.3 \pm 3.3$ & $9415 \pm 24$ & 10656 \\
\hline 8717 BC & $128.9 \pm 3.4$ & $9391 \pm 25$ & 10666 \\
\hline 8727 BC & $126.9 \pm 3.3$ & $9416 \pm 23$ & 10676 \\
\hline 8737 BC & $126.1 \pm 2.5$ & $9430 \pm 18$ & 10686 \\
\hline 8747 BC & $123.3 \pm 3.4$ & $9460 \pm 25$ & 10696 \\
\hline 8837 BC & $126.9 \pm 3.2$ & $9522 \pm 23$ & 10786 \\
\hline 8847 вС & $117.4 \pm 3.4$ & $9600 \pm 25$ & 10796 \\
\hline $57 \mathrm{BC}$ & \pm 3.3 & 95 & 10806 \\
\hline 8867 BC & $116.1 \pm 3.5$ & $9629 \pm 25$ & 10816 \\
\hline 8877 вС & $127.3 \pm 3.3$ & $9559 \pm 23$ & 10826 \\
\hline 8887 BC & $129.2 \pm 2.4$ & $9553 \pm 17$ & 10836 \\
\hline 8897 BC & $9 \pm 2.3$ & \pm 17 & 10846 \\
\hline 8907 BC & $126.2 \pm 3.8$ & $9595 \pm 27$ & 10856 \\
\hline 8917 BC & $121.8 \pm 3.5$ & $9636 \pm 25$ & 10866 \\
\hline 8927 BC & $126.5 \pm 3.5$ & $9613 \pm 25$ & 10876 \\
\hline 8937 BC & $124.3 \pm 3.5$ & $9638 \pm 25$ & 10886 \\
\hline 8947 BC & $8 \pm 3.5$ & & 10896 \\
\hline 8957 BC & $124.7 \pm 3.4$ & $9655 \pm 24$ & 10906 \\
\hline 8967 BC & $133.8 \pm 3.2$ & $9599 \pm 23$ & 10916 \\
\hline 8977 BC & $132.7 \pm 3.5$ & $9617 \pm 25$ & 10926 \\
\hline 8987 BC & $132.9 \pm 3.5$ & $9625 \pm 25$ & 10936 \\
\hline 8997 BC & $143.6 \pm 3.1$ & $9560 \pm 22$ & 10946 \\
\hline $9007 \mathrm{BC}$ & $144.4 \pm 2.5$ & $9564 \pm 17$ & 10956 \\
\hline 9017 BC & $149.1 \pm 2.7$ & $9541 \pm 19$ & 10966 \\
\hline $9067 \mathrm{BC}$ & $152.2 \pm 3.3$ & $9568 \pm 23$ & 11016 \\
\hline 9077 вС & $159.3 \pm 2.5$ & $9528 \pm 17$ & 11026 \\
\hline 9087 BC & $163.1 \pm 3.3$ & $9511 \pm 23$ & 11036 \\
\hline 9097 BC & $158.7 \pm 3.5$ & $9552 \pm 24$ & 11046 \\
\hline
\end{tabular}

TABLE 1. Decadal Measurements (Continued)

\begin{tabular}{|c|c|c|c|}
\hline cal $\mathrm{AD} / \mathrm{BC}$ & $\Delta^{14} \mathrm{C}(\% o)$ & ${ }^{14} \mathrm{C} B \mathrm{BP}$ & cal BP \\
\hline 9107 BC & $161.6 \pm 3.3$ & $9541 \pm 23$ & 11056 \\
\hline $9117 \mathrm{BC}$ & $157.8 \pm 3.5$ & $9577 \pm$ & 11066 \\
\hline $9127 \mathrm{BC}$ & $154.3 \pm 3.3$ & $9611 \pm 23$ & 11076 \\
\hline $9137 \mathrm{BC}$ & $156.7 \pm 3.5$ & $9604 \pm 24$ & 11086 \\
\hline $9147 \mathrm{BC}$ & $151.2 \pm 3.3$ & $9652 \pm 23$ & 11096 \\
\hline $9157 \mathrm{BC}$ & $157.1 \pm 2.4$ & $9622 \pm 17$ & 11106 \\
\hline 9172 вС & $153.5 \pm 3.2$ & $9660 \pm 23$ & 11121 \\
\hline 9182 вC & $158.3 \pm 3.6$ & $9637 \pm 25$ & 11131 \\
\hline 9192 вC & $157.9 \pm 3.4$ & $9649 \pm 24$ & 11141 \\
\hline $9202 \mathrm{BC}$ & $158.4 \pm 3.5$ & $9655 \pm 25$ & 11151 \\
\hline $9212 \mathrm{BC}$ & $154.8 \pm 3.4$ & $9690 \pm 24$ & 11161 \\
\hline $9222 \mathrm{BC}$ & $147.1 \pm 3.4$ & $9753 \pm 24$ & 11171 \\
\hline $9232 \mathrm{BC}$ & $151.2 \pm 3.3$ & $9735 \pm 23$ & 11181 \\
\hline $9268 \mathrm{BC}$ & $146.9 \pm 3.6$ & $9800 \pm 25$ & 11217 \\
\hline $9278 \mathrm{BC}$ & $143.1 \pm 3.4$ & $9836 \pm 24$ & 11227 \\
\hline 9288 BC & $141.5 \pm 3.6$ & $9857 \pm 25$ & 11237 \\
\hline $9292 \mathrm{BC}$ & $135.4 \pm 3.6$ & $9904 \pm 26$ & 11241 \\
\hline 9298 вС & $135.5 \pm 3.4$ & $9909 \pm 24$ & 11247 \\
\hline $9302 \mathrm{BC}$ & $142.6 \pm 2.5$ & $9863 \pm 17$ & 11251 \\
\hline 9308 BC & $137.3 \pm 2.1$ & $9906 \pm 15$ & 11257 \\
\hline $9312 \mathrm{BC}$ & $127.8 \pm 3.5$ & $9977 \pm 25$ & 11261 \\
\hline $9322 \mathrm{BC}$ & $137.4 \pm 3.6$ & $9920 \pm 26$ & 11271 \\
\hline $9368 \mathrm{BC}$ & $131.3 \pm 2.4$ & $10007 \pm 17$ & 11317 \\
\hline 9378 BC & $141.9 \pm 3.5$ & $9942 \pm 25$ & 11327 \\
\hline $9388 \mathrm{BC}$ & $9 \pm 3.5$ & $9959 \pm 24$ & 11337 \\
\hline 9398 BC & $134.8 \pm 2.3$ & $10011 \pm 17$ & 11347 \\
\hline $9408 \mathrm{BC}$ & $131.8 \pm 3.6$ & $10042 \pm 25$ & 11357 \\
\hline $9418 \mathrm{BC}$ & $132.2 \pm 3.5$ & $10049 \pm 25$ & 11367 \\
\hline $9428 \mathrm{BC}$ & $131.4 \pm 3.3$ & $10065 \pm 24$ & 11377 \\
\hline 9438 BC & $140.1 \pm 2.7$ & $10013 \pm 19$ & 11387 \\
\hline 9448 BC & $145.2 \pm 3.2$ & $9987 \pm 22$ & 11397 \\
\hline 9458 вС & $144.8 \pm 3.2$ & $9999 \pm 22$ & 11407 \\
\hline 9468 BC & $145.9 \pm 3.1$ & $10001 \pm 22$ & 11417 \\
\hline 9478 BC & \pm 4.0 & $6 \pm 28$ & 11427 \\
\hline 9488 BC & $146.4 \pm 3.6$ & $10017 \pm 25$ & 11437 \\
\hline $9498 \mathrm{BC}$ & $140.3 \pm 4.1$ & $10069 \pm 29$ & 11447 \\
\hline $9508 \mathrm{BC}$ & $142.0 \pm 3.2$ & $10068 \pm 22$ & 11457 \\
\hline $9518 \mathrm{BC}$ & 142 & $10072 \pm 20$ & 11467 \\
\hline $9528 \mathrm{BC}$ & $156.3 \pm 3.5$ & $9987 \pm 25$ & 11477 \\
\hline $9538 \mathrm{BC}$ & $152.0 \pm 3.5$ & $10027 \pm 25$ & 11487 \\
\hline 9548 вС & $147.7 \pm 3.4$ & $10066 \pm 24$ & 11497 \\
\hline $9558 \mathrm{BC}$ & $158.8 \pm 3.6$ & $9999 \pm 25$ & 11507 \\
\hline $9568 \mathrm{BC}$ & 3.5 & \pm 24 & 11517 \\
\hline $9578 \mathrm{BC}$ & $164.0 \pm 3.5$ & $9982 \pm 25$ & 11527 \\
\hline $9588 \mathrm{BC}$ & $165.4 \pm 3.5$ & $9982 \pm 24$ & 11537 \\
\hline $9598 \mathrm{BC}$ & $165.0 \pm 3.5$ & $9995 \pm 24$ & 11547 \\
\hline $9608 \mathrm{BC}$ & $157.7 \pm 3.4$ & $10055 \pm 23$ & 11557 \\
\hline 9618 BC & & $10066 \pm 19$ & 11567 \\
\hline $9628 \mathrm{BC}$ & $150.2 \pm 3.8$ & $10126 \pm 26$ & 11577 \\
\hline $9638 \mathrm{BC}$ & $153.8 \pm 3.8$ & $10111 \pm 27$ & 11587 \\
\hline 9648 BC & $157.8 \pm 2.2$ & $10093 \pm 16$ & 11597 \\
\hline & & $10122 \pm 24$ & 11607 \\
\hline 9668 BC & $157.9 \pm 3.4$ & $10112 \pm 23$ & 11617 \\
\hline
\end{tabular}


TABLE $2 .{ }^{14} \mathrm{C}$ age determinations made at the University of Washington Quaternary Isotope Lab (Seattle). The cal AD (or cal $\mathrm{BP}$ ) ages represent determinations on single-year wood sections from one or more North American trees, with the exception that from AD $1890-1914$ the ${ }^{14} \mathrm{C}$ ages were constructed from the average of single-year determinations on an Alaskan tree and 2- and 3-yr samples of a Pacific Northwest tree. For the latter tree the same ${ }^{14} \mathrm{C}$ age was used for each single year of the 2-3 yr sample, with the standard deviation in the age increased by 1.4 or 1.7 times. $\Delta^{14} \mathrm{C}$ was calculated as defined in Stuiver and Polach (1977). No error multiplier has been included in the standard deviations.

TABLE 2. Single-Year Data

\begin{tabular}{|c|c|c|c|}
\hline $\begin{array}{l}\text { cal } \\
\mathrm{AD}\end{array}$ & $\Delta^{14} \mathrm{C}(\%)$ & ${ }^{14} \mathrm{C} \mathrm{BP}$ & $\begin{array}{l}\text { cal } \\
\text { BP }\end{array}$ \\
\hline 1954 & $-22.5 \pm 2.7$ & $179 \pm 23$ & -4 \\
\hline 1953 & $-24.1 \pm 1.8$ & $193 \pm 15$ & -3 \\
\hline 1952 & $-25.8 \pm 1.6$ & $208 \pm 14$ & -2 \\
\hline 1951 & $-25.5 \pm 1.7$ & $207 \pm 14$ & -1 \\
\hline 1950 & $-25.8 \pm 1.7$ & $210 \pm 14$ & 0 \\
\hline 1949 & $-26.0 \pm 1.7$ & $213 \pm 14$ & 1 \\
\hline 1948 & $-22.1 \pm 1.8$ & $182 \pm 15$ & 2 \\
\hline 1947 & $-21.6 \pm 1.6$ & $178 \pm 13$ & 3 \\
\hline 1945 & $-22.4 \pm 1.9$ & $187 \pm 16$ & 5 \\
\hline 1944 & $-23.1 \pm 1.3$ & $193 \pm 10$ & 6 \\
\hline 1943 & $-24.3 \pm 1.2$ & $204 \pm 10$ & 7 \\
\hline 1942 & $-20.4 \pm 1.2$ & $174 \pm 10$ & 8 \\
\hline 1941 & $-19.9 \pm 1.9$ & $170 \pm 16$ & 9 \\
\hline 1940 & $-23.0 \pm 2.0$ & $197 \pm 16$ & 10 \\
\hline 1939 & $-20.1 \pm 1.8$ & $174 \pm 15$ & 11 \\
\hline 1938 & $-16.2 \pm 1.2$ & $143 \pm 10$ & 12 \\
\hline 1937 & $-17.2 \pm 1.6$ & $152 \pm 13$ & 13 \\
\hline 1936 & $-16.5 \pm 1.7$ & $147 \pm 14$ & 14 \\
\hline 1935 & $-16.7 \pm 1.9$ & $150 \pm 15$ & 15 \\
\hline 1934 & $-15.6 \pm 1.8$ & $142 \pm 15$ & 16 \\
\hline 1933 & $-18.6 \pm 1.8$ & $167 \pm 14$ & 17 \\
\hline 1932 & $-20.7 \pm 1.2$ & $186 \pm 10$ & 18 \\
\hline 1931 & $-16.6 \pm 1.8$ & $153 \pm 15$ & 19 \\
\hline 1930 & $-14.5 \pm 1.2$ & $137 \pm 10$ & 20 \\
\hline 1929 & $-18.2 \pm 1.0$ & $168 \pm 8$ & 21 \\
\hline 1928 & $-15.5 \pm 1.2$ & $147 \pm 10$ & 22 \\
\hline 1927 & $-15.7 \pm 1.1$ & $149 \pm 9$ & 23 \\
\hline 1926 & $-14.7 \pm 1.2$ & $143 \pm$ & 24 \\
\hline 1925 & $-12.3 \pm 1.2$ & $124 \pm 9$ & 25 \\
\hline 1924 & $-11.4 \pm 1.2$ & $117 \pm 10$ & 26 \\
\hline 1923 & $-14.1 \pm 1.0$ & $140 \pm 8$ & 27 \\
\hline 1922 & $-12.2 \pm 1.2$ & $126 \pm 10$ & 28 \\
\hline 1921 & $-12.9 \pm 1.2$ & $133 \pm 10$ & 29 \\
\hline 1920 & $-14.1 \pm 1.3$ & $144 \pm 10$ & 30 \\
\hline 1919 & $-11.1 \pm 1.2$ & $120 \pm 10$ & 31 \\
\hline 1918 & $-11.3 \pm 1.0$ & $122 \pm 8$ & 32 \\
\hline 1917 & $-9.7 \pm 1.0$ & $110 \pm 8$ & 33 \\
\hline 1916 & $-11.0 \pm 1.2$ & $122 \pm 10$ & 34 \\
\hline & $-6.3 \pm 1.7$ & $85 \pm 14$ & 35 \\
\hline & $-7.1 \pm 1.0$ & $92 \pm 8$ & 36 \\
\hline & $-7.3 \pm 0.9$ & $95 \pm 7$ & 37 \\
\hline
\end{tabular}

TABLE 2. Single-Year Data (Continued)

\begin{tabular}{crrr} 
cal & & & cal \\
$\mathrm{AD}$ & $\Delta^{14} \mathrm{C}(\% \circ)$ & \multicolumn{1}{c}{${ }^{14} \mathrm{C} \mathrm{BP}$} & $\mathrm{BP}$ \\
\hline 1912 & $-8.1 \pm 1.1$ & $101 \pm 9$ & 38 \\
1911 & $-8.3 \pm 1.2$ & $105 \pm 10$ & 39 \\
1910 & $-7.5 \pm 1.2$ & $99 \pm 10$ & 40 \\
1909 & $-6.5 \pm 1.3$ & $92 \pm 11$ & 41 \\
1908 & $-8.4 \pm 1.4$ & $108 \pm 12$ & 42 \\
1907 & $-6.2 \pm 1.3$ & $92 \pm 10$ & 43 \\
1906 & $-4.4 \pm 1.2$ & $78 \pm 10$ & 44 \\
1905 & $-5.5 \pm 1.4$ & $88 \pm 11$ & 45 \\
1904 & $-4.3 \pm 1.4$ & $79 \pm 11$ & 46 \\
1903 & $-4.2 \pm 1.3$ & $80 \pm 11$ & 47 \\
1902 & $-2.9 \pm 1.1$ & $70 \pm 9$ & 48 \\
1901 & $1.2 \pm 1.1$ & $38 \pm 9$ & 49 \\
1900 & $-2.9 \pm 1.5$ & $72 \pm 12$ & 50 \\
1899 & $-4.5 \pm 1.3$ & $86 \pm 11$ & 51 \\
1898 & $-3.4 \pm 1.5$ & $78 \pm 12$ & 52 \\
1897 & $-3.4 \pm 1.4$ & $79 \pm 11$ & 53 \\
1896 & $-1.3 \pm 1.2$ & $63 \pm 10$ & 54 \\
1895 & $-2.1 \pm 1.2$ & $71 \pm 10$ & 55 \\
1894 & $-2.2 \pm 1.2$ & $72 \pm 10$ & 56 \\
1893 & $-2.2 \pm 1.3$ & $73 \pm 10$ & 57 \\
1892 & $-4.4 \pm 1.3$ & $92 \pm 10$ & 58 \\
1891 & $-3.2 \pm 1.4$ & $83 \pm 11$ & 59 \\
1890 & $-4.3 \pm 1.4$ & $93 \pm 11$ & 60 \\
1889 & $-5.4 \pm 1.1$ & $103 \pm 9$ & 61 \\
1888 & $-6.5 \pm 1.8$ & $113 \pm 15$ & 62 \\
1887 & $-6.6 \pm 1.1$ & $115 \pm 9$ & 63 \\
1886 & $-6.1 \pm 1.1$ & $111 \pm 9$ & 64 \\
1885 & $-4.5 \pm 1.2$ & $100 \pm 9$ & 65 \\
1884 & $-3.3 \pm 1.7$ & $91 \pm 14$ & 66 \\
1883 & $-2.7 \pm 1.2$ & $87 \pm 10$ & 67 \\
1882 & $-1.5 \pm 1.2$ & $78 \pm 9$ & 68 \\
1881 & $-4.9 \pm 1.6$ & $107 \pm 13$ & 69 \\
1880 & $-2.7 \pm 1.6$ & $90 \pm 13$ & 70 \\
1879 & $-5.1 \pm 1.5$ & $110 \pm 12$ & 71 \\
1878 & $-5.9 \pm 1.1$ & $118 \pm 9$ & 72 \\
1877 & $-4.9 \pm 1.5$ & $110 \pm 12$ & 73 \\
1876 & $-7.4 \pm 1.7$ & $132 \pm 14$ & 74 \\
1875 & $-5.0 \pm 1.1$ & $113 \pm 9$ & 75 \\
1874 & $-6.1 \pm 1.8$ & $123 \pm 14$ & 76 \\
1873 & $-6.2 \pm 1.2$ & $124 \pm 10$ & 77 \\
1872 & $-4.7 \pm 1.1$ & $114 \pm 9$ & 78
\end{tabular}


TABLE 2. Single-Year Data (Continued)

\begin{tabular}{crrr}
\hline cal & & & cal \\
AD & \multicolumn{1}{c}{$\Delta^{14} \mathrm{C}(\%)$} & \multicolumn{1}{c}{$14 \mathrm{C} \mathrm{BP}$} & BP \\
\hline 1871 & $-4.6 \pm 1.4$ & $114 \pm 11$ & 79 \\
1870 & $-4.8 \pm 1.3$ & $116 \pm 11$ & 80 \\
1869 & $-4.3 \pm 1.3$ & $114 \pm 10$ & 81 \\
1868 & $-4.4 \pm 1.3$ & $115 \pm 11$ & 82 \\
1867 & $-3.3 \pm 1.0$ & $108 \pm 8$ & 83 \\
1866 & $-5.0 \pm 1.8$ & $122 \pm 15$ & 84 \\
1865 & $-3.1 \pm 1.5$ & $107 \pm 12$ & 85 \\
1864 & $-5.6 \pm 1.6$ & $129 \pm 13$ & 86 \\
1863 & $-5.8 \pm 1.7$ & $131 \pm 14$ & 87 \\
1862 & $-7.2 \pm 2.0$ & $144 \pm 16$ & 88 \\
1861 & $-3.0 \pm 1.7$ & $110 \pm 13$ & 89 \\
1860 & $-4.4 \pm 1.7$ & $123 \pm 13$ & 90 \\
1859 & $-3.1 \pm 1.8$ & $113 \pm 15$ & 91 \\
1858 & $-2.1 \pm 1.9$ & $106 \pm 15$ & 92 \\
1857 & $-5.0 \pm 1.6$ & $131 \pm 13$ & 93 \\
1856 & $-4.0 \pm 1.2$ & $124 \pm 10$ & 94 \\
1855 & $-4.0 \pm 1.6$ & $125 \pm 13$ & 95 \\
1854 & $-4.1 \pm 2.6$ & $126 \pm 21$ & 96 \\
1853 & $-3.6 \pm 1.3$ & $123 \pm 11$ & 97 \\
1852 & $0.1 \pm 2.5$ & $95 \pm 20$ & 98 \\
1851 & $-2.5 \pm 2.5$ & $116 \pm 20$ & 99 \\
1850 & $-1.4 \pm 2.5$ & $109 \pm 20$ & 100 \\
1849 & $-0.5 \pm 1.5$ & $99 \pm 15$ & 101 \\
1848 & $-1.9 \pm 1.8$ & $114 \pm 15$ & 102 \\
1847 & $-0.7 \pm 1.7$ & $106 \pm 14$ & 103 \\
1846 & $-0.4 \pm 1.7$ & $104 \pm 14$ & 104 \\
1845 & $-2.5 \pm 2.5$ & $122 \pm 20$ & 105 \\
1844 & $-0.8 \pm 1.2$ & $109 \pm 10$ & 106 \\
1843 & $-2.0 \pm 1.0$ & $120 \pm 8$ & 107 \\
1842 & $-1.3 \pm 1.4$ & $116 \pm 11$ & 108 \\
1841 & $-4.7 \pm 1.7$ & $143 \pm 14$ & 109 \\
1840 & $-1.0 \pm 1.7$ & $115 \pm 14$ & 110 \\
1839 & $-1.2 \pm 1.7$ & $117 \pm 14$ & 111 \\
1838 & $-2.5 \pm 0.9$ & $129 \pm 8$ & 112 \\
1837 & $-3.4 \pm 1.2$ & $137 \pm 10$ & 113 \\
1836 & $0.2 \pm 1.2$ & $109 \pm 10$ & 114 \\
1835 & $0.0 \pm 1.6$ & $112 \pm 13$ & 115 \\
1834 & $-0.1 \pm 1.9$ & $114 \pm 16$ & 116 \\
1833 & $0.5 \pm 1.4$ & $110 \pm 11$ & 117 \\
1832 & $2.2 \pm 1.4$ & $97 \pm 11$ & 118 \\
1831 & $1.4 \pm 1.2$ & $104 \pm 10$ & 119 \\
1830 & $3.8 \pm 1.6$ & $86 \pm 13$ & 120 \\
1829 & $5.2 \pm 1.6$ & $76 \pm 13$ & 121 \\
1828 & $2.8 \pm 1.0$ & $96 \pm 8$ & 122 \\
1827 & $4.4 \pm 1.2$ & $84 \pm 9$ & 123 \\
1826 & $2.2 \pm 1.6$ & $103 \pm 13$ & 124 \\
1825 & $1.9 \pm 1.6$ & $107 \pm 13$ & 125 \\
1824 & $1.0 \pm 1.2$ & $114 \pm 10$ & 126 \\
1823 & $3.0 \pm 1.7$ & $100 \pm 14$ & 127 \\
1822 & $0.3 \pm 1.2$ & $122 \pm 10$ & 128 \\
1821 & $3.6 \pm 1.0$ & $96 \pm 8$ & 129 \\
1820 & $3.4 \pm 1.0$ & $99 \pm 8$ & 130 \\
& $-1.4 \pm 2.0$ & $139 \pm 16$ & 131
\end{tabular}

TABLE 2. Single-Year Data (Continued)

\begin{tabular}{crcc}
\hline cal & & & cal \\
$\mathrm{AD}$ & $\Delta^{14} \mathrm{C}(\%)$ & $14 \mathrm{C} \mathrm{BP}$ & $\mathrm{BP}$ \\
\hline 1818 & $6.1 \pm 1.3$ & $79 \pm 10$ & 132 \\
1817 & $6.2 \pm 1.9$ & $80 \pm 16$ & 133 \\
1816 & $5.8 \pm 1.8$ & $84 \pm 14$ & 134 \\
1815 & $1.7 \pm 1.8$ & $118 \pm 14$ & 135 \\
1814 & $0.8 \pm 2.0$ & $126 \pm 16$ & 136 \\
1813 & $1.5 \pm 1.2$ & $121 \pm 10$ & 137 \\
1812 & $2.5 \pm 2.0$ & $114 \pm 16$ & 138 \\
1811 & $1.6 \pm 2.0$ & $122 \pm 16$ & 139 \\
1810 & $2.1 \pm 1.9$ & $119 \pm 15$ & 140 \\
1809 & $-0.1 \pm 1.8$ & $138 \pm 15$ & 141 \\
1808 & $-5.5 \pm 1.8$ & $183 \pm 14$ & 142 \\
1807 & $-1.4 \pm 1.3$ & $150 \pm 11$ & 143 \\
1806 & $-3.7 \pm 1.8$ & $170 \pm 14$ & 144 \\
1805 & $-1.6 \pm 1.7$ & $154 \pm 14$ & 145 \\
1804 & $-1.5 \pm 1.7$ & $154 \pm 14$ & 146 \\
1803 & $-3.4 \pm 1.7$ & $170 \pm 14$ & 147 \\
1802 & $-2.5 \pm 1.3$ & $164 \pm 10$ & 148 \\
1801 & $-5.0 \pm 1.2$ & $185 \pm 10$ & 149 \\
1800 & $-0.1 \pm 1.7$ & $147 \pm 14$ & 150 \\
1799 & $-1.0 \pm 1.7$ & $155 \pm 14$ & 151 \\
1798 & $-4.1 \pm 1.2$ & $181 \pm 10$ & 152 \\
1797 & $-5.0 \pm 1.7$ & $189 \pm 14$ & 153 \\
1796 & $-9.3 \pm 1.8$ & $225 \pm 14$ & 154 \\
1795 & $-9.7 \pm 2.0$ & $229 \pm 16$ & 155 \\
1794 & $-9.4 \pm 1.7$ & $228 \pm 14$ & 156 \\
1793 & $-7.8 \pm 1.7$ & $215 \pm 14$ & 157 \\
1792 & $-10.7 \pm 1.8$ & $240 \pm 15$ & 158 \\
1791 & $-7.1 \pm 1.8$ & $212 \pm 15$ & 159 \\
1790 & $-7.3 \pm 1.7$ & $214 \pm 14$ & 160 \\
1789 & $-6.6 \pm 1.8$ & $209 \pm 14$ & 161 \\
1788 & $-10.2 \pm 1.8$ & $240 \pm 15$ & 162 \\
1787 & $-8.2 \pm 1.7$ & $224 \pm 14$ & 163 \\
1786 & $-7.8 \pm 2.3$ & $222 \pm 19$ & 164 \\
1785 & $-7.2 \pm 1.4$ & $219 \pm 12$ & 165 \\
1784 & $-7.7 \pm 1.7$ & $224 \pm 14$ & 166 \\
1781 & $-5.1 \pm 1.2$ & $205 \pm 10$ & 169 \\
1780 & $-1.4 \pm 1.2$ & $176 \pm 10$ & 170 \\
1779 & $-0.4 \pm 1.7$ & $170 \pm 14$ & 171 \\
1778 & $0.2 \pm 1.7$ & $165 \pm 14$ & 172 \\
1777 & $1.4 \pm 1.3$ & $157 \pm 10$ & 173 \\
1776 & $2.5 \pm 1.2$ & $149 \pm 10$ & 174 \\
1775 & $1.1 \pm 1.7$ & $162 \pm 14$ & 175 \\
1774 & $-1.3 \pm 1.7$ & $181 \pm 13$ & 176 \\
1773 & $1.4 \pm 1.2$ & $161 \pm 10$ & 177 \\
1772 & $-0.1 \pm 1.3$ & $174 \pm 11$ & 178 \\
1771 & $1.3 \pm 1.7$ & $164 \pm 14$ & 179 \\
1770 & $0.0 \pm 1.7$ & $175 \pm 14$ & 180 \\
1769 & $0.1 \pm 1.7$ & $175 \pm 14$ & 181 \\
1768 & $-0.8 \pm 1.7$ & $183 \pm 14$ & 182 \\
1767 & $-0.6 \pm 1.7$ & $183 \pm 14$ & 183 \\
1766 & $1.5 \pm 1.7$ & $167 \pm 14$ & 184 \\
1765 & $2.1 \pm 1.7$ & $163 \pm 14$ & 185 \\
1764 & $2.9 \pm 1.7$ & $157 \pm 14$ & 186
\end{tabular}


TABLE 2. Single-Year Data (Continued)

\begin{tabular}{rrrr} 
cal & & & cal \\
AD & $\Delta^{14} \mathrm{C}(\%)$ & $14 \mathrm{C} \mathrm{BP}$ & BP \\
\hline 1763 & $-0.7 \pm 1.7$ & $187 \pm 13$ & 187 \\
1762 & $2.6 \pm 1.7$ & $162 \pm 14$ & 188 \\
1761 & $4.6 \pm 1.2$ & $147 \pm 10$ & 189 \\
1760 & $4.6 \pm 1.3$ & $148 \pm 10$ & 190 \\
1759 & $5.3 \pm 1.3$ & $143 \pm 10$ & 191 \\
1758 & $6.0 \pm 1.0$ & $138 \pm 8$ & 192 \\
1757 & $4.3 \pm 1.3$ & $153 \pm 11$ & 193 \\
1756 & $3.6 \pm 1.1$ & $160 \pm 9$ & 194 \\
1755 & $3.8 \pm 1.2$ & $159 \pm 10$ & 195 \\
1754 & $4.5 \pm 1.8$ & $155 \pm 14$ & 196 \\
1753 & $6.0 \pm 1.1$ & $143 \pm 9$ & 197 \\
1752 & $4.7 \pm 1.7$ & $155 \pm 14$ & 198 \\
1751 & $2.5 \pm 1.3$ & $174 \pm 11$ & 199 \\
1750 & $5.1 \pm 1.2$ & $154 \pm 10$ & 200 \\
1748 & $4.5 \pm 1.7$ & $160 \pm 14$ & 202 \\
1747 & $6.9 \pm 1.7$ & $142 \pm 14$ & 203 \\
1746 & $7.4 \pm 1.2$ & $139 \pm 10$ & 204 \\
1745 & $4.6 \pm 1.3$ & $162 \pm 10$ & 205 \\
1744 & $2.8 \pm 1.7$ & $178 \pm 14$ & 206 \\
1743 & $5.0 \pm 1.1$ & $161 \pm 9$ & 207 \\
1742 & $5.5 \pm 1.8$ & $158 \pm 14$ & 208 \\
1741 & $1.1 \pm 1.3$ & $195 \pm 10$ & 209 \\
1740 & $5.6 \pm 1.7$ & $159 \pm 14$ & 210 \\
1739 & $6.4 \pm 1.3$ & $154 \pm 10$ & 211 \\
1738 & $7.2 \pm 1.7$ & $148 \pm 14$ & 212 \\
1737 & $7.3 \pm 2.0$ & $149 \pm 16$ & 213 \\
1736 & $7.4 \pm 2.0$ & $149 \pm 16$ & 214 \\
1735 & $7.3 \pm 1.8$ & $150 \pm 15$ & 215 \\
1734 & $8.9 \pm 2.3$ & $139 \pm 18$ & 216 \\
1733 & $8.9 \pm 1.8$ & $140 \pm 15$ & 217 \\
1732 & $7.1 \pm 1.4$ & $155 \pm 12$ & 218 \\
1731 & $5.6 \pm 1.4$ & $168 \pm 11$ & 219 \\
1730 & $10.6 \pm 1.8$ & $129 \pm 15$ & 220 \\
1729 & $12.8 \pm 1.8$ & $113 \pm 14$ & 221 \\
1728 & $11.6 \pm 1.8$ & $123 \pm 15$ & 222 \\
1727 & $14.2 \pm 1.8$ & $103 \pm 15$ & 223 \\
1726 & $18.0 \pm 1.5$ & $75 \pm 12$ & 224 \\
1725 & $13.1 \pm 1.1$ & $114 \pm 9$ & 225 \\
1724 & $12.8 \pm 1.0$ & $117 \pm 8$ & 226 \\
1723 & $13.5 \pm 1.3$ & $113 \pm 10$ & 227 \\
1722 & $12.7 \pm 1.2$ & $120 \pm 9$ & 228 \\
1721 & $13.7 \pm 1.2$ & $114 \pm 10$ & 229 \\
1720 & $14.8 \pm 1.0$ & $105 \pm 8$ & 230 \\
1719 & $16.3 \pm 1.0$ & $94 \pm 8$ & 231 \\
1718 & $17.0 \pm 0.8$ & $90 \pm 7$ & 232 \\
1717 & $15.8 \pm 1.0$ & $101 \pm 8$ & 233 \\
1716 & $17.5 \pm 1.3$ & $88 \pm 10$ & 234 \\
1715 & $16.7 \pm 1.2$ & $95 \pm 10$ & 235 \\
1714 & $17.9 \pm 1.2$ & $87 \pm 10$ & 236 \\
1713 & $17.6 \pm 1.2$ & $90 \pm 10$ & 237 \\
1712 & $13.9 \pm 1.2$ & $121 \pm 10$ & 238 \\
1711 & $18.5 \pm 0.9$ & $85 \pm 7$ & 239 \\
1710 & $17.5 \pm 1.0$ & $94 \pm 8$ & 240
\end{tabular}

TABLE 2. Single-Year Data (Continued)

\begin{tabular}{|c|c|c|c|}
\hline $\begin{array}{l}\text { cal } \\
\mathrm{AD}\end{array}$ & $\Delta^{14} \mathrm{C}(\% \circ)$ & ${ }^{14} \mathrm{C} \mathrm{BP}$ & $\begin{array}{l}\text { cal } \\
\text { BP }\end{array}$ \\
\hline 1709 & $16.9 \pm 1.3$ & $100 \pm 10$ & 241 \\
\hline 1708 & $15.4 \pm 1.2$ & $113 \pm 9$ & 242 \\
\hline 1707 & $15.0 \pm 0.8$ & $116 \pm 7$ & 243 \\
\hline 1706 & $14.4 \pm 1.3$ & $122 \pm 10$ & 244 \\
\hline 1705 & $16.5 \pm 1.3$ & $107 \pm 10$ & 245 \\
\hline 1704 & $19.1 \pm 0.9$ & $87 \pm 7$ & 246 \\
\hline 1703 & $17.7 \pm 0.9$ & $99 \pm$ & 247 \\
\hline 1702 & $15.2 \pm 0.8$ & $120 \pm$ & 248 \\
\hline 1701 & $19.0 \pm 1.0$ & $91 \pm$ & 249 \\
\hline 1700 & $17.5 \pm 0.9$ & $104 \pm$ & 250 \\
\hline 1699 & $18.0 \pm 1.1$ & $101 \pm$ & 251 \\
\hline 1698 & $17.4 \pm 1.1$ & $107 \pm$ & 252 \\
\hline 1697 & $18.2 \pm 1.1$ & $101 \pm$ & 253 \\
\hline 1696 & $16.6 \pm 1.0$ & $115 \pm$ & 254 \\
\hline 1695 & $15.8 \pm 0.8$ & $122 \pm$ & 255 \\
\hline 1694 & $16.3 \pm 0.9$ & $119 \pm$ & 256 \\
\hline 1693 & $15.7 \pm 1.0$ & $125 \pm$ & 257 \\
\hline 1692 & $16.3 \pm 1.0$ & $121 \pm$ & 258 \\
\hline 1691 & $14.7 \pm 1.0$ & $134 \pm$ & 259 \\
\hline 1690 & $13.9 \pm 0.9$ & $142 \pm$ & 260 \\
\hline 1689 & $15.7 \pm 1.3$ & $128 \pm 11$ & 261 \\
\hline 1688 & $16.6 \pm 1.3$ & $122 \pm 10$ & 262 \\
\hline 1687 & $14.1 \pm 1.1$ & $143 \pm 9$ & 263 \\
\hline 1686 & $17.6 \pm 0.9$ & $116 \pm 8$ & 264 \\
\hline 1685 & $13.5 \pm 2.4$ & $150 \pm 19$ & 265 \\
\hline 1684 & $13.2 \pm 1.8$ & $154 \pm 14$ & 266 \\
\hline 1683 & $11.5 \pm 1.8$ & $167 \pm 14$ & 267 \\
\hline 1682 & $12.2 \pm 1.9$ & $163 \pm 15$ & 268 \\
\hline 1681 & $12.0 \pm 1.8$ & $166 \pm 14$ & 269 \\
\hline 1680 & $12.9 \pm 1.7$ & $159 \pm 13$ & 270 \\
\hline 1678 & $8.5 \pm 1.8$ & $197 \pm 14$ & 272 \\
\hline 1677 & $9.9 \pm 1.2$ & $186 \pm 9$ & 273 \\
\hline 1676 & $11.3 \pm 1.2$ & $176 \pm 10$ & 274 \\
\hline 1675 & $12.0 \pm 1.7$ & $172 \pm 14$ & 275 \\
\hline 1674 & $9.3 \pm 1.4$ & $194 \pm 11$ & 276 \\
\hline 1673 & $15.4 \pm 1.3$ & $147 \pm 10$ & 277 \\
\hline 1672 & $10.9 \pm 1.8$ & $183 \pm 14$ & 278 \\
\hline 1671 & $15.1 \pm 1.3$ & $151 \pm 11$ & 279 \\
\hline 1670 & $9.8 \pm 1.8$ & $194 \pm 14$ & 280 \\
\hline 1669 & $11.2 \pm 1.9$ & $184 \pm 15$ & 281 \\
\hline 1668 & $12.0 \pm 2.0$ & $179 \pm 16$ & 282 \\
\hline 1667 & $10.2 \pm 1.9$ & $194 \pm 15$ & 283 \\
\hline 1666 & $8.3 \pm 1.8$ & $209 \pm 14$ & 284 \\
\hline 1665 & $6.7 \pm 1.4$ & $223 \pm 11$ & 285 \\
\hline 1664 & $6.1 \pm 1.1$ & $229 \pm 9$ & 286 \\
\hline 1663 & $8.7 \pm 1.9$ & $209 \pm 15$ & 287 \\
\hline 1662 & $7.5 \pm 1.8$ & $220 \pm 15$ & 288 \\
\hline 1661 & $9.0 \pm 1.9$ & $209 \pm 15$ & 289 \\
\hline 1660 & $3.9 \pm 1.3$ & $250 \pm 10$ & 290 \\
\hline 1659 & $6.2 \pm 1.9$ & $233 \pm 16$ & 291 \\
\hline 1658 & $9.4 \pm 1.9$ & $209 \pm 15$ & 292 \\
\hline & $4.4 \pm 1.5$ & $249 \pm 12$ & 293 \\
\hline 165 & $4.6 \pm 1.6$ & $249 \pm 13$ & 294 \\
\hline
\end{tabular}


TABLE 2. Single-Year Data (Continued)

\begin{tabular}{crcc}
\hline cal & & & cal \\
AD & \multicolumn{1}{c}{$\Delta^{14} \mathrm{C}(\%)$} & $14 \mathrm{C} \mathrm{BP}$ & BP \\
\hline 1655 & $6.3 \pm 1.6$ & $236 \pm 13$ & 295 \\
1654 & $7.1 \pm 1.3$ & $231 \pm 10$ & 296 \\
1653 & $4.0 \pm 1.9$ & $256 \pm 15$ & 297 \\
1651 & $4.9 \pm 1.9$ & $251 \pm 15$ & 299 \\
1650 & $5.7 \pm 1.3$ & $246 \pm 10$ & 300 \\
1649 & $4.0 \pm 1.3$ & $260 \pm 10$ & 301 \\
1648 & $3.3 \pm 1.8$ & $267 \pm 14$ & 302 \\
1647 & $4.0 \pm 1.9$ & $263 \pm 16$ & 303 \\
1646 & $2.6 \pm 1.8$ & $275 \pm 14$ & 304 \\
1645 & $2.2 \pm 1.7$ & $279 \pm 14$ & 305 \\
1644 & $2.2 \pm 1.0$ & $280 \pm 8$ & 306 \\
1643 & $3.8 \pm 1.8$ & $268 \pm 15$ & 307 \\
1642 & $1.5 \pm 1.8$ & $288 \pm 14$ & 308 \\
1641 & $2.1 \pm 2.2$ & $283 \pm 17$ & 309 \\
1640 & $-2.5 \pm 1.3$ & $321 \pm 11$ & 310 \\
1639 & $0.6 \pm 1.1$ & $298 \pm 9$ & 311 \\
1638 & $-2.6 \pm 1.7$ & $324 \pm 14$ & 312 \\
1637 & $0.6 \pm 2.2$ & $299 \pm 17$ & 313 \\
1636 & $1.5 \pm 1.3$ & $293 \pm 10$ & 314 \\
1635 & $1.3 \pm 1.2$ & $295 \pm 10$ & 315 \\
1634 & $-3.4 \pm 1.8$ & $334 \pm 15$ & 316 \\
1633 & $-0.2 \pm 1.8$ & $310 \pm 15$ & 317 \\
1632 & $-3.6 \pm 1.8$ & $338 \pm 15$ & 318 \\
1631 & $-3.3 \pm 1.8$ & $337 \pm 15$ & 319 \\
1630 & $-4.0 \pm 1.8$ & $343 \pm 15$ & 320 \\
1629 & $-1.0 \pm 1.2$ & $319 \pm 10$ & 321 \\
1628 & $-4.7 \pm 1.8$ & $351 \pm 15$ & 322 \\
1627 & $-0.4 \pm 1.8$ & $317 \pm 15$ & 323 \\
1626 & $-6.4 \pm 1.8$ & $366 \pm 15$ & 324 \\
1625 & $-0.8 \pm 1.1$ & $322 \pm 9$ & 325 \\
1624 & $-4.0 \pm 1.4$ & $349 \pm 11$ & 326 \\
1623 & $2.0 \pm 2.0$ & $302 \pm 16$ & 327 \\
1622 & $-2.6 \pm 1.3$ & $339 \pm 10$ & 328 \\
1621 & $-2.3 \pm 1.8$ & $338 \pm 14$ & 329 \\
1620 & $-0.6 \pm 1.3$ & $325 \pm 10$ & 330 \\
1619 & $-0.3 \pm 1.8$ & $324 \pm 14$ & 331 \\
1618 & $-1.5 \pm 1.7$ & $335 \pm 14$ & 332 \\
1617 & $-4.1 \pm 1.8$ & $357 \pm 15$ & 333 \\
1616 & $-5.4 \pm 1.7$ & $368 \pm 14$ & 334 \\
1615 & $-4.5 \pm 1.3$ & $362 \pm 10$ & 335 \\
1614 & $-1.3 \pm 1.8$ & $337 \pm 15$ & 336 \\
1613 & $-5.2 \pm 1.7$ & $369 \pm 14$ & 337 \\
1612 & $-1.5 \pm 1.8$ & $340 \pm 15$ & 338 \\
1611 & $-6.4 \pm 1.3$ & $381 \pm 11$ & 339 \\
1610 & $-2.7 \pm 1.8$ & $352 \pm 15$ & 340 \\
1609 & $-3.1 \pm 1.6$ & $356 \pm 13$ & 341 \\
1608 & $-3.1 \pm 1.4$ & $357 \pm 12$ & 342 \\
1607 & $-4.8 \pm 2.0$ & $372 \pm 16$ & 343 \\
1606 & $-4.7 \pm 1.1$ & $372 \pm 9$ & 344 \\
1605 & $-5.3 \pm 1.4$ & $378 \pm 11$ & 345 \\
1603 & $-7.2 \pm 1.3$ & $394 \pm 10$ & 346 \\
1602 & $-4.3 \pm 1.9$ & $372 \pm 15$ & 347 \\
& $-2.0 \pm 1.8$ & $354 \pm 15$ & 348
\end{tabular}

TABLE 2. Single-Year Data (Continued)

\begin{tabular}{|c|c|c|c|}
\hline $\mathrm{AD}$ & $\Delta^{14} \mathrm{C}(\% o)$ & ${ }^{14} \mathrm{C}$ BP & \\
\hline 1601 & $-1.5 \pm 1.3$ & $351 \pm 11$ & \\
\hline 1600 & $0.8 \pm 1.2$ & $334 \pm 10$ & \\
\hline 1599 & $-1.0 \pm 1.8$ & $349 \pm 14$ & \\
\hline 1598 & $0.5 \pm 1.7$ & $338 \pm 14$ & \\
\hline 1597 & $-1.5 \pm 1.8$ & $355 \pm 15$ & \\
\hline 1596 & $-0.4 \pm 1.7$ & $347 \pm 14$ & \\
\hline 1595 & $2.2 \pm 1.7$ & $327 \pm 14$ & \\
\hline 1594 & $2.5 \pm 1.8$ & $326 \pm 14$ & \\
\hline 1593 & $0.5 \pm 1.7$ & $343 \pm 13$ & \\
\hline 92 & $-0.3 \pm 2.0$ & $351 \pm 16$ & \\
\hline 91 & $3.7 \pm 1.7$ & $19 \pm 14$ & \\
\hline 1590 & $4.4 \pm 1.8$ & $314 \pm 14$ & \\
\hline 1589 & $3.7 \pm 1.7$ & $321 \pm 14$ & \\
\hline 1588 & $1.1 \pm 1.8$ & $343 \pm 14$ & \\
\hline 1587 & $1.3 \pm 1.7$ & $342 \pm 14$ & \\
\hline 1586 & $2.5 \pm 1.7$ & $334 \pm 14$ & \\
\hline 1585 & $3.7 \pm 1.8$ & $325 \pm 15$ & \\
\hline 1584 & $0.8 \pm 1.8$ & $349 \pm 14$ & \\
\hline 83 & $4.1 \pm 1.7$ & $324 \pm 13$ & 36 \\
\hline 82 & $3.1 \pm 1.7$ & $33 \pm 14$ & \\
\hline & $-0.2 \pm 1.8$ & $361 \pm 14$ & \\
\hline & $3.6 \pm 1.7$ & $31 \pm 14$ & \\
\hline 1579 & $2.5 \pm 1.7$ & $341 \pm 14$ & \\
\hline 1578 & $5.2 \pm 1.7$ & $320 \pm 14$ & 37 \\
\hline 1577 & $3.4 \pm 1.8$ & $336 \pm 15$ & \\
\hline 1576 & $1.8 \pm 1.8$ & $349 \pm 14$ & 37 \\
\hline 1575 & $3.9 \pm 1.7$ & $334 \pm 14$ & \\
\hline & $4.1 \pm 1.8$ & $333 \pm 14$ & \\
\hline & $7.9 \pm 1.9$ & $303 \pm 15$ & \\
\hline & $6.3 \pm 1.8$ & $317 \pm 14$ & \\
\hline & $5.4 \pm 1.9$ & $325 \pm 15$ & \\
\hline & $6.6 \pm 1.8$ & $7 \pm 15$ & \\
\hline & \pm 1 & \pm 14 & \\
\hline & $=1$ & $36 \pm 15$ & \\
\hline & $4.1 \pm 1.3$ & $339 \pm 10$ & \\
\hline & $8.0 \pm 1.2$ & $309 \pm 10$ & \\
\hline & $7.0 \pm 1.9$ & $318 \pm 15$ & \\
\hline & $5.5 \pm 1.9$ & $331 \pm 16$ & \\
\hline & $5.1 \pm 1.8$ & $335 \pm 14$ & 38 \\
\hline & $5.9 \pm 1.7$ & $330 \pm 14$ & \\
\hline & $6.9 \pm 1.8$ & $323 \pm 15$ & \\
\hline & $3.2 \pm 1.8$ & $354 \pm 15$ & 3 \\
\hline & $.6 \pm 1.8$ & $20 \pm 14$ & \\
\hline & $=1.7$ & $3 \pm 14$ & \\
\hline & $7 \pm 1$. & $37 \pm 15$ & \\
\hline & $9.1 \pm 1$ & $10 \pm 15$ & \\
\hline & $9.3 \pm 1.7$ & $309 \pm 13$ & \\
\hline & $9.2 \pm 1$ & $311 \pm 14$ & \\
\hline & & $327 \pm$ & \\
\hline & $13.2 \pm 2.1$ & $281 \pm 16$ & \\
\hline & 9. & $10 \pm 16$ & \\
\hline & $9.4 \pm 1.1$ & $314 \pm 9$ & 40 \\
\hline 154 & $11.6 \pm 2.1$ & $297 \pm 17$ & 40 \\
\hline
\end{tabular}


TABLE 2. Single-Year Data (Continued)

\begin{tabular}{crcr}
\hline cal & & & cal \\
$\mathrm{AD}$ & \multicolumn{1}{c}{$\Delta^{14} \mathrm{C}(\% \circ)$} & ${ }^{14} \mathrm{C} \mathrm{BP}$ & $\mathrm{BP}$ \\
\hline 1548 & $12.1 \pm 1.8$ & $294 \pm 14$ & 402 \\
1547 & $12.6 \pm 1.8$ & $291 \pm 14$ & 403 \\
1546 & $8.8 \pm 1.8$ & $323 \pm 15$ & 404 \\
1545 & $10.9 \pm 1.8$ & $306 \pm 14$ & 405 \\
1544 & $8.6 \pm 1.7$ & $325 \pm 14$ & 406 \\
1543 & $10.2 \pm 1.6$ & $314 \pm 13$ & 407 \\
1542 & $10.0 \pm 1.2$ & $317 \pm 10$ & 408 \\
1541 & $12.1 \pm 1.2$ & $301 \pm 9$ & 409 \\
1540 & $10.0 \pm 1.2$ & $318 \pm 10$ & 410 \\
1539 & $12.5 \pm 1.3$ & $300 \pm 10$ & 411 \\
1538 & $12.3 \pm 1.3$ & $302 \pm 10$ & 412 \\
1537 & $15.6 \pm 1.2$ & $277 \pm 10$ & 413 \\
1536 & $11.4 \pm 1.3$ & $311 \pm 10$ & 414 \\
1535 & $12.4 \pm 1.2$ & $305 \pm 10$ & 415 \\
1534 & $11.1 \pm 1.3$ & $315 \pm 10$ & 416 \\
1533 & $12.3 \pm 1.2$ & $307 \pm 10$ & 417 \\
1532 & $14.5 \pm 1.3$ & $291 \pm 10$ & 418 \\
1531 & $14.3 \pm 1.7$ & $293 \pm 13$ & 419 \\
1530 & $11.5 \pm 1.8$ & $316 \pm 14$ & 420 \\
\hline
\end{tabular}

TABLE 2. Single-Year Data (Continued)

\begin{tabular}{crcr}
\hline cal & & & cal \\
$\mathrm{AD}$ & \multicolumn{1}{c}{$\Delta^{14} \mathrm{C}(\%)$} & \multicolumn{1}{c}{${ }^{14} \mathrm{C} \mathrm{BP}$} & $\mathrm{BP}$ \\
\hline 1529 & $12.8 \pm 1.8$ & $307 \pm 14$ & 421 \\
1528 & $12.6 \pm 1.6$ & $310 \pm 13$ & 422 \\
1527 & $11.5 \pm 1.8$ & $319 \pm 14$ & 423 \\
1526 & $12.4 \pm 1.8$ & $313 \pm 14$ & 424 \\
1525 & $10.6 \pm 1.3$ & $328 \pm 10$ & 425 \\
1524 & $10.0 \pm 1.8$ & $334 \pm 14$ & 426 \\
1523 & $10.2 \pm 1.7$ & $334 \pm 14$ & 427 \\
1522 & $9.1 \pm 2.0$ & $344 \pm 16$ & 428 \\
1521 & $11.0 \pm 2.0$ & $329 \pm 16$ & 429 \\
1520 & $8.5 \pm 1.8$ & $350 \pm 14$ & 430 \\
1519 & $6.4 \pm 1.9$ & $367 \pm 16$ & 431 \\
1517 & $11.6 \pm 1.8$ & $328 \pm 14$ & 433 \\
1516 & $9.2 \pm 1.7$ & $348 \pm 14$ & 434 \\
1515 & $8.5 \pm 1.6$ & $355 \pm 13$ & 435 \\
1514 & $10.0 \pm 1.8$ & $344 \pm 14$ & 436 \\
1513 & $10.5 \pm 1.8$ & $341 \pm 14$ & 437 \\
1512 & $10.0 \pm 1.8$ & $346 \pm 14$ & 438 \\
1511 & $6.9 \pm 1.7$ & $371 \pm 13$ & 439 \\
1510 & $8.5 \pm 1.8$ & $359 \pm 14$ & 440 \\
\hline
\end{tabular}

